\title{
ACTUALIZACIÓN A LA LISTA DE COLEÓPTEROS (INSECTA: COLEOPTERA) DE LA REGIÓN PIURA, PERÚ
}

\author{
Gino Juárez-Noé1,* \& Uzbekia González-Coronado² \\ ${ }^{1}$ Distrito de Piura, Región Piura-Perú. e-mail: norbiol@hotmail.com \\ ORCID iD: https://orcid.org/0000-0002-8847-6059 \\ ${ }^{2}$ Distrito de Piura, Región Piura-Perú. e-mail: issa.gonzalez06@gmail.com \\ ORCID iD: https://orcid.org/0000-0001-5920-7549 \\ *Autor para correspondencia: Gino Juárez-Noé E-mail: orbiol@hotmail.com
}

\section{RESUMEN}

Con base en colectas, registros en la bibliografía y revisión de material depositado en colecciones entomológicas se realiza una actualización a la lista de coleópteros de la región Piura en Perú, añadiendo nuevas especies y nuevos datos distribucionales. Se adicionan 72 especies, 52 géneros y 5 familias, con lo cual la cantidad de coleópteros para la región se eleva a 317 especies, 255 géneros y 38 familias, mientras que para 13 especies ya conocidas se reportan nuevos datos provinciales ampliando su distribución dentro de la región. Ahasverus advena (Waltl, 1834), Alaetrinus pullus (Sahlberg, 1823), Taeniotes marmoratus Thomson, 1865 y el género Coscinoptera Lacordaire, 1848 se citan por primera vez para Perú, mientras que Pagiocerus frontalis (Fabricius, 1801), Scymnobius ecuadoricus Gordon \& González, 2005 y Rodolia cardinalis (Mulsant, 1850) son nuevas citas para la región Piura. Las especies consideradas en esta actualización se presentan a modo de listado faunístico comentado y se incluye lista actualizada de todos los coleópteros conocidos hasta el momento para la región con distribución por provincia y paisaje ecológico.

Palabras clave: Coleoptera; actualización; distribución; paisaje ecológico; región Piura; Perú.

\section{ABSTRACT}

Update to the checklist of beetles (Insecta: Coleoptera) of Piura region, Peru

An update of the checklist of beetles of the Piura region in Peru is made based on specimen collecting, bibliographic records and a review of the material deposited in entomological collections, adding new species and new distributional data. 72 species, 52 genera and 5 families are added, with which the number of beetles for the region rises to 317 species, 255 genera and 38 families, while for 13 already known species, new provincial data are reported expanding its distribution within the region. Ahasverus advena (Waltl, 1834), Alaetrinus pullus (Sahlberg, 1823), Taeniotes marmoratus Thomson, 1865 and genus Coscinoptera Lacordaire, 1848 are cited for the first time for Peru, while Pagiocerus frontalis (Fabricius, 1801), Scymnobius ecuadoricus Gordon \& González, 2005 and Rodolia cardinalis (Mulsant, 1850) are new records for the Piura region. The species considered in this update are presented as a commented faunistic list and an update list of all the beetles known until now for the region with distribution by province and ecological landscape is included.

Keywords: Coleoptera; update; distribution; landscape ecological; Piura region; Peru.

Recibido/Received: 15/01/2019; Aceptado/Accepted: 26/07/2019; Publicado en línea/Published online: 23/09/2019

Cómo citar este artículo/Citation: Juárez-Noé, G. \& González-Coronado, U. 2019. Actualización a la lista de coleópteros (Insecta: Coleoptera) de la región Piura, Perú. Graellsia, 75(2): e097. https://doi.org/10.3989/graellsia.2019.v75.233

Copyright: (C) 2019 SAM \& CSIC. This is an open-access article distributed under the terms of the Creative Commons Attribution 4.0 International (CC BY 4.0) License. 


\section{Introducción}

El orden Coleoptera en Perú está representado por aproximadamente 10,000 especies descritas y 99 familias, siendo uno de los grupos de insectos más diversos del país (Chaboo, 2015). Los coleópteros por su gran abundancia, diversidad ecológica y por estar asociados con formaciones vegetales donde actúan como depredadores, herbívoros, polinizadores y descomponedores de materia orgánica, cobran especial importancia en estudios ecológicos, especialmente como indicadores de biodiversidad y conservación (Lozada et al., 2004).

La gran variedad fisiográfica y climática que caracteriza a la región Piura, ubicada al extremo noroeste de Perú, han determinado la existencia de zonas geomorfológicas y ecológicas importantes, originando una gran heterogeneidad de ecosistemas y variados gradientes altitudinales, lo que ha dado lugar a una notable riqueza vegetacional y faunística con altos niveles de endemismos (More et al., 2014).

En una reciente publicación, Juárez-Noé \& González-Coronado (2018) con base en colectas y registros en la literatura elaboraron una lista de coleópteros para la región Piura en la que registraron 246 especies, 203 géneros y 33 familias, incluyendo datos distribucionales, siendo hasta el momento la única lista que detalla la cantidad y distribución de los coleópteros presentes para esa región. Siguiendo ese procedimiento, se actualiza ese listado con el registro de 72 especies, 52 géneros y 5 familias, la cantidad y distribución de coleópteros de la región.

\section{Material y métodos}

Todas las especies consideradas en esta actualización se basan en colectas de especímenes, registros de coleópteros en la bibliografía y revisión de material depositado en colecciones entomológicas.

El trabajo de campo fue realizado por los autores del manuscrito a través de colectas en 26 localidades de muestreo que abarcaron las 8 provincias de la región y un rango altitudinal desde el nivel del mar hasta los 3,100 msnm (Apéndice 1); las colectas tuvieron una duración de 3 días por localidad y se llevaron a cabo mensualmente desde enero 2018 a marzo 2019. En cada localidad de muestreo se utilizaron trampas pitfall, formadas por vasos plásticos transparentes de $500 \mathrm{ml} \mathrm{y} 9 \mathrm{~cm}$ de diámetro de abertura llenados hasta su mitad con una mezcla de siete partes de agua por una de formol al $40 \%$ y un poco de detergente y cebadas con heces de ganado vacuno y frutas fermentadas de plátano mezcladas con cerveza, vainilla, azúcar (Giraldo \& Arellano, 2003) e instaladas en un transecto lineal de $100 \mathrm{~m}$ con 10 trampas separadas cada $10 \mathrm{~m}$ y revisadas cada 12 horas (Villarreal et al., 2004); dos trampas luz formadas por dos focos de 100 watts cada uno de luz blanca colocadas frente y debajo de mantas blancas de dos metros de ancho y dos metros de largo las cuales estuvieron activas entre las 19:00 h y 23:00 h (Solís, 2004); una red acuática de $30 \mathrm{~cm}$ de diámetro y $40 \mathrm{~cm}$ de fondo, con una malla de $0,1 \mathrm{~mm}$ (Benetti et al., 2003) y se realizó recolecta directa, tanto diurna como nocturna, revisando el follaje de las plantas, flores, hojarasca, troncos podridos, bajo piedras, animales en descomposición y excremento (Morón, 2004).

Los registros de coleópteros a través de bibliografía provienen de las publicadas en revistas científicas, tesis de maestría y doctorado, quedando excluidos los informes técnicos o de impacto ambiental y resúmenes de congresos cuyos datos generalmente no han sido revisados por pares. Los trabajos consultados fueron los de Gidaspow (1963), Howden (1985), Ahn \& Ashe (1996), Jameson (1997), Casari (2002), Soula (2002), Arnaud \& Bomans (2006), Constantin (2009, 2011), Asiain et al. (2010), Rocha (2010), CoelhoGrossi (2011), Chani-Posse \& Asenjo (2013), Arriagada (2015), Ratcliffe (2015), Puthz (2017), Moore et al. (2018), Nilsson \& Hájek (2018), Opitz (2018), Salazar \& Boucher (2018), Rossini et al. (2018), Komiya \& Santos-Silva (2018), GiraldoMendoza (2019) y Moore \& Diéguez (2019). Asimismo con base en revisión de material de la colección Gino Juárez Noé (CGJN) Piura-Perú, donde se encuentran depositados los ejemplares de las especies citadas en la lista inicial de coleópteros para la región Piura, se completó la identificación de 10 géneros que estaban consideradas como morfoespecies en dicha lista (Juárez-Noé \& González-Coronado, 2018).

Para la identificación de los especímenes colectados y de los ejemplares depositados en la colección entomológica se utilizaron los trabajos de Barber (1936), Dillon \& Dillon (1941), Blake (1950), Kulzer (1958), Breuning (1966), Martins (1975), Ratcliffe (1976), Noonan (1981), Vaurie (1982), Lachaume (1985), Gorham (1991), Iwan (1995), Valle da Silva \& Massutti de Almeida (2001), Gordon \& González (2005), Smith (2003), Rees (2004), Ciro-Díaz et al. (2008), Soula (2008), Bustamante-Navarrete et al. (2017), Kingsolver et al. (2017), Ratcliffe (2018), Giraldo-Mendoza \& Flores (2019) y López-García et al. (2019). Todos los ejemplares estudiados en esta actualización fueron depositados en la colección del Museo de Entomología de la Universidad Nacional de Tumbes, Perú (MEUNT).

Todas las especies presentadas están ordenadas siguiendo la clasificación de Bouchard et al. (2011), sin embargo, dentro de Chrysomelidae se considera a Alticinae como subfamilia (Furth 
et al., 2015). Las especies consideradas en esta actualización se presentan a modo de listado faunístico comentado, siendo la secuencia del material estudiado la siguiente: número de $\widehat{\partial} \widehat{\partial}$, $ᄋ$, provincia, distrito, localidad, coordenadas geográficas, altitud en msnm, fecha de captura, tipo de captura, recolector, tipo de paisaje ecológico, código de registro cuando procedió de material depositado en la colección GJN y de una referencia para indicar los autores de los trabajos revisados cuando se recopiló a través de bibliografía. Las nuevas especies añadidas para la región se indican con una cruz $(\dagger)$, mientras que las especies conocidas únicamente de Perú (Casari, 2002; Arnaud \& Bomans, 2006; González, 2015; Ratcliffe et al., 2015; Smith et al., 2015; Chandler, 2016; Giraldo \& Flores, 2016; Pinto \& Bologna, 2016; Salazar \& Boucher, 2018; Komiya \& Santos-Silva, 2018; Ratcliffe, 2018; Giraldo-Mendoza, 2019; Moore \& Diéguez, 2019) se indican con un asterisco (*). Asimismo, se aporta una lista actualizada de todos los coleópteros conocidos hasta el momento para la región Piura incluyendo para cada especie su distribución por provincia y paisaje ecológico. La clasificación y abreviaciones para cada provincia y paisaje ecológico se asignaron siguiendo los criterios propuestos por Juárez-Noé \& GonzálezCoronado (2018).

\section{Resultados y discusión}

En total han sido consideradas 95 especies para esta actualización, de las cuales 52 provienen de recolectas (de un total de 1,023 ejemplares recuperados con los métodos de colecta utilizados), 33 de registros bibliográficos y 10 de revisión de material depositado en la colección GJN. De las provenientes a través de recolectas y citas bibliográficas 72 especies, 52 géneros y 5 familias no se registran en la lista inicial de coleópteros para la región Piura (Juárez-Noé \& González-Coronado, 2018), mientras que las 10 especies del material depositado provienen de la revisión de especímenes de los géneros Notiobia Perty, 1830, Platycoelia Dejean, 1833, Tomarus Erichson, 1847, Smodicum Haldeman, 1847, Taeniotes Audinet-Serville, 1835, Bisaltes Thomson, 1860, Parapraocis Casey, 1907, Philorea Erichson, 1834, Prohylitus Kaszab, 1964 y Myochrous Dejean, 1837, los mismos que en la lista inicial estaban citados como morfoespecies. En la lista inicial de coleópteros para la región Piura (Juárez-Noé \& González-Coronado, 2018) se registraron 33 familias, 203 géneros y 246 especies, de los cuales 160 se citan netamente como especies y 86 como morfoespecies. Es importante mencionar que de esta lista se está excluyendo a Neda norrisi (Guerin-Meneville, 1842), ya que según González
(2007) podría tratarse de una especie diferente. Por lo tanto, en el presente trabajo, con la adición de las 72 especies y 52 géneros provenientes de recolectas y registros bibliográficos y de las 10 especies de la revisión de material depositado, la nueva lista de coleópteros para la región Piura queda conformada por 38 familias, 255 géneros y 317 especies, de las cuales 237 se citan netamente como especies y 80 como morfoespecies (Tabla 1).

Esta nueva lista de coleópteros incluye a Ahasverus advena (Walt1, 1834), Alaetrinus pullus (Sahlberg, 1823), Taeniotes marmoratus Thomson, 1865 y al género Coscinoptera Lacordaire, 1848 como nuevos registros para Perú, Pagiocerus frontalis (Fabricius, 1801), Scymnobius ecuadoricus Gordon \& González, 2005 y Rodolia cardinalis (Mulsant, 1850) como nuevos registros para la región Piura, así como a 28 especies conocidas únicamente de Perú (Tabla 1). Las 5 familias añadidas son Gyrinidae, Silphidae, Mordellidae, Oedemeridae y Anthribidae, mientras que las 38 familias registradas representan ahora el $38 \%$ de las citadas a nivel nacional (Chaboo, 2015), siendo Scarabaeidae con 48, Coccinellidae con 36, Chrysomelidae con 35, Tenebrionidae con 23 y Cerambycidae con 21 las que presentan mayor cantidad de especies (Tabla 1).

Del mismo modo, para 13 especies provenientes de colectas y que se encuentran citadas en la lista inicial de coleópteros (Juárez-Noé \& GonzálezCoronado, 2018), se añaden nuevos datos provinciales ampliando su distribución dentro de la región. Así, Aphodius (Labarrus) pseudolividus Balthasar, 1941 y Cissites maculata (Swederus, 1787) se distribuyen ahora en todas las provincias y paisajes ecológicos, mientras que Gymnetis merops Ratcliffe, 2018, Sinoxylon anale Lesne, 1897, Azya orbigera ecuadorica Gordon, 1980, Pentilia sp, Brachiacantha darlene Gordon \& Canepari, 2014, Coleomegilla maculata bisexpunctata Latreille, 1833, Harmonia axyridis (Pallas, 1772), Heterachthes pallidipennis (Thomson, 1865), Rhynchophorus palmarum Linnaeus, 1758 y Eustylus humilis (Erichson, 1847) se distribuyen ahora en más de una provincia pero muestran distribución concordante con el paisaje ecológico con el cual estaban citados en la lista inicial, que corresponde a bosques estacionalmente secos. Esto también sucede para algunas especies recién añadidas como Paederus ornaticornis Sharp, 1891, Meridiorhantus calidus (Fabricius, 1792) y Alphitobius diaperinus (Panzer, 1797) que se distribuyen en todas las provincias y paisajes ecológicos y para Alaetrinus pullus (Sahlberg, 1823) y Tomarus peruvianus (Endrödi, 1970) cuya distribución abarca todas las provincias pero que ecológicamente se limitan, por el momento, a los bosques estacionalmente secos. 
Tabla 1.- Lista actualizada de los coleópteros presentes en la región Piura, Perú. Se incluye distribución por provincias (PIU: Piura; SULL: Sullana; TAL: Talara; PAI: Paita; SECH: Sechura; MORR: Morropón; HUA: Huancabamba; AYA: Ayabaca) y paisaje ecológico (BESIl: Bosque estacionalmente seco de llanura; BESc: Bosque estacionalmente seco de colina; BESm: Bosque estacionalmente seco de montaña; BSI: Bosque seco interandino; DES: Desierto; MAN: Manglar; BHM: Bosque húmedo de montaña). Los asteriscos representan especies conocidas únicamente de Perú.

Table 1.- Updated checklist of beetles present in the Piura region, Peru. It includes distribution by provinces (PIU: Piura; SULL: Sullana; TAL: Talara; PAI: Paita; SECH: Sechura; MORR: Morropón; HUA: Huancabamba; AYA: Ayabaca) and ecological landscape (BESII: Seasonally dry plain forest; BESc: Seasonally dry hill forest; BESm: Seasonally dry mountain forest; BSI: Dry inter-Andean forest; DES: Desert; MAN: mangrove swamp; BHM: mountain rainforest). Asterisks represent species known only from Peru.

\begin{tabular}{|c|c|c|}
\hline Taxones & Distribución (provincia) (paisaje ecológico) & Referencia \\
\hline \multicolumn{3}{|l|}{ SUBORDEN ADEPHAGA } \\
\hline \multicolumn{3}{|l|}{ Gyrinidae } \\
\hline \multicolumn{3}{|l|}{ Gyrininae Latreille, 1810} \\
\hline \multicolumn{3}{|l|}{ Andogyrus Ochs, 1924} \\
\hline 1. Andogyrus peruvianus Régimbart, $1907 \dagger$ & (AYA) (BHM) & \\
\hline \multicolumn{3}{|l|}{ Carabidae } \\
\hline \multicolumn{3}{|l|}{ Cicindelinae Latreille, 1802} \\
\hline \multicolumn{3}{|l|}{ Cicindelidia Linne, 1758} \\
\hline 2. Cicindelidia trifasciata Fabricius, 1871 & $\begin{array}{l}\text { (PIU, SULL, PAI, TAL, SECH, MORR) (BESII, BESc, } \\
\text { DES, MAN) }\end{array}$ & Juárez-Noé \& González-Coronado (2018) \\
\hline \multicolumn{3}{|l|}{ Tetracha Hope, 1838} \\
\hline $\begin{array}{l}\text { 3. Tetracha chilensis (Laporte de Castelnau, } \\
\text { 1834) }\end{array}$ & $\begin{array}{l}\text { (PIU, SULL, PAI, TAL, SECH, MORR) (BESII, DES, } \\
\text { MAN) }\end{array}$ & Juárez-Noé \& González-Coronado (2018) \\
\hline 4. Tetracha suturalis W. Horn, 1900 & $(\mathrm{SECH})(\mathrm{DES}, \mathrm{MAN})$ & Juárez-Noé \& González-Coronado (2018) \\
\hline \multicolumn{3}{|l|}{ Carabinae } \\
\hline \multicolumn{3}{|l|}{ Calosoma Weber, 1801} \\
\hline $\begin{array}{l}\text { 5. Calosoma (Castrida) abbreviatum Chaudoir, } \\
1869\end{array}$ & $\begin{array}{l}\text { (PIU, SULL, PAI, TAL, SECH, MORR) (BESII, BESc, } \\
\text { BESm, DES, MAN) }\end{array}$ & Juárez-Noé \& González-Coronado (2018) \\
\hline 6. Calosoma (Castrida) rufipenne Dejean, $1831 \dagger$ & $(\mathrm{AYA})(\mathrm{BESc})$ & Gidaspow (1963) \\
\hline \multicolumn{3}{|l|}{ Harpalinae Bonelli, 1810} \\
\hline \multicolumn{3}{|l|}{ Notiobia Perty, 1830} \\
\hline $\begin{array}{l}\text { 7. Notiobia (Anisotarsus) peruviana (Dejean, } \\
\text { 1829) }\end{array}$ & (PIU, SULL, TAL, SECH, PAI, MORR) (BESII, DES) & Juárez-Noé \& González-Coronado (2018) \\
\hline \multicolumn{3}{|l|}{ Blennidus Motschulsky, 1865} \\
\hline 8. Blennidus peruvianus (Dejean, 1828) & (PIU, SULL, TAL, SECH, PAI, MORR) (BESII, DES) & Juárez-Noé \& González-Coronado (2018) \\
\hline 9. Blennidus sp. 1. & (PIU) (BESII) & Juárez-Noé \& González-Coronado (2018) \\
\hline \multicolumn{3}{|l|}{ Calleida Latreille \& Dejean, 1824} \\
\hline 10. Calleida migratoria Casale, 2002 & (PIU) (BESII) & Juárez-Noé \& González-Coronado (2018) \\
\hline 11. Calleida sp. 1. & (PIU) (BESII) & Juárez-Noé \& González-Coronado (2018) \\
\hline \multicolumn{3}{|l|}{ Stenolophus Dejean, 1821} \\
\hline 12. Stenolophus sp. 1 & (PIU) (BESII) & Juárez-Noé \& González-Coronado (2018) \\
\hline \multicolumn{3}{|l|}{ Tetragonoderus Dejean, 1821} \\
\hline 13. Tetragonoderus sp. 1. & (PIU) (BESII) & Juárez-Noé \& González-Coronado (2018) \\
\hline \multicolumn{3}{|l|}{ Selenophorus Dejean, 1821} \\
\hline 14. Selenophorus sp.1. & (PIU) (BESII) & Juárez-Noé \& González-Coronado (2018) \\
\hline \multicolumn{3}{|l|}{ Brachininae Bonelli, 1810} \\
\hline \multicolumn{3}{|l|}{ Brachinus Weber, 1801} \\
\hline 15. Brachinus sp. 1. & (PIU) (BESII) & Juárez-Noé \& González-Coronado (2018) \\
\hline \multicolumn{3}{|l|}{ Scaratinae Bonelli, 1810} \\
\hline \multicolumn{3}{|l|}{ Clivina Weber, 1801} \\
\hline 16. Clivina (Paraclivina) sp. 1. & (PIU, SULL, PAI, TAL) (BESII, BESc, BESm) & Juárez-Noé \& González-Coronado (2018) \\
\hline \multicolumn{3}{|l|}{ Dytiscidae Leach, 1815} \\
\hline \multicolumn{3}{|l|}{ Dytiscinae Leach, 1815} \\
\hline Megadytes Sharp, 1882 & & \\
\hline
\end{tabular}


Tabla 1 (continuación).

\begin{tabular}{|c|c|c|}
\hline Taxones & Distribución (provincia) (paisaje ecológico) & Referencia \\
\hline $\begin{array}{l}\text { 17. Megadytes (Bifurcitus) Ihermineri (Guérin- } \\
\text { Méneville, 1829) }\end{array}$ & $\begin{array}{l}\text { (PIU, SULL, TAL, PAI, SECH, MORR, HUA, AYA) } \\
\text { (BESII, BESC, BESm, BSI, DES, MAN, BHM) }\end{array}$ & Juárez-Noé \& González-Coronado (2018) \\
\hline $\begin{array}{l}\text { 18. Megadytes (Megadytes) laevigatus (Olivier, } \\
\text { 1795) }\end{array}$ & $\begin{array}{l}\text { (PIU, SULL, TAL, PAI, SECH, MORR, HUA, AYA) } \\
\text { (BESII, BESc, BESm, BSI, DES, MAN, BHM) }\end{array}$ & Juárez-Noé \& González-Coronado (2018) \\
\hline \multicolumn{3}{|l|}{ Thermonectus Dejean, 1837} \\
\hline 19. Thermonectus succinctus (Aubé, 1838). & $\begin{array}{l}\text { (PIU, SULL, TAL, PAI, SECH, MORR, HUA, AYA) } \\
\text { (BESII, BESc, BESm, BSI, MAN, DES, BHM) }\end{array}$ & \\
\hline \multicolumn{3}{|l|}{ Agabinae Thomson, 1867} \\
\hline \multicolumn{3}{|l|}{ Agametrus Sharp, 1882} \\
\hline 20. Agametrus monticola (Guignot, 1958)† & (AYA) (BHM) & Nilsson \& Hájek (2018) \\
\hline 21. Agametrus andinus Guignot, $1958 \dagger$ & (AYA) (BHM) & Nilsson \& Hájek (2018) \\
\hline \multicolumn{3}{|l|}{ Copelatinae Branden, 1885} \\
\hline \multicolumn{3}{|l|}{ Copelatus Erichson, 1832} \\
\hline 22. Copelatus blancasi Guignot, $1958 \dagger$ & (AYA) (BHM) & Nilsson \& Hájek (2018) \\
\hline \multicolumn{3}{|l|}{ Colymbetinae Erichson, 1837} \\
\hline \multicolumn{3}{|l|}{ Meridiorhantus Balke, Hájek \& Hendrich, 2017} \\
\hline 23. Meridiorhantus calidus (Fabricius, 1792)† & $\begin{array}{l}\text { (PIU, SULL, TAL, PAI, SECH, MORR, HUA, AYA) } \\
\text { (BESII, DES, MAN, BSI, BHM) }\end{array}$ & \\
\hline \multicolumn{3}{|l|}{ Laccophilinae Gistel, 1856} \\
\hline \multicolumn{3}{|l|}{ Laccophilus Leach, 1815} \\
\hline 24. Laccophilus normifer Guignot, $1958 \dagger$ & (AYA) (BHM) & Nilsson \& Hájek (2018) \\
\hline \multicolumn{3}{|l|}{ SUBORDEN POLYPHAGA } \\
\hline \multicolumn{3}{|l|}{ Hydrophilidae Latreille, 1802} \\
\hline \multicolumn{3}{|l|}{ Hydrophilinae Latreille, 1802} \\
\hline \multicolumn{3}{|l|}{ Hydrophilus Geoffroy, 1762} \\
\hline $\begin{array}{l}\text { 25. Hydrophilus (Hydrophilus) foveolatus } \\
\text { (Régimbart, 1901) }\end{array}$ & $\begin{array}{l}\text { (PIU, SULL, TAL, PAI, SECH, MORR, HUA, AYA) } \\
\text { (BESII, BESc, BESm, BSI, MAN, DES, BHM) }\end{array}$ & Juárez-Noé \& González-Coronado (2018) \\
\hline \multicolumn{3}{|l|}{ Tropisternus Solier, 1834} \\
\hline $\begin{array}{l}\text { 26. Tropisternus (Strepitornus) collaris (Fabricius, } \\
\text { 1775) }\end{array}$ & $\begin{array}{l}\text { (PIU, SULL, TAL, PAI, SECH, MORR, HUA, AYA) } \\
\text { (BESII, BESc, BESm, BSI, MAN, DES, BHM) }\end{array}$ & Juárez-Noé \& González-Coronado (2018) \\
\hline 27. Tropisternus sp. 1. & $\begin{array}{l}\text { (PIU, SULL, TAL, PAI, SECH, MORR, HUA, AYA) } \\
\text { (BESII, BESc, BESm, BSI, MAN, DES, BHM) }\end{array}$ & Juárez-Noé \& González-Coronado (2018) \\
\hline \multicolumn{3}{|l|}{ Histeridae Gyllenhal, 1808} \\
\hline \multicolumn{3}{|l|}{ Histerinae Gyllenhal, 1808} \\
\hline \multicolumn{3}{|l|}{ Omalodes Dejean, 1833} \\
\hline 28. Omalodes sp. 1 & (MORR) (BESc) & Juárez-Noé \& González-Coronado (2018) \\
\hline \multicolumn{3}{|l|}{ Saprinae Blanchard, 1845} \\
\hline \multicolumn{3}{|l|}{ Euspilotus Lewis, 1907} \\
\hline $\begin{array}{l}\text { 29. Euspilotus (Hesperosaprinus) erythropleurus } \\
\text { (Marseul, 1855)† }\end{array}$ & (SULL) (BESII) & Arriagada (2015) \\
\hline \multicolumn{3}{|l|}{ Hypocaccus Thomson, 1867} \\
\hline $\begin{array}{l}\text { 30. Hypocaccus (Baeckmanniolus) gaudens } \\
\text { (LeConte, 1851)† }\end{array}$ & (SULL, TAL) (BESII, DES) & Arriagada (2015) \\
\hline \multicolumn{3}{|l|}{ Silphidae Latreille, 1806} \\
\hline \multicolumn{3}{|l|}{ Silphinae Latreille, 1806} \\
\hline \multicolumn{3}{|l|}{ Oxelytrum Gistel, 1848} \\
\hline 31. Oxelytrum anticola (Guérin-Méneville, 1855)† & (HUA) (BHM) & \\
\hline Staphylinidae Latreille, 1802 & & \\
\hline Oxytelinae Fleming, 1821 & & \\
\hline Bledius Leach, 1819 & & \\
\hline 32. Bledius sp. 1. & $\begin{array}{l}\text { (PIU, TAL, SULL, PAI, SECH, MORR) (BESII, BESc, } \\
\text { MAN, DES) }\end{array}$ & Juárez-Noé \& González-Coronado (2018) \\
\hline Paederinae Fleming, 1821 & & \\
\hline Eupaederus Fabricius, 1775 & & \\
\hline
\end{tabular}


Tabla 1 (continuación).

\begin{tabular}{|c|c|c|}
\hline Taxones & Distribución (provincia) (paisaje ecológico) & Referencia \\
\hline $\begin{array}{l}\text { 33. Eupaederus cyanipennis (Guérin-Méneville, } \\
\text { 1830) }\end{array}$ & $\begin{array}{l}\text { (PIU, SULL, TAL, PAI, SECH, MORR) (BESII, BESc, } \\
\text { DES) }\end{array}$ & Juárez-Noé \& González-Coronado (2018) \\
\hline \multicolumn{3}{|l|}{ Paederus Fabricius, 1775} \\
\hline 34. Paederus ornaticornis Sharp, 1891† & $\begin{array}{l}\text { (PIU, SULL, TAL, PAI, SECH, MORR, HUA, AYA) } \\
\text { (BESII, BESc, BESm DES, MAN, BSI, BHM) }\end{array}$ & \\
\hline \multicolumn{3}{|l|}{ Medon Stephens, 1833} \\
\hline 35. Medon sp. 1. & (PIU) (BESII) & Juárez-Noé \& González-Coronado (2018) \\
\hline \multicolumn{3}{|l|}{ Staphylininae Latreille, 1802} \\
\hline \multicolumn{3}{|l|}{ Philonthus Stephens, 1829} \\
\hline 36. Philonthus sp. 1 & (PIU, SULL, TAL, PAI, SECH) (BESII, DES) & Juárez-Noé \& González-Coronado (2018) \\
\hline \multicolumn{3}{|l|}{ Leptopeltus Bernhauer, 1906} \\
\hline 37. Leptopeltus flavipennis (Erichson, 1840)† & $(\mathrm{AYA})(\mathrm{BHM})$ & Chani-Posse \& Asenjo (2013) \\
\hline \multicolumn{3}{|l|}{ Plochionocerus Dejean, 1833} \\
\hline 38. Plochionocerus humeralis (Sharp, 1885)† & (SULL) (BESc) & Asiain et al., (2010) \\
\hline \multicolumn{3}{|l|}{ Steninae MacLeay, 1825} \\
\hline \multicolumn{3}{|l|}{ Stenus Latreille, 1796} \\
\hline 39. Stenus subcoeruleus Bernhauer, 1916† & $(\mathrm{HUA})(\mathrm{BHM})$ & Puthz (2017) \\
\hline \multicolumn{3}{|l|}{ Aleocharinae Fleming, 1821} \\
\hline \multicolumn{3}{|l|}{ Rothium Moore \& Legner, 1977} \\
\hline 40. Rothium evansi Ahn \& Ashe, 1996† & (PIU, PAI) (BESII) & Ahn \& Ashe (1996) \\
\hline \multicolumn{3}{|l|}{ Aleochara Gravenhorst, 1802} \\
\hline 41. Aleochara sp $\dagger$ & (PAI) (DES) & \\
\hline \multicolumn{3}{|l|}{ Geotrupidae Latreille, 1802} \\
\hline \multicolumn{3}{|l|}{ Bolboceratinae Mulsant, 1842} \\
\hline \multicolumn{3}{|l|}{ Zefevazia Martínez, 1954} \\
\hline 42. Zefevazia peruana (Boucomont, 1902)* & (PIU) (BESII) & Juárez-Noé \& González-Coronado (2018) \\
\hline \multicolumn{3}{|l|}{ Neoathyreus Howden \& Martinez, 1963} \\
\hline $\begin{array}{l}\text { 43. Neoathyreus (Neoathyreus) perryae Howden, } \\
\text { 1985† }\end{array}$ & $(\mathrm{TAL})(\mathrm{BESII})$ & Howden (1985) \\
\hline \multicolumn{3}{|l|}{ Passalidae Leach, 1815} \\
\hline \multicolumn{3}{|l|}{ Passalinae Leach, 1815} \\
\hline \multicolumn{3}{|l|}{ Passalus Fabricius, 1792} \\
\hline 44. Passalus sp. 1. & (HUA, AYA) (BSI, BHM) & Juárez-Noé \& González-Coronado (2018) \\
\hline \multicolumn{3}{|l|}{ Veturius (Kaup, 1871) } \\
\hline 45. Veturius (Veturius) standfussi Kuwert, 1891† & $(\mathrm{HUA})(\mathrm{BHM})$ & Salazar \& Boucher (2018) \\
\hline $\begin{array}{l}\text { 46. Veturius (Ouayana) quaesitor Boucher \& } \\
\text { Salazar, } 2018^{*} \dagger\end{array}$ & (AYA) (BESc) & Salazar \& Boucher (2018) \\
\hline \multicolumn{3}{|l|}{ Trogidae MacLeay, 1819} \\
\hline \multicolumn{3}{|l|}{ Omorginae Nikolajev, 2005} \\
\hline \multicolumn{3}{|l|}{ Omorgus Erichson, 1847} \\
\hline $\begin{array}{l}\text { 47. Omorgus (Omorgus) persuberosus (Vaurie, } \\
\text { 1962) }\end{array}$ & $\begin{array}{l}\text { (PIU, SULL, TAL, PAI, SECH, MORR) (BESII, BESc, } \\
\text { DES) }\end{array}$ & Juárez-Noé \& González-Coronado (2018) \\
\hline \multicolumn{3}{|l|}{ Lucanidae Latreille, 1804} \\
\hline Syndesinae MacLeay, 1819 & & \\
\hline Psilodon Perty, 1830 & & \\
\hline 48. Psilodon sp. 1 & (AYA) (BHM) & Juárez-Noé \& González-Coronado (2018) \\
\hline Lucaninae Latreille, 1804 & & \\
\hline Sphaenognathus Buquet, 1838 & & \\
\hline $\begin{array}{l}\text { 49. Sphaenognathus (Chiasognathinus) } \\
\text { xerophilus Bartolozzi \& Onore, } 2006\end{array}$ & (HUA) (BHM) & Juárez-Noé \& González-Coronado (2018) \\
\hline Incadorcus Arnaud \& Bomans, 2006 & & \\
\hline $\begin{array}{l}\text { 50. Incadorcus damasoi Arnaud \& Bomans } \\
2006^{*} \dagger\end{array}$ & (AYA) (BESm) & Arnaud \& Bomans (2006) \\
\hline Aegognathus Leuthner, 1883 & & \\
\hline
\end{tabular}


Tabla 1 (continuación).

\begin{tabular}{|c|c|c|}
\hline Taxones & Distribución (provincia) (paisaje ecológico) & Referencia \\
\hline $\begin{array}{l}\text { 51. Aegognathus leuthneri damasoi Arnaud \& } \\
\text { Bomans } 2006^{*} \dagger\end{array}$ & $(\mathrm{AYA})(\mathrm{BESm})$ & Arnaud \& Bomans (2006) \\
\hline \multicolumn{3}{|l|}{ Sclerostomus Burmeister, 1847} \\
\hline $\begin{array}{l}\text { 52. Sclerostomus damasoi Arnaud \& Bomans } \\
2006^{*} \dagger\end{array}$ & $(\mathrm{AYA})(\mathrm{BESm})$ & Arnaud \& Bomans (2006) \\
\hline $\begin{array}{l}\text { 53. Sclerostomus wendyae Arnaud \& Bomans } \\
2006^{*} \dagger\end{array}$ & (AYA) (BESm) & Coelho-Grossi (2011) \\
\hline \multicolumn{3}{|l|}{ Scarabaeidae Latreille, 1802} \\
\hline \multicolumn{3}{|l|}{ Scarabaeinae Latreille, 1802} \\
\hline \multicolumn{3}{|l|}{ Cryptocanthon Balthasar, 1942} \\
\hline 54. Cryptocanthon paradoxus Balthasar, 1942 & (AYA) (BHM) & Juárez-Noé \& González-Coronado (2018) \\
\hline \multicolumn{3}{|l|}{ Onthophagus Latreille, 1802} \\
\hline 55. Onthophagus confusus Boucomont, 1932† & (MORR) (BESc) & Rossini et al., (2018) \\
\hline \multicolumn{3}{|l|}{ Uroxys Westwood, 1842} \\
\hline 56. Uroxys sp. 1 & (AYA) (BHM) & Juárez-Noé \& González-Coronado (2018) \\
\hline \multicolumn{3}{|l|}{ Dichotomius Hope, 1838} \\
\hline 57. Dichotomius cotopaxi (Guerin, 1855) & (AYA) (BHM) & Juárez-Noé \& González-Coronado (2018) \\
\hline \multicolumn{3}{|l|}{ Canthon Hoffmannsegg, 1817} \\
\hline 58. Canthon fuscipes Erichson, 1847 & $\begin{array}{l}\text { PIU, SULL, PAI, TAL, SECH, MORR) (BESII, BESc, } \\
\text { DES, MAN, BSI) }\end{array}$ & Juárez-Noé \& González-Coronado (2018) \\
\hline 59. Canthon sp. 1. & (AYA) (BHM) & Juárez-Noé \& González-Coronado (2018) \\
\hline \multicolumn{3}{|l|}{ Onoreidium Vaz de Mello, 2008} \\
\hline 60. Onoreidium cristatum (Arrow, 1931) & $(\mathrm{AYA}, \mathrm{HUA})(\mathrm{BHM})$ & Juárez-Noé \& González-Coronado (2018) \\
\hline \multicolumn{3}{|l|}{ Ontherus Erichson, 1847} \\
\hline 61. Ontherus (Caelontherus) howdeni Génier, 1996 & (AYA, HUA) (BHM) & Juárez-Noé \& González-Coronado (2018) \\
\hline 62. Ontherus (Planontherus) rectus Génier, 1996* & $(\mathrm{AYA}, \mathrm{HUA})(\mathrm{BHM})$ & Juárez-Noé \& González-Coronado (2018) \\
\hline \multicolumn{3}{|l|}{ Phanaeus MacLeay, 1819} \\
\hline $\begin{array}{l}\text { 63. Phanaeus (Phanaeus) lunaris Taschenberg, } \\
1870\end{array}$ & $(\mathrm{AYA}, \mathrm{HUA})(\mathrm{BHM})$ & Juárez-Noé \& González-Coronado (2018) \\
\hline \multicolumn{3}{|l|}{ Scatimus Erichson, 1847} \\
\hline 64. Scatimus monstrosus Balthasar, 1939 & (AYA) (BHM) & Juárez-Noé \& González-Coronado (2018) \\
\hline \multicolumn{3}{|l|}{ Aphodiinae Leach, 1815} \\
\hline \multicolumn{3}{|l|}{ Aphodius Illiger, 1798} \\
\hline $\begin{array}{l}\text { 65. Aphodius (Labarrus) pseudolividus Balthasar, } \\
1941\end{array}$ & $\begin{array}{l}\text { (PIU, SULL, PAI, TAL, SECH, MORR, AYA, HUA) } \\
\text { (BESII, BESc, BESm, DES, BSI, MAN, BHM) }\end{array}$ & \\
\hline \multicolumn{3}{|l|}{ Ataenius Harold, 1867} \\
\hline 66. Ataenius sp. 1. & (PIU, SULL, PAI, TAL, SECH) (BESII, DES) & Juárez-Noé \& González-Coronado (2018) \\
\hline \multicolumn{3}{|l|}{ Melolonthinae Leach, 1819} \\
\hline \multicolumn{3}{|l|}{ Plectris Le Peletier \& Audinet-Serville, 1828} \\
\hline 67. Plectris sp. 1. & (AYA) (BHM) & Juárez-Noé \& González-Coronado (2018) \\
\hline \multicolumn{3}{|l|}{ Phyllophaga Harris, 1827} \\
\hline 68. Phyllophaga sp. 1. & (AYA) (BHM) & Juárez-Noé \& González-Coronado (2018) \\
\hline \multicolumn{3}{|l|}{ Rutelinae MacLeay, 1819} \\
\hline \multicolumn{3}{|l|}{ Leucothyreus MacLeay, 1819} \\
\hline 69. Leucothyreus sp. 1. & (AYA) (BHM) & Juárez-Noé \& González-Coronado (2018) \\
\hline 70. Leucothyreus sp. 2. & (PIU, SULL, SECH, TAL, PAI) (BES, DES) & Juárez-Noé \& González-Coronado (2018) \\
\hline \multicolumn{3}{|l|}{ Platycoelia Dejean, 1833} \\
\hline 71. Platycoelia marginata Burmeister, 1844 & (HUA, AYA) (BSI, BHM) & \\
\hline \multicolumn{3}{|l|}{ Paranomala Casey, 1915} \\
\hline $\begin{array}{l}\text { 72. Paranomala undulata peruviana Guérin- } \\
\text { Méneville, } 1831\end{array}$ & $\begin{array}{l}\text { (PIU, SULL, TAL, PAI, SECH, MORR, AYA, HUA) } \\
\text { (BESII, BESc, BESm, DES, BSI, BHM) }\end{array}$ & Juárez-Noé \& González-Coronado (2018) \\
\hline \multicolumn{3}{|l|}{ Strigoderma Burmeister, 1844} \\
\hline 73. Strigoderma sp. 1. & (PIU) (BESII) & Juárez-Noé \& González-Coronado (2018) \\
\hline Mecopelidnota F. Bates, 1904. & & \\
\hline
\end{tabular}


Tabla 1 (continuación).

\begin{tabular}{|c|c|c|}
\hline Taxones & Distribución (provincia) (paisaje ecológico) & Referencia \\
\hline 74. Mecopelidnota marxi Soula, 2008 & (PIU, SULL, HUA) (BESII, BSI, BHM) & Juárez-Noé \& González-Coronado (2018) \\
\hline 75. Mecopelidnota mezai Soula, $2008^{*}$ & (HUA) (BSI, BHM) & Juárez-Noé \& González-Coronado (2018) \\
\hline 76. Mecopelidnota arrowi Bates, 1904 & $(\mathrm{HUA})(\mathrm{BHM})$ & \\
\hline \multicolumn{3}{|l|}{ Homonyx Guérin-Méneville, 1839} \\
\hline 77. Homonyx maurettei Soula, $2010^{*}$ & $(\mathrm{HUA})(\mathrm{BHM})$ & Juárez-Noé \& González-Coronado (2018) \\
\hline \multicolumn{3}{|l|}{ Género Microrutela F. Bates, 1904} \\
\hline 78. Microrutela campa (Ohaus, 1922) $\dagger$ & (AYA) (BESc) & Jameson (1997) \\
\hline \multicolumn{3}{|l|}{ Thyriochlorata Ohaus, 1915} \\
\hline 79. Thyriochlorata villosa (Ohaus, 1908$)^{*} \dagger$ & (HUA) (BHM) & Soula (2002) \\
\hline \multicolumn{3}{|l|}{ Chrysophora Dejean, 1821} \\
\hline 80. Chrysophora chrysochlora (Latreille, 1811)† & $(\mathrm{AYA})(\mathrm{BHM})$ & \\
\hline \multicolumn{3}{|l|}{ Dynastinae MacLeay, 1819} \\
\hline \multicolumn{3}{|l|}{ Cyclocephala Dejean, 1821} \\
\hline 81. Cyclocephala sp. 1. & $\begin{array}{l}\text { (PIU, SULL, PAI, TAL, MORR, SECH, AYA, HUA) } \\
\text { (BESII, BESc, BESm, DES, BSI, BHM) }\end{array}$ & Juárez-Noé \& González-Coronado (2018) \\
\hline 82. Cyclocephala macrophylla Erichson, $1847 \dagger$ & $(\mathrm{BHM})$ & Moore et al., (2018) \\
\hline \multicolumn{3}{|l|}{ Ancognatha Erichson, 1847} \\
\hline 83. Ancognatha scarabaeoides Erichson, 1847 & (AYA, HUA) (BSI, BHM) & Juárez-Noé \& González-Coronado (2018) \\
\hline 84. Ancognatha vulgaris Arrow, 1911 & (AYA, HUA) (BSI, BHM) & Juárez-Noé \& González-Coronado (2018) \\
\hline \multicolumn{3}{|l|}{ Tomarus Erichson, 1847} \\
\hline 85. Tomarus maimon Erichson, 1847 & $\begin{array}{l}\text { (PIU, SULL, TAL, PAI, SECH, MORR, HUA, AYA) } \\
\text { (BESII, BESc, BESm, DES, MAN, BSI) }\end{array}$ & Juárez-Noé \& González-Coronado (2018) \\
\hline 86. Tomarus peruvianus (Endrödi, 1970) & $\begin{array}{l}\text { (PIU, SULL, TAL, PAI, SECH, MORR, HUA, AYA) } \\
\text { (BESII, BESc, BESm, DES) }\end{array}$ & \\
\hline \multicolumn{3}{|l|}{ Golofa Hope, 1837} \\
\hline 87. Golofa eacus Burmeister, 1847 & $(H U A, A Y A)(B H M)$ & Juárez-Noé \& González-Coronado (2018) \\
\hline 88. Golofa limogesi Ratcliffe \& Le Tirant, 2018 & (HUA, AYA) (BHM) & Juárez-Noé \& González-Coronado (2018) \\
\hline 89. Golofa aegeon (Drury, 1773) & $(\mathrm{HUA})(\mathrm{BHM})$ & \\
\hline \multicolumn{3}{|l|}{ Megaceras Hope, 1837} \\
\hline 90. Megaceras morpheus Burmeister, 1847 & $(\mathrm{HUA}, \mathrm{AYA})(\mathrm{BHM})$ & Juárez-Noé \& González-Coronado (2018) \\
\hline \multicolumn{3}{|l|}{ Strategus Kirby, 1828} \\
\hline 91. Strategus aloeus (Linnaeus, 1758) & (MORR, AYA, HUA) (BHM) & Juárez-Noé \& González-Coronado (2018) \\
\hline \multicolumn{3}{|l|}{ Heterogomphus Burmeister, 1847} \\
\hline 92. Heterogomphus rubripennis Prell, 1912 & $(\mathrm{AYA})(\mathrm{BHM})$ & Juárez-Noé \& González-Coronado (2018) \\
\hline \multicolumn{3}{|l|}{ Coelosis Hope, 1837} \\
\hline 93. Coelosis biloba (Linnaeus, 1767) & (HUA, AYA) (BHM) & Juárez-Noé \& González-Coronado (2018) \\
\hline \multicolumn{3}{|l|}{ Phileurus Latreille, 1807} \\
\hline 94. Phileurus valgus (Olivier, 1789) & (PIU) (BESII) & Juárez-Noé \& González-Coronado (2018) \\
\hline \multicolumn{3}{|l|}{ Lycomedes Breme, 1844} \\
\hline 95. Lycomedes ohausi Arrow, 1908 & $(\mathrm{HUA})(\mathrm{BHM})$ & Juárez-Noé \& González-Coronado (2018) \\
\hline \multicolumn{3}{|l|}{ Dynastes Kirby, 1825} \\
\hline 96. Dynastes neptunus (Quensel, 1806) & (HUA) (BHM) & \\
\hline \multicolumn{3}{|l|}{ Megasoma Kirby, 1825} \\
\hline 97.Megasoma actaeon (Linnaeus, 1758) & (MORR) (BHM) & \\
\hline \multicolumn{3}{|l|}{ Cetoniinae Leach, 1815} \\
\hline \multicolumn{3}{|l|}{ Gymnetis MacLeay, 1819} \\
\hline 98. Gymnetis stellata Latreille, 1833 & (PIU) (BESII) & Juárez-Noé \& González-Coronado (2018) \\
\hline 99. Gymnetis merops Ratcliffe, $2018^{*}$ & (PIU, SULL, PAI, TAL, SECH, MORR) (BESII, BESc) & \\
\hline \multicolumn{3}{|l|}{ Marmarina Kirby, 1827} \\
\hline 100. Marmarina maculosa (Olivier, 1789)† & (AYA) (BESc) & Ratcliffe (2015) \\
\hline \multicolumn{3}{|l|}{ Hoplopyga Thomson, 1880} \\
\hline 101. Hoplopyga liturata (Olivier, 1789) & (HUA) (BSI) & Juárez-Noé \& González-Coronado (2018) \\
\hline Buprestidae Leach, 1815 & & \\
\hline
\end{tabular}


Tabla 1 (continuación).

\begin{tabular}{|c|c|c|}
\hline Taxones & Distribución (provincia) (paisaje ecológico) & Referencia \\
\hline \multicolumn{3}{|l|}{ Polycestinae Lacordaire, 1857} \\
\hline \multicolumn{3}{|l|}{ Polycesta Dejean, 1833} \\
\hline $\begin{array}{l}\text { 102. Polycesta (Nemaphorus) excavata } \\
\text { Blanchard, } 1846\end{array}$ & $\begin{array}{l}\text { (PIU, SULL, TAL, PAI, MORR, SECH) (BESII, BESc, } \\
\text { BESm, DES) }\end{array}$ & Juárez-Noé \& González-Coronado (2018) \\
\hline \multicolumn{3}{|l|}{ Hayekina Cobos, 1980} \\
\hline 103. Hayekina dispar (Kerremans, 1899) & (PIU, SULL, TAL) (BESII) & Juárez-Noé \& González-Coronado (2018) \\
\hline \multicolumn{3}{|l|}{ Buprestinae Leach, 1815} \\
\hline \multicolumn{3}{|l|}{ Agrilaxia Kerremans, 1903} \\
\hline 104. Agrilaxia acuminata Cobos, $1972^{*}$ & $\begin{array}{l}\text { (PIU, SULL, PAI, TAL, MORR, SECH) (BESII, BESc, } \\
\text { BESm, DES) }\end{array}$ & Juárez-Noé \& González-Coronado (2018) \\
\hline \multicolumn{3}{|l|}{ Chrysobothris Eschscholtz, 1829} \\
\hline 105. Chrysobothris sp. 1. & $\begin{array}{l}\text { (PIU, SULL, PAI, TAL, MORR, SECH) (BESII, BESc, } \\
\text { BESm, DES) }\end{array}$ & Juárez-Noé \& González-Coronado (2018) \\
\hline \multicolumn{3}{|l|}{ Lasionota Mannerheim, 1837} \\
\hline $\begin{array}{l}\text { 106. Lasionota (Nelsonozodes) piurae Moore \& } \\
\text { Diéguez, } 2019^{*} \dagger\end{array}$ & (PIU) (BESII) & Moore \& Diéguez (2019) \\
\hline \multicolumn{3}{|l|}{ Agrilinae Laporte, 1835} \\
\hline \multicolumn{3}{|l|}{ Agrilus Curtis, 1825} \\
\hline 107. Agrilus piuraensis Juárez \& González, 2017 & (PIU) (BESII) & Juárez-Noé \& González-Coronado (2018) \\
\hline 108. Agrilus sp. 1. & (PIU) (BESII) & Juárez-Noé \& González-Coronado (2018) \\
\hline 109. Agrilus sp. 1. & (PIU) (BESII) & Juárez-Noé \& González-Coronado (2018) \\
\hline \multicolumn{3}{|l|}{ Paragrilus Saunders, 1871} \\
\hline 110. Paragrilus sp. 1 & (PIU) (BESII) & Juárez-Noé \& González-Coronado (2018) \\
\hline \multicolumn{3}{|l|}{ Chrysochroinae Laporte, 1835} \\
\hline \multicolumn{3}{|l|}{ Pseudolampetis Obenberger, 1926} \\
\hline 111. Pseudolampetis bilineata (Latreille, 1813) & (SULL) (BESII) & Juárez-Noé \& González-Coronado (2018) \\
\hline \multicolumn{3}{|l|}{ Elateridae Leach, 1815} \\
\hline \multicolumn{3}{|l|}{ Agrypninae Candèze, 1857} \\
\hline \multicolumn{3}{|l|}{ Chalcolepidius Eschscholtz, 1829} \\
\hline 112. Chalcolepidius fryi Candèze, $1874^{*}$ & (PIU, SECH) (BESII, DES) & Juárez-Noé \& González-Coronado (2018) \\
\hline 113. Chalcolepidius corpulentus Candèze, 1874 & $(\mathrm{TAL})(\mathrm{BESII})$ & Casari (2002) \\
\hline \multicolumn{3}{|l|}{ Aeolus Leach, 1815} \\
\hline 114. Aeolus sp. 1. & (PIU, SULL, SECH) (BESII, DES, MAN) & Juárez-Noé \& González-Coronado (2018) \\
\hline \multicolumn{3}{|l|}{ Heteroderes Latreille, 1834} \\
\hline 115. Heteroderes amplicollis (Gyllenhal, 1817) & (PIU, SECH) (BESII, DES, MAN) & Juárez-Noé \& González-Coronado (2018) \\
\hline \multicolumn{3}{|l|}{ Conoderus Eschscholtz, 1829} \\
\hline 116. Conoderus sp. 1. & $\begin{array}{l}\text { (PIU, SULL, PAI, SECH, TAL, MORR) (BESII, BESc, } \\
\text { DES) }\end{array}$ & Juárez-Noé \& González-Coronado (2018) \\
\hline 117. Conoderus sp. 1. & (PIU) (BESII) & Juárez-Noé \& González-Coronado (2018) \\
\hline 118. Conoderus repandus (Erichson, 1847)† & (PIU) (BESII) & \\
\hline \multicolumn{3}{|l|}{ Semiotinae Jakobson, 1913} \\
\hline \multicolumn{3}{|l|}{ Semiotus Eschscholtz, 1829} \\
\hline 119. Semiotus angustus Wells, 2017 & (AYA) (BHM) & Juárez-Noé \& González-Coronado (2018) \\
\hline \multicolumn{3}{|l|}{ Cardiophorinae Candéze, 1859} \\
\hline \multicolumn{3}{|l|}{ Horistonotus Candéze, 1860} \\
\hline 120. Horistonotus sp. 1. & (PIU, SECH) (BESII, DES) & Juárez-Noé \& González-Coronado (2018) \\
\hline \multicolumn{3}{|l|}{ Lycidae Laporte, 1836} \\
\hline Lycinae Laporte, 1836 & & \\
\hline Calopteron Laporte, 1838 & & \\
\hline 121. Calopteron sp. 1. & $(\mathrm{AYA}, \mathrm{HUA})(\mathrm{BHM}, \mathrm{BSI})$ & Juárez-Noé \& González-Coronado (2018) \\
\hline Plateros Bourgeois, 1879 & & \\
\hline 122. Plateros sp. 1 & (PIU) (BESII) & Juárez-Noé \& González-Coronado (2018) \\
\hline Lampyridae Rafinesque, 1815 & & \\
\hline
\end{tabular}


Tabla 1 (continuación).

\begin{tabular}{|c|c|c|}
\hline Taxones & Distribución (provincia) (paisaje ecológico) & Referencia \\
\hline \multicolumn{3}{|l|}{ Amydetinae Olivier, 1907} \\
\hline \multicolumn{3}{|l|}{ Cladodes Solier, 1849} \\
\hline 123. Cladodes sp. 1 & (AYA) (BHM) & Juárez-Noé \& González-Coronado (2018) \\
\hline \multicolumn{3}{|l|}{ Lampyrinae Rafinesque, 1815} \\
\hline \multicolumn{3}{|l|}{ Photinus Laporte, 1833} \\
\hline 124. Photinus baeri (Oliver, 1907) & (PIU) (BESII) & Juárez-Noé \& González-Coronado (2018) \\
\hline \multicolumn{3}{|l|}{ Cantharidae Imhoff, 1856 (1815) } \\
\hline \multicolumn{3}{|l|}{ Silinae Mulsant, 1862} \\
\hline \multicolumn{3}{|l|}{ Discodon Gorham, 1881} \\
\hline 125. Discodon sp. 1. & (AYA) (BHM) & Juárez-Noé \& González-Coronado (2018) \\
\hline \multicolumn{3}{|l|}{ Silis Charpentier, 1825} \\
\hline 126. Silis foveolata Kirsch, $1865+$ & (SULL) (BESII) & Constantin (2011) \\
\hline \multicolumn{3}{|l|}{ Chauliognathinae LeConte, 1861} \\
\hline \multicolumn{3}{|l|}{ Chauliognathus Hentz, 1830} \\
\hline 127. Chauliognathus heros Guérin-Méneville, 1843 & $(\mathrm{HUA})(\mathrm{BSI})$ & Juárez-Noé \& González-Coronado (2018) \\
\hline \multicolumn{3}{|l|}{ Dermestidae Latreille, 1804} \\
\hline \multicolumn{3}{|l|}{ Dermestinae Latreille, 1804} \\
\hline \multicolumn{3}{|l|}{ Dermestes Linnaeus, 1758} \\
\hline $\begin{array}{l}\text { 128. Dermestes (Dermestes) peruvianus Laporte, } \\
1840\end{array}$ & (AYA, HUA) (BSI, BHM) & Juárez-Noé \& González-Coronado (2018) \\
\hline $\begin{array}{l}\text { 129. Dermestes (Dermestinus) maculatus } \\
\text { DeGeer, } 1774\end{array}$ & (PIU) (BESII) & \\
\hline $\begin{array}{l}\text { 130. Dermestes (Dermestinus) frischii Kugellan, } \\
\text { 1792† }\end{array}$ & (PIU) (BESII) & \\
\hline 131. Dermestes (Dermestes) ater DeGeer, 1774† & (PIU) (BESII) & \\
\hline \multicolumn{3}{|l|}{ Attageninae Laporte, 1840} \\
\hline \multicolumn{3}{|l|}{ Attagenus Latreille, 1802} \\
\hline $\begin{array}{l}\text { 132. Attagenus (Attagenus) fasciatus (Thunberg, } \\
\text { 1795) }\end{array}$ & $\begin{array}{l}\text { (PIU, SULL, TAL, SECH, PAI, MORR, HUA, AYA) } \\
\text { (BESII, BESc, BESm, DES, BSI, BHM) }\end{array}$ & Juárez-Noé \& González-Coronado (2018) \\
\hline \multicolumn{3}{|l|}{ Megatominae Leach, 1815} \\
\hline \multicolumn{3}{|l|}{ Trogoderma Dejean, 1821} \\
\hline 133. Trogoderma sp. 1. & (PIU) (BESII) & \\
\hline Bostrichidae Latreille, 1802 & & Juárez-Noé \& González-Coronado (2018) \\
\hline \multicolumn{3}{|l|}{ Bostrichinae Latreille, 1802} \\
\hline \multicolumn{3}{|l|}{ Dolichobostrychus Lesne, 1899} \\
\hline 134. Dolichobostrychus angustus Steinheil, 1872 & (PIU) (BESII) & Juárez-Noé \& González-Coronado (2018) \\
\hline \multicolumn{3}{|l|}{ Amphicerus LeConte, 1861} \\
\hline $\begin{array}{l}\text { 135. Amphicerus (Amphicerus) cornutus (Pallas, } \\
\text { 1772) }\end{array}$ & (PIU, SULL) (BESII) & Juárez-Noé \& González-Coronado (2018) \\
\hline \multicolumn{3}{|l|}{ Micrapate Casey, 1898} \\
\hline 136. Micrapate scabrata (Erichson, 1847) & (PIU, SULL, TAL) (BESII) & Juárez-Noé \& González-Coronado (2018) \\
\hline \multicolumn{3}{|l|}{ Sinoxylon Duftschmid, 1825} \\
\hline 137. Sinoxylon unidentatum Fabricius, 1801 & (PIU) (BESII) & Juárez-Noé \& González-Coronado (2018) \\
\hline 138. Sinoxylon anale Lesne, 1897 & (PIU, MORR) (BESII, BESc) & \\
\hline \multicolumn{3}{|l|}{ Dinoderinae Thomson, 1863} \\
\hline Dinoderus Stephens, 1830 & & \\
\hline 139. Dinoderus minutus (Fabricius, 1775) & (PIU) (BESII) & Juárez-Noé \& González-Coronado (2018) \\
\hline Ptinidae Latreille, 1802 & & \\
\hline Ptininae Latreille, 1802 & & \\
\hline Ptinus Linnaeus, 1767 & & \\
\hline 140. Ptinus sp. 1. & (PIU, TAL) (BESII) & Juárez-Noé \& González-Coronado (2018) \\
\hline Gibbium Scopoli, 1777 & & \\
\hline 141. Gibbium psylloides (Czenpinski, 1778) & (TAL) (BESII) & Juárez-Noé \& González-Coronado (2018) \\
\hline
\end{tabular}


Tabla 1 (continuación).

\section{Taxones}

Xyletininae Gistel, 1848

Lasioderma Stephens, 1835

142. Lasioderma serricorne Fabricius, 1792

Anobiinae Kirby, 1837

Stegobium Motschulsky, 1860

143. Stegobium paniceum (Linnaeus, 1758)†

Trogossitidae Latreille, 1802

Trogossitinae Latreille, 1802

Airora Reitter, 1876

144. Airora sp. 1

Tenebroides Piller \& Mitterpacher, 1783

145. Tenebroides sp. 1

Cleridae Latreille, 1802

Korynetinae Laporte, 1836

Necrobia Olivier, 1795

146. Necrobia rufipes (DeGeer, 1775)

Neorthopleurinae Opitz, 2009

Neorthopleura Barr, 1976

147. Neorthopleura guayasensis Opitz, 2013

Tillinae Leach, 1835

Cymatodera Gray, 1832

148. Cymatodera sp. 1.

Hydnocerinae Spinola, 1844

Phyllobaenus Dejean, 1833

149. Phyllobaenus sp. 1.

Peloniinae Latreille, 1802

Pelonium Spinola, 1844

150. Pelonium riveti Lesne, 1909†

151. Pelonium anteguttatum Opitz, $2018 \dagger$

Melyridae Leach, 1815

Melyrinae Leach, 1815

Astylus Laporte de Castelnau, 1836

152. Astylus longulus Constantin, 2011

153. Astylus lojaensis Constantin, 2011

154. Astylus bonplandi Erichson, 1847†

Silvanidae Kirby, 1837

Silvaninae Kirby, 1837

Oryzaephilus Ganglbauer, 1899

155. Oryzaephilus mercator (Fauvel, 1889) (PIU) (BESII)

156. Oryzaephilus surinamensis (Linnaeus, 1758) (PIU) (BESII)

Género Ahasverus Gozis, 1881

157. Ahasverus advena (Waltl, 1834)†

Passandridae Blanchard, 1845

Taphroscelidia Crotch, 1873

158. Taphroscelidia postica (Grouvelle, 1916)

Nitidulidae Latreille, 1802

Nitidulinae Latreille, 1802

Lobiopa Erichson, 1834

159. Lobiopa sp. 1

(PIU, SULL, TAL, HUA, AYA) (BESII, BSI, BHM)

(PIU) (BESII)

(SULL) (BESII)

Juárez-Noé \& González-Coronado (2018)

Juárez-Noé \& González-Coronado (2018) Juárez-Noé \& González-Coronado (2018)

Cychramus Kugelann, 179

160. Cychramus sp. 1.
(AYA) (BHM)

(HUA) (BHM)

, SECH, SULL, MORR, HUA, AYA) (BESII, BESc, BESm, DES, BSI, BHM)

(PAI) (BESII)

(SULL) (BESII)

Juárez-Noé \& González-Coronado (2018) Juárez-Noé \& González-Coronado (2018) Constantin (2011)

Graellsia, 75(2), diciembre 2019, e097 - ISSN-L: 0367-5041 - https://doi.org/10.3989/graellsia.2019.v75.233 
Tabla 1 (continuación).

\begin{tabular}{|c|c|c|}
\hline Taxones & Distribución (provincia) (paisaje ecológico) & Referencia \\
\hline \multicolumn{3}{|l|}{ Carpophilinae Erichson, 1842} \\
\hline \multicolumn{3}{|l|}{ Carpophilus Stephens, 1830} \\
\hline 161. Carpophilus sp. 1. & (PIU, SULL, HUA, AYA) (BESII, BSI, BHM) & Juárez-Noé \& González-Coronado (2018) \\
\hline \multicolumn{3}{|l|}{ Cillaeinae Kirejtshuk \& Audisio, 1986} \\
\hline \multicolumn{3}{|l|}{ Colopterus Erichson, 1842} \\
\hline 162. Colopterus sp. 1. & (PIU, SULL) (BESII) & Juárez-Noé \& González-Coronado (2018) \\
\hline \multicolumn{3}{|l|}{ Coccinellidae Latreille, 1807} \\
\hline \multicolumn{3}{|l|}{ Coccinellinae Latreille, 1807} \\
\hline \multicolumn{3}{|l|}{ Azya Mulsant, 1850} \\
\hline 163. Azya orbigera ecuadorica Gordon, 1980 & (PIU, SULL, TAL, PAI) (BESII) & Juárez-Noé \& González-Coronado (2018) \\
\hline \multicolumn{3}{|l|}{ Delphastus Casey, 1899} \\
\hline 164. Delphastus berryi Gordon, $1994 \dagger$ & (SULL) (TAL) (BESII) & Juárez-Noé \& González-Coronado (2018) \\
\hline \multicolumn{3}{|l|}{ Scymnus Kugelann, 1794} \\
\hline $\begin{array}{l}\text { 165. Scymnus (Pullus) rubicundus Erichson, } \\
1847\end{array}$ & $\begin{array}{l}\text { (PIU, SULL, TAL, PAI, MORR, SECH, HUA) (BESII, } \\
\text { BESc, DES, MAN, BSI) }\end{array}$ & Juárez-Noé \& González-Coronado (2018) \\
\hline 166. Scymnus (Pullus) loewii Mulsant, 1850 & (PIU, SECH) (BESII, DES) & Juárez-Noé \& González-Coronado (2018) \\
\hline \multicolumn{3}{|l|}{ Scymnobius Casey, 1899} \\
\hline $\begin{array}{l}\text { 167. Scymnobius galapagoensis (Waterhouse, } \\
\text { 1845) }\end{array}$ & $\begin{array}{l}\text { (PIU, SULL, TAL, PAI, SECH, MORR, HUA) (BESII, } \\
\text { BESC, DES, MAN, BSI) }\end{array}$ & Juárez-Noé \& González-Coronado (2018) \\
\hline $\begin{array}{l}\text { 168. Scymnobius triangularis Gordon \& } \\
\text { González, } 2002\end{array}$ & (PIU) (BESII) & Juárez-Noé \& González-Coronado (2018) \\
\hline $\begin{array}{l}\text { 169. Scymnobius ecuadoricus Gordon \& } \\
\text { González, 2005† }\end{array}$ & (MORR) (BESc) & \\
\hline \multicolumn{3}{|l|}{ Stethorus Weise, 1885} \\
\hline $\begin{array}{l}\text { 170. Stethorus grossepunctatus (Gordon \& } \\
\text { Chapin, 1983) }\end{array}$ & (MORR) (BESII) & Juárez-Noé \& González-Coronado (2018) \\
\hline $\begin{array}{l}\text { 171. Stethorus peruvianus González, Gordon \& } \\
\text { Robinson, } 2008^{*}\end{array}$ & (PIU, MORR) (BESII) & Juárez-Noé \& González-Coronado (2018) \\
\hline \multicolumn{3}{|l|}{ Parastethorus Pang \& Mao, 1975} \\
\hline 172. Parastethorus histrio (Chazeau, 1974) & (MORR) (BESII) & Juárez-Noé \& González-Coronado (2018) \\
\hline \multicolumn{3}{|l|}{ Nephaspis Casey, 1899} \\
\hline 173. Nephaspis sp. 1. & (PIU) (BESII) & Juárez-Noé \& González-Coronado (2018) \\
\hline \multicolumn{3}{|l|}{ Pentilia Mulsant, 1850} \\
\hline 174. Pentilia sp & (PIU, SULL, PAI, MORR) (BESII, BESc) & Juárez-Noé \& González-Coronado (2018) \\
\hline \multicolumn{3}{|l|}{ Hyperaspis Chevrolat, 1836} \\
\hline 175. Hyperaspis onerata Mulsant, 1850 & (PIU, SULL, PAI, TAL, SECH, MORR) (BESII, BESc) & Juárez-Noé \& González-Coronado (2018) \\
\hline $\begin{array}{l}\text { 176. Hyperaspis esmeraldas Gordon \& González, } \\
2011\end{array}$ & (PIU, SULL) (BESII) & Juárez-Noé \& González-Coronado (2018) \\
\hline 177. Hyperaspis arida Gordon \& Canepari, 2008* & $(\mathrm{PIU})(\mathrm{BESII})$ & Juárez-Noé \& González-Coronado (2018) \\
\hline \multicolumn{3}{|l|}{ Brachiacantha Dejean, 1837} \\
\hline $\begin{array}{l}\text { 178. Brachiacantha darlene Gordon \& Canepari, } \\
2014\end{array}$ & (PIU, TAL, SECH, MORR, SULL) (BESII, BESc) & \\
\hline \multicolumn{3}{|l|}{ Zagreus Mulsant, 1850} \\
\hline 179. Zagreus hexasticta (Crotch, 1874)* & $\begin{array}{l}\text { (PIU, SULL, PAI, TAL, SECH, MORR) (BESII, BESc, } \\
\text { DES) }\end{array}$ & Juárez-Noé \& González-Coronado (2018) \\
\hline 180. Zagreus decempunctatus (Weise, 1893) & (MORR) (BESII) & Juárez-Noé \& González-Coronado (2018) \\
\hline \multicolumn{3}{|l|}{ Psyllobora Chevrolat, 1837} \\
\hline 181. Psyllobora confluens Fabricius, 1801 & $\begin{array}{l}\text { (PIU, SULL, TAL, PAI, SECH, MORR, HUA) (BESII, } \\
\text { BESc, BSI, DES, BHM) }\end{array}$ & Juárez-Noé \& González-Coronado (2018) \\
\hline 182. Psyllobora sp. 1. & (PIU, SULL) (BESII) & Juárez-Noé \& González-Coronado (2018) \\
\hline 183. Psyllobora sp. 2. & (PIU) (BESII) & Juárez-Noé \& González-Coronado (2018) \\
\hline \multicolumn{3}{|l|}{ Coleomegilla Timberlake, 1920} \\
\hline $\begin{array}{l}\text { 184. Coleomegilla maculata bisexpunctata } \\
\text { Latreille, } 1833\end{array}$ & (PIU, SULL, PAI) (BESII) & Juárez-Noé \& González-Coronado (2018) \\
\hline
\end{tabular}


Tabla 1 (continuación).

\begin{tabular}{|c|c|c|}
\hline Taxones & Distribución (provincia) (paisaje ecológico) & Referencia \\
\hline \multicolumn{3}{|l|}{ Eriopis Mulsant, 1850} \\
\hline 185. Eriopis sp. 1. & (AYA) (BHM) & Juárez-Noé \& González-Coronado (2018) \\
\hline \multicolumn{3}{|l|}{ Cycloneda Crotch, 1871} \\
\hline 186. Cycloneda sanguinea (Linnaeus, 1763) & $\begin{array}{l}\text { (PIU, SULL, TAL, PAI, SECH, MORR, AYA, HUA) } \\
\text { (BESII, BESc, BESm, DES, MAN, BSI, BHM) }\end{array}$ & Juárez-Noé \& González-Coronado (2018) \\
\hline \multicolumn{3}{|l|}{ Cheilomenes Dejean, 1836} \\
\hline 187. Cheilomenes sexmaculata Fabricius, 1781 & $\begin{array}{l}\text { (PIU, SULL, SECH, TAL, PAI, MORR) (BESII, BESc, } \\
\text { DES, MAN) }\end{array}$ & Juárez-Noé \& González-Coronado (2018) \\
\hline \multicolumn{3}{|l|}{ Paraneda Timberlake, 1943} \\
\hline 188. Paraneda pallidula guticollis Mulsant, 1850 & $\begin{array}{l}\text { (PIU, SULL, TAL, PAI, SECH, MORR, AYA, HUA) } \\
\text { (BESII, BESc, BESm, DES, MAN, BSI, BHM) }\end{array}$ & Juárez-Noé \& González-Coronado (2018) \\
\hline 189. Paraneda sp. 1. & $\begin{array}{l}\text { (PIU, SULL, TAL, PAI, SECH, MORR) (BESII, BESc, } \\
\text { DES, MAN) }\end{array}$ & Juárez-Noé \& González-Coronado (2018) \\
\hline \multicolumn{3}{|l|}{ Harmonia Mulsant, 1846} \\
\hline 190. Harmonia axyridis (Pallas, 1772) & $\begin{array}{l}\text { (PIU, SECH, SULL, PAI, MORR, TAL) (BESII, BESc, } \\
\text { DES) }\end{array}$ & \\
\hline \multicolumn{3}{|l|}{ Hippodamia Dejean, 1837} \\
\hline $\begin{array}{l}\text { 191. Hippodamia convergens Guerin-Meneville, } \\
1836\end{array}$ & $\begin{array}{l}\text { (PIU, SULL, TAL, PAI, SECH, MORR, AYA, HUA) } \\
\text { (BESII, BESc, BESm, DES, BSI, MAN, BHM) }\end{array}$ & Juárez-Noé \& González-Coronado (2018) \\
\hline \multicolumn{3}{|l|}{ Neda Mulsant, 1850} \\
\hline 192. Neda aequatoriana Mulsant, 1853 & (AYA, HUA) (BHM, BSI) & Juárez-Noé \& González-Coronado (2018) \\
\hline \multicolumn{3}{|l|}{ Epilachna Chevrolat, 1837} \\
\hline 193. Epilachna flavofasciata (LaPorte, 1840) & (AYA) (BHM) & Juárez-Noé \& González-Coronado (2018) \\
\hline 194. Epilachna ambigua Mader, 1958 & (AYA) (BHM) & Juárez-Noé \& González-Coronado (2018) \\
\hline 195. Epilachna viridilineata Crotch, 1874 & (AYA) (BHM) & Juárez-Noé \& González-Coronado (2018) \\
\hline 196. Epilachna discolor Erichson, 1847 & (AYA) (BHM) & Juárez-Noé \& González-Coronado (2018) \\
\hline \multicolumn{3}{|l|}{ Rodolia Mulsant, 1850} \\
\hline 197. Rodolia cardinalis (Mulsant, 1850)† & (PIU) (BESII) & \\
\hline \multicolumn{3}{|l|}{ Ripiphoridae Gemminger, 1870} \\
\hline \multicolumn{3}{|l|}{ Ripiphorinae Gemminger, 1870} \\
\hline \multicolumn{3}{|l|}{ Macrosiagon Hentz, 1830} \\
\hline $\begin{array}{l}\text { 198. Macrosiagon octomaculata (Gerstaecker, } \\
\text { 1855) }\end{array}$ & (PIU, SULL) (BESII) & Juárez-Noé \& González-Coronado (2018) \\
\hline \multicolumn{3}{|l|}{ Mordellidae Latreille, 1802} \\
\hline \multicolumn{3}{|l|}{ Mordellinae Latreille, 1802} \\
\hline \multicolumn{3}{|l|}{ Mordella Linnaeus, 1758} \\
\hline 199. Mordella sp† & (PIU) (BESII) & \\
\hline \multicolumn{3}{|l|}{ Tenebrionidae Latreille, 1802} \\
\hline \multicolumn{3}{|l|}{ Pimeliinae Latreille, 1802} \\
\hline \multicolumn{3}{|l|}{ Psammetichus Latreille, 1804} \\
\hline $\begin{array}{l}\text { 200. Psammetichus costatus Guérin-Méneville, } \\
1830\end{array}$ & $\begin{array}{l}\text { (PIU, SULL, PAI, SECH, MORR, TAL) (BESII, BESc, } \\
\text { DES, MAN) }\end{array}$ & Juárez-Noé \& González-Coronado (2018) \\
\hline 201. Psammetichus dissimilis Peña, $1994^{*}$ & $\begin{array}{l}\text { (PIU, SULL, TAL, PAI, MORR, SECH) (BESII, BESc, } \\
\text { DES, MAN) }\end{array}$ & Juárez-Noé \& González-Coronado (2018) \\
\hline \multicolumn{3}{|l|}{ Philorea Erichson, 1834} \\
\hline 202. Philorea mucronata Lesne, 1911 & (SECH) (DES) & Juárez-Noé \& González-Coronado (2018) \\
\hline \multicolumn{3}{|l|}{ Parapraocis Kulzer, 1958} \\
\hline 203. Parapraocis vagecostata (Fairmaire, 1902) & (TAL) (BESc) & \\
\hline \multicolumn{3}{|l|}{ Parepitragus Casey, 1907} \\
\hline $\begin{array}{l}\text { 204. Parepitragus macrophthalmus Marcuzzi, } \\
\text { 1961* }\end{array}$ & $\begin{array}{l}\text { (PIU, SULL, TAL, PAI, SECH, MORR) (BESII, BESc, } \\
\text { DES, MAN) }\end{array}$ & Juárez-Noé \& González-Coronado (2018) \\
\hline 205. Parepitragus pulverulentus Erichson, $1847 \dagger$ & $\begin{array}{l}\text { (PIU, SULL, TAL, PAI, SECH, MORR, AYA, HUA) } \\
\text { (BESII, BESc, DES, MAN) }\end{array}$ & \\
\hline Epitragopsis Casey, 1907 & & \\
\hline
\end{tabular}


Tabla 1 (continuación).

\begin{tabular}{|c|c|c|}
\hline Taxones & Distribución (provincia) (paisaje ecológico) & Referencia \\
\hline 206. Epitragopsis sp. 1. & $\begin{array}{l}\text { (PIU, SULL, TAL, PAI, SECH, MORR) (BESII, BESc, } \\
\text { DES) }\end{array}$ & Juárez-Noé \& González-Coronado (2018) \\
\hline \multicolumn{3}{|l|}{ Sechuranus Flores \& Giraldo, 2019} \\
\hline 207. Sechuranus barbatus (Kaszab, 1964)* & $\begin{array}{l}\text { (PIU, SULL, PAI, TAL, SECH, MORR) (BESII, BESc, } \\
\text { DES, MAN) }\end{array}$ & Juárez-Noé \& González-Coronado (2018) \\
\hline \multicolumn{3}{|l|}{ Kocakia Kaszab, 1981} \\
\hline 208. Kocakia opaca (Kaszab, 1982)* & $\begin{array}{l}\text { (PIU, SECH, SULL, TAL, PAI, MORR) (BESII, BESc, } \\
\text { DES) }\end{array}$ & Juárez-Noé \& González-Coronado (2018) \\
\hline \multicolumn{3}{|l|}{ Aryenis Bates, 1868} \\
\hline 209 Aryenis sp. 1. & (TAL) (BESII) & Juárez-Noé \& González-Coronado (2018) \\
\hline \multicolumn{3}{|l|}{ Tenebrioninae Latreille, 1802} \\
\hline \multicolumn{3}{|l|}{ Blapstinus Waterhouse, 1845} \\
\hline 210. Blapstinus sp. 1. & (PIU, SULL, TAL, SECH) (BESII, DES) & Juárez-Noé \& González-Coronado (2018) \\
\hline 211. Blapstinus sp. 2 & (PIU, SULL, TAL, SECH) (BESII, DES) & Juárez-Noé \& González-Coronado (2018) \\
\hline \multicolumn{3}{|l|}{ Tribolium Macleay, 1825} \\
\hline 212. Tribolium castaneum (Herbst, 1797)† & (PIU) (BESII) & \\
\hline \multicolumn{3}{|l|}{ Alphitobius Stephens, 1832} \\
\hline 213. Alphitobius diaperinus (Panzer, 1797)† & $\begin{array}{l}\text { (PIU, SULL, TAL, PAI, SECH, MORR, HUA, AYA) } \\
\text { (BESII, BESc, BESm, BSI, BHM) }\end{array}$ & \\
\hline \multicolumn{3}{|l|}{ Alaetrinus Iwan, 1995} \\
\hline 214. Alaetrinus pullus (Sahlberg, 1823)† & $\begin{array}{l}\text { (PIU, SULL, TAL, PAI, SECH, MORR, HUA, AYA) } \\
\text { (BESII, BESc, BESm, DES) }\end{array}$ & \\
\hline \multicolumn{3}{|l|}{ Trichoton Hope, 1840} \\
\hline 215. Trichoton sp. 1 & (PIU) (BESII) & Juárez-Noé \& González-Coronado (2018) \\
\hline \multicolumn{3}{|l|}{ Ammophorus Guérin, 1830} \\
\hline 216. Ammophorus rubripes Solier, 1838 & $\begin{array}{l}\text { (PIU, SULL, PAI, SECH, MORR, TAL) (BESII, BESc, } \\
\text { DES, MAN) }\end{array}$ & Juárez-Noé \& González-Coronado (2018) \\
\hline 217. Ammophorus peruvianus Guerin, $1830 \dagger$ & $\begin{array}{l}\text { (PIU, SULL, TAL, PAI, SECH, MORR, AYA, HUA) } \\
\text { (BESII, BESc, DES, MAN) }\end{array}$ & \\
\hline \multicolumn{3}{|l|}{ Pumiliofossorum Silvestro \& Giraldo, 2015} \\
\hline $\begin{array}{l}\text { 218. Pumiliofossorum sechurae Giraldo \& Flores, } \\
\text { 2015* }\end{array}$ & (PIU, SECH) (BESII, DES) & Juárez-Noé \& González-Coronado (2018) \\
\hline \multicolumn{3}{|l|}{ Zophobas Dejean, 1834} \\
\hline $\begin{array}{l}\text { 219. Zophobas (Zophobas) opacus (Sahlberg, } \\
\text { 1823) }\end{array}$ & $\begin{array}{l}\text { (PIU, SULL, TAL, SECH, PAI, MORR) (BESII, BESc, } \\
\text { DES) }\end{array}$ & Juárez-Noé \& González-Coronado (2018) \\
\hline \multicolumn{3}{|l|}{ Ulomoides Blackburn, 1888} \\
\hline 220. Ulomoides dermestoides (Chevrolat, 1878) & (PIU) (BESII) & Juárez-Noé \& González-Coronado (2018) \\
\hline \multicolumn{3}{|l|}{ Diaperinae Latreille, 1802} \\
\hline \multicolumn{3}{|l|}{ Phaleria Latreille, 1804} \\
\hline 221. Phaleria pacifica Champion, 1886 & (PAI) (BESII) & Juárez-Noé \& González-Coronado (2018) \\
\hline $\begin{array}{l}\text { 222. Phaleria psammatea Giraldo-Mendoza, } \\
2019^{*} \dagger\end{array}$ & $(\mathrm{TAL})(\mathrm{DES})$ & Giraldo-Mendoza (2019) \\
\hline \multicolumn{3}{|l|}{ Oedemeridae Latreille, 1810} \\
\hline \multicolumn{3}{|l|}{ Oedemerinae Latreille, 1810} \\
\hline \multicolumn{3}{|l|}{ Oxycopis Arnett, 1951} \\
\hline 223. Oxycopis sp $†$ & (PIU) (BESII) & \\
\hline \multicolumn{3}{|l|}{ Meloidae Gyllenhal, 1810} \\
\hline \multicolumn{3}{|l|}{ Meloinae Gyllenhal, 1810} \\
\hline \multicolumn{3}{|l|}{ Lyttamorpha Kaszab, 1959} \\
\hline 224. Lyttamorpha reichenbachi Kirsch, 1866 & $(\mathrm{AYA})(\mathrm{BHM})$ & Juárez-Noé \& González-Coronado (2018) \\
\hline \multicolumn{3}{|l|}{ Spastomeloe Selander, 1985} \\
\hline 225. Spastomeloe singularis Selander, $1985^{*}$ & $(\mathrm{TAL})(\mathrm{DES})$ & Juárez-Noé \& González-Coronado (2018) \\
\hline Pseudopyrota Selander, 1990 & & \\
\hline
\end{tabular}


Tabla 1 (continuación).

\begin{tabular}{|c|c|c|}
\hline Taxones & Distribución (provincia) (paisaje ecológico) & Referencia \\
\hline $\begin{array}{l}\text { 226. Pseudopyrota sanguinithorax (Haag- } \\
\text { Rutenberg, 1880) }\end{array}$ & (PIU) (BESII) & Juárez-Noé \& González-Coronado (2018) \\
\hline \multicolumn{3}{|l|}{ Nemognathinae Laporte, 1840} \\
\hline \multicolumn{3}{|l|}{ Cissites Latreille, 1804} \\
\hline 227. Cissites maculata (Swederus, 1787) & $\begin{array}{l}\text { (PIU, SULL, TAL, PAI, MORR, SECH, AYA, HUA) } \\
\text { (BESII, BESc, DES, MAN, BSI, BHM) }\end{array}$ & Juárez-Noé \& González-Coronado (2018) \\
\hline \multicolumn{3}{|l|}{ Nemognatha Illiger, 1807} \\
\hline 228. Nemognatha chrysomeloides (Linnaeus, 1763) & (PIU) (BESII) & Juárez-Noé \& González-Coronado (2018) \\
\hline 229. Nemognatha sp. 1. & (PIU) (BESII) & Juárez-Noé \& González-Coronado (2018) \\
\hline \multicolumn{3}{|l|}{ Anthicidae Latreille, 1819} \\
\hline \multicolumn{3}{|l|}{ Anthicinae Latreille, 1819} \\
\hline \multicolumn{3}{|l|}{ Ischyropalpus Bonadona, 1896} \\
\hline 231. Ischyropalpus sericans (Erichson, 1834) & (PIU) (BESII) & Juárez-Noé \& González-Coronado (2018) \\
\hline \multicolumn{3}{|l|}{ Sapintus Casey, 1895} \\
\hline 232. Sapintus ovalis Werner, 1983 & $(\mathrm{SECH})(\mathrm{DES})$ & Juárez-Noé \& González-Coronado (2018) \\
\hline \multicolumn{3}{|l|}{ Vacusus Casey 1895} \\
\hline 233. Vacusus peruvianus $(\mathrm{Pic}, 1902)^{*}$ & (PIU) (BESII) & Juárez-Noé \& González-Coronado (2018) \\
\hline \multicolumn{3}{|l|}{ Anthicus Paykull, 1798} \\
\hline 234. Anthicus sp. 1. & (PIU) (BESII) & Juárez-Noé \& González-Coronado (2018) \\
\hline \multicolumn{3}{|l|}{ Notoxinae Stephens, 1829} \\
\hline \multicolumn{3}{|l|}{ Notoxus Geoffroy, 1762} \\
\hline 235. Notoxus peruvianus Pic, 1904 & (PIU) (BESII) & Juárez-Noé \& González-Coronado (2018) \\
\hline \multicolumn{3}{|l|}{ Plesionotoxus Geoffroy, 1762} \\
\hline 236. Plesionotoxus sp. 1. & (PIU) (BESII) & Juárez-Noé \& González-Coronado (2018) \\
\hline \multicolumn{3}{|l|}{ Cerambycidae Latreille, 1802} \\
\hline \multicolumn{3}{|l|}{ Prioninae Latreille, 1802} \\
\hline \multicolumn{3}{|l|}{ Prionacalus White, 1845} \\
\hline 237. Prionacalus cacicus (White, 1845) & (HUA, AYA) (BHM) & Juárez-Noé \& González-Coronado (2018) \\
\hline $\begin{array}{l}\text { 238. Prionacalus inermus Komiya \& Santos-Silva, } \\
2018^{*} \dagger\end{array}$ & (HUA) (BHM) & Komiya \& Santos-Silva (2018) \\
\hline \multicolumn{3}{|l|}{ Psalidognathus Gray, 1831} \\
\hline $\begin{array}{l}\text { 239. Psalidognathus antonkozlovi Noguchi \& } \\
\text { Santos-Silva, } 2016\end{array}$ & (HUA) (BHM) & Juárez-Noé \& González-Coronado (2018) \\
\hline \multicolumn{3}{|l|}{ Cerambycinae Latreille, 1802} \\
\hline \multicolumn{3}{|l|}{ Achryson Audinet-Serville, 1833} \\
\hline 240. Achryson lineolatum Erichson, 1847 & $\begin{array}{l}\text { (PIU, SULL, TAL, SECH, MORR) (BESII, BESc, } \\
\text { DES) }\end{array}$ & Juárez-Noé \& González-Coronado (2018) \\
\hline \multicolumn{3}{|l|}{ Neoclytus Thomson, 1860} \\
\hline 241. Neoclytus unicolor (Laporte \& Gory, 1836) & (PIU) (BESII) & Juárez-Noé \& González-Coronado (2018) \\
\hline \multicolumn{3}{|l|}{ Eburia Lacordaire, 1830} \\
\hline 242. Eburia (Eburia) pilosa (Erichson, 1834) & $\begin{array}{l}\text { (PIU, SULL, TAL, SECH, MORR, PAI, AYA, HUA) } \\
\text { (BESII, BESc, BESm, DES, BSI, BHM) }\end{array}$ & Juárez-Noé \& González-Coronado (2018) \\
\hline 243. Eburia rufobrunnea Perroud, 1855 & (PIU, TAL) (BESII, DES) & Juárez-Noé \& González-Coronado (2018) \\
\hline \multicolumn{3}{|l|}{ Heterachthes Newman, 1840} \\
\hline 244. Heterachthes pallidipennis (Thomson, 1865) & (PIU, TAL, SULL, PAI) (BESII, DES) & \\
\hline \multicolumn{3}{|l|}{ Asynapteron Martins, 1970} \\
\hline 245. Asynapteron inca (Martins, 1962) & $(\mathrm{HUA})(\mathrm{BHM})$ & Juárez-Noé \& González-Coronado (2018) \\
\hline \multicolumn{3}{|l|}{ Obrium Dejean, 1821} \\
\hline 246. Obrium circunflexum Martins \& Galileo 2004 & (PIU) (BESII) & \\
\hline \multicolumn{3}{|l|}{ Smodicum Haldeman, 1847} \\
\hline 247. Smodicum brunneum Thomson, 1878 & (PIU) (BESII) & \\
\hline Lamiinae Latreille, 1825 & & \\
\hline Nesozineus Linsley \& Chemsak, 1966 & & \\
\hline
\end{tabular}


Tabla 1 (continuación).

\begin{tabular}{|c|c|c|}
\hline Taxones & Distribución (provincia) (paisaje ecológico) & Referencia \\
\hline 248. Nesozineus peruanus Galileo \& Martins 2007 & (PIU) (BESII) & Juárez-Noé \& González-Coronado (2018) \\
\hline 249. Nesozineus probolus Galileo \& Martins, 1996 & (PIU) (BESII) & Juárez-Noé \& González-Coronado (2018) \\
\hline \multicolumn{3}{|l|}{ Bisaltes Thomson, 1860} \\
\hline $\begin{array}{l}\text { 250. Bisaltes (Bisaltes) fuscomarmoratus } \\
\text { Breuning, } 1966\end{array}$ & (AYA) (BHM) & \\
\hline \multicolumn{3}{|l|}{ Fredlanea Martins \& Galileo, 1996} \\
\hline 251. Fredlanea sp. 1. & (AYA) (BHM) & Juárez-Noé \& González-Coronado (2018) \\
\hline \multicolumn{3}{|l|}{ Taeniotes Audinet-Serville, 1835} \\
\hline 252. Taeniotes marmoratus Thomson, 1865 & (AYA) (BHM) & \\
\hline \multicolumn{3}{|l|}{ Grammopsoides Breuning, 1940} \\
\hline 253. Grammopsoides tenuicornis (Casey, 1913) & (PIU, MORR) (BESII) & Juárez-Noé \& González-Coronado (2018) \\
\hline \multicolumn{3}{|l|}{ Emphytoeciosoma Melzer, 1934} \\
\hline $\begin{array}{l}\text { 254. Emphytoeciosoma flava Martins, Santos- } \\
\text { Silva \& Galileo, } 2015^{*}\end{array}$ & (MORR) (BHM) & Juárez-Noé \& González-Coronado (2018) \\
\hline \multicolumn{3}{|l|}{ Desmiphora Audinet-Serville, 1835} \\
\hline $\begin{array}{l}\text { 255. Desmiphora (Desmiphora) xerophila Martins } \\
\text { \& Galileo, } 1995 \dagger\end{array}$ & (MORR) (BESc) & \\
\hline \multicolumn{3}{|l|}{ Estola Fairmaire-Germain, 1859} \\
\hline 256. Estola sp $\dagger$ & (PIU) (BESII) & \\
\hline \multicolumn{3}{|l|}{ Atrypanius Bates, 1864} \\
\hline 257. Atrypanius implexus (Erichson, 1847)† & (PIU) (BESII) & \\
\hline \multicolumn{3}{|l|}{ Chrysomelidae Latreille, 1802} \\
\hline \multicolumn{3}{|l|}{ Bruchinae Latreille, 1802} \\
\hline \multicolumn{3}{|l|}{ Amblycerus Thunberg, 1815} \\
\hline 258. Amblycerus piurae (Pierce, 1915) & $\begin{array}{l}\text { (PIU, SULL, TAL, PAI, SECH, MORR) (BESII, BESc, } \\
\text { DES) }\end{array}$ & Juárez-Noé \& González-Coronado (2018) \\
\hline \multicolumn{3}{|l|}{ Acanthoscelides Schilsky, 1905} \\
\hline 259. Acanthoscelides obtectus (Say, 1831) & $\begin{array}{l}\text { (PIU, SULL, TAL, SECH, MORR, PAI) (BESII, BESc, } \\
\text { DES) }\end{array}$ & Juárez-Noé \& González-Coronado (2018) \\
\hline $\begin{array}{l}\text { 260. Acanthoscelides suramerica Johnson, } \\
\text { 1990† }\end{array}$ & (PIU) (BESII) & \\
\hline \multicolumn{3}{|l|}{ Callosobruchus Pic, 1902} \\
\hline 261. Callosobruchus maculatus (Fabricius, 1775) & (PIU, SULL, TAL, SECH) (BESII, DES) & Juárez-Noé \& González-Coronado (2018) \\
\hline \multicolumn{3}{|l|}{ Megacerus Fahraeus, 1839} \\
\hline 262. Megacerus sp. 1. & (SULL) (BESII) & Juárez-Noé \& González-Coronado (2018) \\
\hline $\begin{array}{l}\text { 263. Megacerus (Megacerus) flabelliger } \\
\text { (Fahraeus, 1839) }\end{array}$ & (PIU) (BESII) & Juárez-Noé \& González-Coronado (2018) \\
\hline \multicolumn{3}{|l|}{ Sennius Bridwell, 1946} \\
\hline 264. Sennius peruanus (Pierce, 1915) & (PIU) (BESII) & Juárez-Noé \& González-Coronado (2018) \\
\hline \multicolumn{3}{|l|}{ Zabrotes Horn, 1885} \\
\hline 265. Zabrotes subfasciatus (Boheman, 1833)† & $(\mathrm{SECH})(\mathrm{BESII})$ & \\
\hline \multicolumn{3}{|l|}{ Criocerinae Latreille, 1804} \\
\hline \multicolumn{3}{|l|}{ Lema Fabricius, 1798} \\
\hline 266. Lema sp. 1. & (PIU) (BESII) & Juárez-Noé \& González-Coronado (2018) \\
\hline \multicolumn{3}{|l|}{ Plagiometriona Spaeth, 1899} \\
\hline 267. Plagiometriona steinheili (Wagener, 1877) & (AYA) (BHM) & Juárez-Noé \& González-Coronado (2018) \\
\hline \multicolumn{3}{|l|}{ Sceloenopla Chevrolat, 1837} \\
\hline 268. Sceloenopla sp. 1. & (AYA) (BHM) & Juárez-Noé \& González-Coronado (2018) \\
\hline \multicolumn{3}{|l|}{ Chrysomelinae Latreille, 1802} \\
\hline \multicolumn{3}{|l|}{ Desmogramma Erichson, 1847} \\
\hline 269. Desmogramma sp. 1. & (HUA) (BSI, BHM) & Juárez-Noé \& González-Coronado (2018) \\
\hline \multicolumn{3}{|l|}{ Chrysomela Linnaeus, 1758} \\
\hline 270. Chrysomela (Macrolina) scripta & (PIU) (BESII) & Juárez-Noé \& González-Coronado (2018) \\
\hline Galerucinae Latreille, 1802 & & \\
\hline
\end{tabular}


Tabla 1 (continuación).

\begin{tabular}{|c|c|c|}
\hline Taxones & Distribución (provincia) (paisaje ecológico) & Referencia \\
\hline \multicolumn{3}{|l|}{ Metalepta Baly, 1861} \\
\hline 271. Metalepta sp. 1. & (AYA) (BHM) & Juárez-Noé \& González-Coronado (2018) \\
\hline \multicolumn{3}{|l|}{ Cerotoma Chevrolat, 1843} \\
\hline 272. Cerotoma fascialis Erichson, 1847 & (PIU, SULL) (BESII) & Juárez-Noé \& González-Coronado (2018) \\
\hline \multicolumn{3}{|l|}{ Diabrotica Chevrolat, 1837} \\
\hline 273. Diabrotica speciosa Baly, 1886 & $\begin{array}{l}\text { (PIU, SULL, TAL, PAI, SECH, MORR, AYA, HUA) } \\
\text { (BESII, BESc, BESm, DES, MAN, BSI, BHM) }\end{array}$ & Juárez-Noé \& González-Coronado (2018) \\
\hline 274. Diabrotica septemliturata Erichson, 1847 & (PIU) (BESII) & Juárez-Noé \& González-Coronado (2018) \\
\hline 275. Diabrotica mauliki Barber, 1947 & (AYA) (BHM) & Juárez-Noé \& González-Coronado (2018) \\
\hline \multicolumn{3}{|l|}{ Chthoneis Baly, 1864} \\
\hline 276. Chthoneis sp. 1. & $(\mathrm{HUA})(\mathrm{BHM})$ & Juárez-Noé \& González-Coronado (2018) \\
\hline \multicolumn{3}{|l|}{ Exora Chevrolat, 1837} \\
\hline 277. Exora encaustica Bechyné, 1958 & (HUA) (BSI, BHM) & Juárez-Noé \& González-Coronado (2018) \\
\hline \multicolumn{3}{|l|}{ Cochabamba Bechyné, 1955} \\
\hline 278. Cochabamba chrysopleura (Harold, 1875)† & (HUA) (BHM) & Rocha (2010) \\
\hline \multicolumn{3}{|l|}{ Alticinae Latreille, 1802} \\
\hline \multicolumn{3}{|l|}{ Altica Fabricius, 1775} \\
\hline 279. Altica sp. 1. & (AYA) (BHM) & Juárez-Noé \& González-Coronado (2018) \\
\hline \multicolumn{3}{|l|}{ Disonycha Chevrolat, 1836} \\
\hline 280. Disonycha camposi Barber, 1936† & (PIU, SULL, TAL, PAI, SECH, MORR) (BESII) & \\
\hline \multicolumn{3}{|l|}{ Asphaera Chevrolat, 1843} \\
\hline 281. Asphaera albomarginata (Latreille, 1833) & (AYA) (BSI, BHM) & Juárez-Noé \& González-Coronado (2018) \\
\hline 282. Asphaera sp. 1. & (MORR) (BHM) & Juárez-Noé \& González-Coronado (2018) \\
\hline \multicolumn{3}{|l|}{ Parasyphraea Bechyné, 1959} \\
\hline 283. Parasyphraea sp† & (MORR) (BESc) & \\
\hline \multicolumn{3}{|l|}{ Aspicela Dejean, 1837} \\
\hline 284. Aspicela nigroviridis Guerin, 1855 & (AYA, HUA) (BSI, BHM) & Juárez-Noé \& González-Coronado (2018) \\
\hline \multicolumn{3}{|l|}{ Epitrix Foudras, 1860} \\
\hline 285. Epitrix sp. 1. & (PIU) (BESII) & Juárez-Noé \& González-Coronado (2018) \\
\hline \multicolumn{3}{|l|}{ Neodiphaulaca Bechyné \& Bechyné, 1975} \\
\hline 286. Neodiphaulaca sp. 1. & (HUA) (BHM) & Juárez-Noé \& González-Coronado (2018) \\
\hline \multicolumn{3}{|l|}{ Phenrica Bechyné, 1959} \\
\hline 287. Phenrica sp. 1. & (PIU) (BESII) & Juárez-Noé \& González-Coronado (2018) \\
\hline \multicolumn{3}{|l|}{ Cryptocephalinae Gyllenhal, 1813} \\
\hline \multicolumn{3}{|l|}{ Cryptocephalus Geoffroy, 1762} \\
\hline 288. Cryptocephalus sp. 1 & $\begin{array}{l}\text { (PIU, SULL, TAL, SECH, PAI, MORR) (BESII, BESc, } \\
\text { DES, MAN) }\end{array}$ & Juárez-Noé \& González-Coronado (2018) \\
\hline \multicolumn{3}{|l|}{ Pachybrachis Chevrolat, 1836} \\
\hline 289. Pachybrachis sp. 1. & (PIU) (BESII) & Juárez-Noé \& González-Coronado (2018) \\
\hline \multicolumn{3}{|l|}{ Coscinoptera Lacordaire, 1848} \\
\hline 290. Coscinoptera sp† & (PIU) (BESII) & \\
\hline \multicolumn{3}{|l|}{ Eumolpinae Hope, 1840} \\
\hline \multicolumn{3}{|l|}{ Typophorus Chevrolat, 1837} \\
\hline 291. Typophorus sp. 1 & (PIU, SULL) (BESII, DES) & Juárez-Noé \& González-Coronado (2018) \\
\hline Myochrous Dejean, 1837 & & \\
\hline 292. Myochrous geminus Blake, 1950 & (PIU) (BESII) & \\
\hline Anthribidae Billberg, 1820 & & \\
\hline Subfamilia Choraginae Kirby, 1819 & & \\
\hline Género Araecerus Schöenherr, 1823 & & \\
\hline 293. Araecerus fasciculatus (DeGeer, 1775)† & (HUA, AYA) (BESc, BHM) & Juárez-Noé \& González-Coronado (2018) \\
\hline Dryophthoridae Schönherr, 1833 & & \\
\hline Rhynchophorinae Schönherr, 1833 & & \\
\hline
\end{tabular}


Tabla 1 (continuación).

\begin{tabular}{|c|c|c|}
\hline Taxones & Distribución (provincia) (paisaje ecológico) & Referencia \\
\hline \multicolumn{3}{|l|}{ Rhynchophorus Herbst, 1795} \\
\hline 294. Rhynchophorus palmarum Linnaeus, 1758 & (PIU, SULL) (BESII) & Juárez-Noé \& González-Coronado (2018) \\
\hline \multicolumn{3}{|l|}{ Sphenophorus Schöenherr, 1838} \\
\hline 295. Sphenophorus sp. 1. & (PIU, SULL, TAL, PAI, SECH) (BESII) & Juárez-Noé \& González-Coronado (2018) \\
\hline \multicolumn{3}{|l|}{ Cosmopolites Chevrolat, 1885} \\
\hline 296. Cosmopolites sordidus Germar, 1824 & (PIU, SULL, TAL, PAI, SECH) (BESII) & Juárez-Noé \& González-Coronado (2018) \\
\hline \multicolumn{3}{|l|}{ Metamasius Horn, 1873} \\
\hline 297. Metamasius hemipterus (Linnaeus, 1758) & (PIU, SULL, TAL, PAI) (BESII) & \\
\hline \multicolumn{3}{|l|}{ Sitophilus Schöenherr, 1838} \\
\hline 298. Sitophilus zeamais Mostchulsky, 1855 & $\begin{array}{l}\text { (PIU, SULL, TAL, PAI, SECH, MORR, HUA, AYA) } \\
\text { (BESII, BESc, BSI, BHM) }\end{array}$ & Juárez-Noé \& González-Coronado (2018) \\
\hline 299. Sitophilus oryzae (Linnaeus, 1763) & $\begin{array}{l}\text { (PIU, SULL, TAL, PAI, SECH, MORR, HUA, AYA) } \\
\text { (BESII, BESc, BSI, BHM) }\end{array}$ & Juárez-Noé \& González-Coronado (2018) \\
\hline \multicolumn{3}{|l|}{ Curculionidae Latreille, 1802} \\
\hline \multicolumn{3}{|l|}{ Curculioninae Latreille, 1802} \\
\hline \multicolumn{3}{|l|}{ Anthonomus Germar, 1817} \\
\hline 300. Anthonomus vestitus Boheman, C.H., 1859 & (PIU, SULL, TAL. PAI) (BESII, BSI) & Juárez-Noé \& González-Coronado (2018) \\
\hline 301. Anthonomus sp. 1. & (PIU) (BESII) & Juárez-Noé \& González-Coronado (2018) \\
\hline \multicolumn{3}{|l|}{ Sibinia Germar, 1817} \\
\hline 302. Sibinia peruana Pierce, 1915 & (PIU) (BESII) & Juárez-Noé \& González-Coronado (2018) \\
\hline \multicolumn{3}{|l|}{ Conoderinae Schönherr, 1833} \\
\hline \multicolumn{3}{|l|}{ Copturus Schönherr, 1825} \\
\hline 303. Copturus aurivillianus Heller, 1895 & (SULL) (BESII) & Juárez-Noé \& González-Coronado (2018) \\
\hline \multicolumn{3}{|l|}{ Cyclominae Schönherr, 1826} \\
\hline \multicolumn{3}{|l|}{ Listroderes Schönherr, 1826} \\
\hline 304. Listroderes sp. 1. & $(\mathrm{TAL})(\mathrm{BESII})$ & Juárez-Noé \& González-Coronado (2018) \\
\hline \multicolumn{3}{|l|}{ Cryptorhynchinae Schönherr, 1825} \\
\hline \multicolumn{3}{|l|}{ Merocnemus Faust 1896} \\
\hline 305. Merocnemus binotatus Boheman, 1844 & (PIU) (BESII) & Juárez-Noé \& González-Coronado (2018) \\
\hline \multicolumn{3}{|l|}{ Eutinobothrus Faust, 1896} \\
\hline 306. Eutinobothrus gossypii (Pierce, 1915) & (PIU) (BESII) & Juárez-Noé \& González-Coronado (2018) \\
\hline \multicolumn{3}{|l|}{ Entiminae Schöenherr, 1823} \\
\hline \multicolumn{3}{|l|}{ Pandeleteius Schöenherr, 1834} \\
\hline 307. Pandeleteius variegatus (Pierce, 1915) & $\begin{array}{l}\text { (PIU, SULL, TAL, PAI, MORR, SECH, HUA) (BESII, } \\
\text { BESc, DES, BSI) }\end{array}$ & Juárez-Noé \& González-Coronado (2018) \\
\hline \multicolumn{3}{|l|}{ Plectrophoroides Wibmer \& O’Brien, 1986} \\
\hline 308. Plectrophoroides sp. 1. & (PIU) (BESII) & Juárez-Noé \& González-Coronado (2018) \\
\hline \multicolumn{3}{|l|}{ Eustylus Sahlberg, 1823} \\
\hline 309. Eustylus humilis (Erichson, 1847) & (PIU, SULL, PAI) (BESII) & \\
\hline \multicolumn{3}{|l|}{ Brachyomus Lacordaire, 1863} \\
\hline 310. Brachyomus sp $\dagger$ & (AYA) (BHM) & \\
\hline \multicolumn{3}{|l|}{ Naupactus Dejean, 1821} \\
\hline 311. Naupactus sp $\dagger$ & (PIU) (BESII) & \\
\hline Baridinae Schönherr, 1836 & & \\
\hline Linogeraeus Casey, 1920 & & \\
\hline 312. Linogeraeus perscitus (Herbst, 1797) & (PIU) (BESII) & Juárez-Noé \& González-Coronado (2018) \\
\hline Eurhinus Illiger, 1807 & & \\
\hline 313. Eurhinus festivus (Fabricius, 1792) & (MORR) (BESc) & \\
\hline Molytinae Schönherr, 1823 & & \\
\hline Conotrachelus Dejean, 1821 & & \\
\hline 314. Conotrachelus sp. 1. & (TAL, HUA) (BESII, BSI) & Juárez-Noé \& González-Coronado (2018) \\
\hline Rhyssomatus (Schönherr, 1837) & & \\
\hline 315. Rhyssomatus sp. 1. & (PIU) (BESII) & Juárez-Noé \& González-Coronado (2018) \\
\hline
\end{tabular}


Tabla 1 (continuación).

\begin{tabular}{|c|c|c|}
\hline Taxones & Distribución (provincia) (paisaje ecológico) & Referencia \\
\hline \multicolumn{3}{|l|}{ Scolytinae Latreille, 1804} \\
\hline \multicolumn{3}{|l|}{ Pagiocerus Eichhoff, 1868} \\
\hline 316. Pagiocerus frontalis (Fabricius, 1801) & (PIU) (BESII) & \\
\hline \multicolumn{3}{|l|}{ Hypothenemus Westwood, 1834} \\
\hline 317. Hypothenemus hampei (Ferrari, 1867) & $(\mathrm{HUA})(\mathrm{BHM})$ & \\
\hline
\end{tabular}

\section{Listado faunístico}

Suborden Adephaga

Familia Gyrinidae Latreille, 1810

Subfamilia Gyrininae Latreille, 1810

Género Andogyrus Ochs, 1924

1. Andogyrus peruvianus Régimbart, $1907 \dagger$

Material estudiado. 1 đ̆, Ayabaca, caserío Yacupampa, 0510'51"S, 80³7'31"O, 2750 msnm, 23-XI-2018, red acuática, U. González, Bosque Húmedo de montaña.

Comentarios. Primera especie de Gyrinidae para la región, característica de cuerpos de agua de alta montaña en los andes (Brinck, 1977; Bustamante, 2018).

Familia Carabidae Latreille, 1802

Subfamilia Carabinae Latreille, 1802

Género Calosoma Weber, 1801

2. Calosoma (Castrida) rufipenne Dejean, $1831 \uparrow$

Material examinado. 1 ej., Ayabaca, Paimas, 4³7'7’'S7956’43”O, 550 msnm, Bosque estacionalmente seco de colina.

Comentarios. Segunda especie de Calosoma para la región, anteriormente se citaba a Calosoma (Castrida) abbreviatum Chaudoir, 1869 (Juárez-Noé \& GonzálezCoronado, 2018). Ambas especies se distribuyen en bosques estacionalmente secos.

REFERENCIA: Gidaspow (1963).

Subfamilia Harpalinae Bonelli, 1810

Género Notiobia Perty, 1830

3. Notiobia (Anisotarsus) peruviana (Dejean, 1829)

Material examinado. 2 ふ̄̋̂, Piura, Castilla, Universidad Nacional de Piura, $05^{\circ} 10^{\prime} 51^{\prime \prime} \mathrm{S}-80^{\circ} 37^{\prime} 31^{\prime \prime} \mathrm{O}, 29 \mathrm{msnm}$, 12-IV-2016, trampas pitfall, U. González, Bosque estacionalmente seco de llanura, 005 CAR-GJN; 1 ô, Sullana, Marcavelica, 0452'54"S-8042'12"O, 40 msnm, 26-II-2018, trampas pitfall, G. Juárez, Bosque estacionalmente seco de llanura; 1 q, Sullana, Ignacio Escudero,

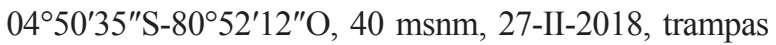
pitfall, G. Juárez, Bosque estacionalmente seco de lla-

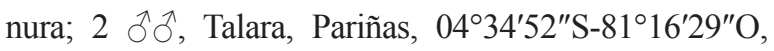
20 msnm, 14-VI-2018, trampas pitfall, G. Juárez, Bosque estacionalmente seco de llanura; $1 \hat{\jmath}$, Paita, Vichayal, 0451'55"S-81 04'19"O, 10 msnm, 17-VII-2018, trampas pitfall, G. Juárez, Bosque estacionalmente seco de llanura; 1 ㅇ, Sechura, Cristo nos Valga, 05²9'37"S-8044'28"O, 10 msnm, 25-VIII-2018, trampas pitfall, G. Juárez, Bosque estacionalmente seco de llanura; 2 우, Morropón, Buenos Aires, 0566'02"S-7958'03"O, 135 msnm, 12-VIII-2018, trampas pitfall, G. Juárez, Bosque estacionalmente seco de llanura.

Comentarios. Especie citada como Notiobia sp. 1 en Juárez-Noé \& González-Coronado (2018). Especie que amplía su distribución a las provincias de Sullana, Paita, Talara, Sechura, Morropón y se limita a bosques estacionalmente secos, anteriormente citada para la provincia de Piura (Juárez-Noé \& GonzálezCoronado, 2018).

Familia Dytiscidae Leach, 1815

Subfamilia Agabinae Thomson, 1867

Género Agametrus Sharp, 1882

Comentarios, Las especies de Agametrus son característicos de cuerpos de agua de alta montaña en los andes (Miller \& Bergsten, 2016).

\section{Agametrus monticola (Guignot, 1958) $†$}

Material examinado. 1 ej., Ayabaca, Ayabaca, centro poblado Huachuma, 0451'68'S-7976'41"O, 2700 msnm, Bosque húmedo de montaña.

REFERENCIA. Nilsson \& Hájek (2018).

\section{Agametrus andinus Guignot, $1958 \dagger$}

Material eXAminAdo. 1 ej., Ayabaca, 04³8'13"S-7943'26"O, 2700 msnm, Bosque húmedo de montaña.

REFERENCIA. Nilsson \& Hájek (2018). 
Subfamilia Copelatinae Branden, 1885

Género Copelatus Erichson, 1832

6. Copelatus blancasi Guignot, $1958 \dagger$

Material examinado. Ayabaca, centro poblado de

Huachuma, 0451'68's-7976'41"O, 2700 msnm,

Bosque húmedo de montaña.

REFERENCIA. Nilsson \& Hájek (2018).

Subfamilia Colymbetinae Erichson, 1837

Género Meridiorhantus Balke, Hájek \& Hendrich, 2017

7. Meridiorhantus calidus (Fabricius, 1792) $\dagger$

Material examinado. 1 †, Piura, Castilla, Universidad

Nacional de Piura, $05^{\circ} 10^{\prime} 51^{\prime \prime} \mathrm{S}-80^{\circ} 37^{\prime} 31^{\prime \prime} \mathrm{O}, 29 \mathrm{msnm}$,

2-II-2018, red acuática, U. González, Bosque estacionalmente seco de llanura; 1 , Sullana, Salitral, 0451'27"S-8040'52"O, 40 msnm, 6-IV-2016, red acuática, G. Juárez, Bosque estacionalmente seco de llanura; 1 o, Talara, Lobitos, $04^{\circ} 27^{\prime} 10^{\prime \prime} \mathrm{S}-81^{\circ} 16^{\prime} 40^{\prime \prime} \mathrm{O}$, 12 msnm, 27-VI-2014, trampas luz, G. Juárez, Desierto; 1 †, Paita, $05^{\circ} 04^{\prime} \mathrm{S}-81^{\circ} 06^{\prime} \mathrm{O}, 10 \mathrm{msnm}$, 7-IX-2013, trampa luz, G. Juárez, Bosque estacionalmente seco de llanura; 3 $\widehat{\partial}$, Paita, Vichayal, 04 51'55"S-81 04'19"O, 10 msnm, 20-X-2018, red acuática, G. Juárez, Manglar; 1 q, Sechura, Desierto de Sechura, 05 $34^{\prime}$ S-81 $11^{\circ} \mathrm{O}, 10$ msnm, 13-XII-2013, trampa luz, G. Juárez, Desierto; 1 ㅇ, Morropón, Piedra del Toro, $05^{\circ} 11^{\prime} \mathrm{S}-80^{\circ} 49^{\prime} \mathrm{O}, 1225 \mathrm{msnm}, 29-\mathrm{VII}-$ 2013, red acuática, G. Juárez, Bosque estacionalmente seco de colina; 1 đ̃, Huancabamba, Sondorillo, 05 27'07"S-79³6'22"O, 1600 msnm, 17-XI-2012, red acuática, G. Juárez, Bosque seco interandino; 2 우, Huancabamba, Canchaque, Cerro Huayanay, 05²4'07"S-79³6'22"O, 1600 msnm, 15-V-2018, trampa luz, G. Juárez, Bosque húmedo de montaña;

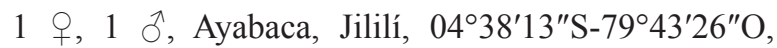
450 msnm, 16-XI-2013, red acuática, G. Juárez, Bosque seco interandino.

Subfamilia Laccophilinae Gistel, 1856

Género Laccophilus Leach, 1815

8. Laccophilus normifer Guignot, $1958 \dagger$

Material examinado. 1 ej., Ayabaca, centro poblado de Joras, 0453'33"S-79653'26"O, 2700 msnm, Bosque húmedo de montaña.

ReFerencia. Nilsson \& Hájek (2018).

Suborden Polyphaga

Familia Histeridae Gyllenhal, 1808

Subfamilia Saprinae Blanchard, 1845

Género Euspilotus Lewis, 1907
9. Euspilotus (Hesperosaprinus) erythropleurus (Marseul, $1855) \dagger$

Material examinado. 1 q, Sullana, $04^{\circ} 54^{\prime} \mathrm{S}-80^{\circ} 42^{\prime} \mathrm{O}, 30$ msnm, 24-II-2012, D. Saavedra, Bosque estacionalmente seco de llanura.

Comentarios. Primera especie de Euspilotus para la región, los individuos fueron colectados bajo excremento humano (Arriagada, 2015).

REFERENCIA. Arriagada (2015).

Género Hypocaccus Thomson, 1867

10. Hypocaccus (Baeckmanniolus) gaudens (LeConte, $1851) \dagger$

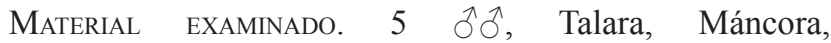
04은 $26^{\prime \prime S}-81^{\circ} 02^{\prime} 50^{\prime \prime} \mathrm{O}, 7$ msnm, 28-VIII-2010, V.M.

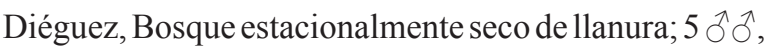
5 우오, Sechura, Chulliyachi, $05^{\circ} 33^{\prime} 27^{\prime \prime} \mathrm{S}-80^{\circ} 49^{\prime} 20^{\prime \prime} \mathrm{O}$, 6 msnm, 25-IV-2012, D. Saavedra, Desierto.

Comentarios. Primera especie de Hypocaccus para la región, los individuos fueron colectados bajo excremento humano y bajo peces muertos (Arriagada, 2015).

REFERENCIA. Arriagada (2015).

Familia Silphidae Latreille, 1806

Subfamilia Silphinae Latreille, 1806

Género Oxelytrum Gistel, 1848

11. Oxelytrum anticola (Guérin-Méneville, 1855) †

Material eXAminado. 1 , $1 \hat{\jmath}$, Huancabamba, Canchaque, Pampa Minas, 05²1'20.6"S-79³5'20"O, 1600 msnm, 11-V-2018, G. Juárez, Bosque húmedo de montaña.

Comentarios. Primera especie de Silphidae para la región. Los individuos estudiados fueron colectados en cadáveres de Sus scrofa domestica Linnaeus, 1758.

Familia Staphylinidae Latreille, 1802

Subfamilia Paederinae Fleming, 1821

Género Paederus Fabricius, 1775

12. Paederus ornaticornis Sharp, $1891 \dagger$

Material examinado. $4 \hat{\jmath} \widehat{\partial}$, Piura, Castilla, caserío Miraflores, $05^{\circ} 16^{\prime} 66^{\prime \prime} \mathrm{S}-80^{\circ} 61^{\prime} 67^{\prime \prime} \mathrm{O}, 29 \mathrm{msnm}, 12-\mathrm{IV}$ 2018, trampa luz, U. González, Bosque estacionalmente seco de llanura; 1 $\hat{\jmath}$, Piura, Tambogrande,

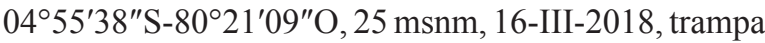
luz, G. Juárez, Bosque estacionalmente seco de llanura; 1 đ̊, Sullana, Marcavelica, 0452'54"S-8042'12"O, 40 msnm, 26-II-2018, trampa luz, G. Juárez, Bosque estacionalmente seco de llanura; 1 †, Sullana,

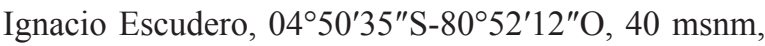
27-II-2018, trampa luz, G. Juárez, Bosque estacionalmente seco de llanura; $2 \hat{\jmath} \widehat{\jmath}$, Talara, Pariñas, 


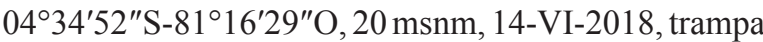
luz, G. Juárez, Bosque estacionalmente seco de lla-

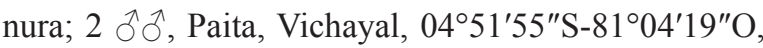
10 msnm, 17-VII-2018, trampa luz, G. Juárez, Bosque estacionalmente seco de llanura; 2 $q$, Sechura, Cristo nos Valga, $05^{\circ} 29^{\prime} 37^{\prime \prime} \mathrm{S}-80^{\circ} 44^{\prime} 28^{\prime \prime} \mathrm{O}, 10 \mathrm{msnm}, 25$-VIII2018, trampa luz, G. Juárez, Bosque estacionalmente seco de llanura; 2 우, Morropón, Santa Catalina de Mossa, 0506'10"S-79 53'06"O, 1000 msnm, 12-VIII2018, trampa luz, G. Juárez, Bosque seco interandino; 2 우, Huancabamba, Canchaque, Cerro Huayanay, 05²4'07"S-79³6'22"O, 1600 msnm, 15-V-2018, trampa luz, G. Juárez, Bosque húmedo de montaña;

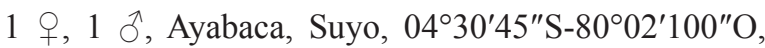
450 msnm, 5-V-2018, trampa luz, G. Juárez, Bosque estacionalmente seco de colina.

Comentarios. Especie de amplia distribución abarcando todas las provincias y paisajes ecológicos de la región. Todos los individuos estudiados fueron capturados a través de trampas de luz.

Subfamilia Staphylininae Latreille, 1802

Género Leptopeltus Bernhauer, 1906

13. Leptopeltus flavipennis (Erichson, 1840) $\dagger$

Material examinado. 1 ô, 1 q, Ayabaca, Bosque Ramos,

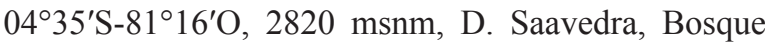
húmedo de montaña.

Referencia. Chani-Posse \& Asenjo (2013).

Género Plochionocerus Dejean, 1883

14. Plochionocerus humeralis (Sharp, 1885) $\dagger$

Material eXaminado. 1 ej., Sullana, Lancones, 04³8'27"S$80^{\circ} 32^{\prime} 55^{\prime \prime} \mathrm{O}, 650 \mathrm{msnm}$, Bosque estacionalmente seco de colina.

Referencia. Asiain et al. (2010).

Subfamilia Steninae MacLeay, 1825

Género Stenus Latreille, 1796

15. Stenus subcoeruleus Bernhauer, 1916†

Material EXAminado. $15 \hat{\jmath} \hat{\jmath}, 16$ 우, Huancabamba, Canchaque, $04^{\circ} 35^{\prime} \mathrm{S}-81^{\circ} 16^{\prime} \mathrm{O}, 1150 \mathrm{msnm}$, W. Markl, Bosque húmedo de montaña.

Referencia. Puthz (2017).

Subfamilia Aleocharinae Fleming, 1821

Género Rothium Moore \& Legner, 1977

16. Rothium evansi Ahn \& Ashe, 1996†

Material examinado. 1 ej., Piura, Paita, $05^{\circ} 04^{\prime} \mathrm{S}-81^{\circ} 06^{\prime} \mathrm{O}$,

$5 \mathrm{msnm}, \mathrm{G}$. Evans, Bosque estacionalmente seco de llanura.

ReFERENCIA. Ahn \& Ashe (1996)
Género Aleochara Gravenhorst, 1802

\section{Aleochara sp $\dagger$}

Material examinado. 1 \%, Piura, Paita, $05^{\circ} 04^{\prime} \mathrm{S}-81^{\circ} 06^{\prime} \mathrm{O}$, 5 msnm, 12-VIII-2018, colecta manual, G. Juárez, Bosque estacionalmente seco de llanura.

Familia Geotrupidae Latreille, 1802

Subfamilia Bolboceratinae Mulsant, 1842

Género Neoathyreus Howden \& Martinez, 1963

18. Neoathyreus (Neoathyreus) perryae Howden, 1985†

Material examinado. 1 đ, Talara, $04^{\circ} 35^{\prime} \mathrm{S}-81^{\circ} 16^{\prime} \mathrm{O}$, $15 \mathrm{msnm}$, Bosque estacionalmente seco de llanura.

Comentarios. Segunda especie de Geotrupidae para la región, anteriormente se citaba a Zefevazia peruana (Boucomont, 1902) (Juárez-Noé \& González-Coronado, 2018). Neoathyreus perryae no es citada para Perú por Ratcliffe et al. (2015), sin embargo Howden (1985) la registra para las regiones de Piura y La Libertad.

REFERENCIA. Howden (1985)

Familia Passalidae Leach, 1815

Género Veturius Kaup, 1871

19. Veturius (Veturius) standfussi Kuwert, $1891 \dagger$

Material examinado. 1 ej., Huancabamba, Abra de Porculla, 0549'60"S-79³0'O, 1900 msnm, Bosque húmedo de montaña.

Referencia. Salazar \& Boucher (2018).

20. Veturius (Ouayana) quaesitor Boucher \& Salazar, 2018 $\dagger^{*}$

Material examinado. $4 \hat{\jmath} \widehat{\jmath}, 2$ q $q$, Ayabaca, 0438'13"S$79^{\circ} 43^{\prime 2} 6^{\prime \prime} \mathrm{O}, 480 \mathrm{msnm}$, Bosque estacionalmente seco de colina.

Referencia. Salazar \& Boucher (2018).

Familia Lucanidae Latreille, 1804

Subfamilia Lucaninae Latreille, 1804

Género Incadorcus Arnaud \& Bomans, 2006

21. Incadorcus damasoi Arnaud \& Bomans 2006†*

Material examinado. 1 q, 12 $\widehat{\jmath}$, Ayabaca, Sícchez,

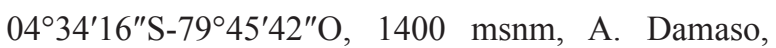
15-VIII-2006, Bosque estacionalmente seco de montaña.

ReFERENCIA. Arnaud \& Bomans (2006).

Género Aegognathus Leuthner, 1883

22. Aegognathus leuthneri damasoi Arnaud \& Bomans $2006 \div *$

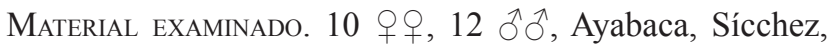

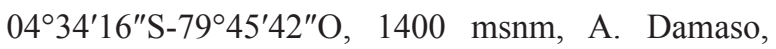
15-VIII-2006, Bosque estacionalmente seco de montaña. ReFERENCIA. Arnaud \& Bomans (2006). 
Género Sclerostomus Burmeister, 1847

23. Sclerostomus damasoi Arnaud \& Bomans 2006†*

Material examinado. 10 우, $10 \hat{\jmath} \widehat{\jmath}$, Ayabaca, Sícchez,

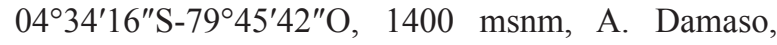
15-VIII-2006, Bosque estacionalmente seco de montaña.

REFERENCIA. Arnaud \& Bomans (2006).

\section{Sclerostomus wendyae Arnaud \& Bomans 2006†*}

Material examinado. 1 ej., Ayabaca, Sícchez, 04³4'16"S$79^{\circ} 45^{\prime} 42^{\prime \prime} \mathrm{O}$, Bosque estacionalmente seco de montaña.

ReFERENCIA. Coelho-Grossi (2011).

Familia Scarabaeidae Latreille, 1802

Género Onthophagus Latreille, 1802

25. Onthophagus confusus Boucomont, $1932 \dagger$

Material examinado. 4 ổ, 6 qo, Morropón, caserío Caracucho, $05^{\circ} 14^{\prime} 02^{\prime \prime} \mathrm{S}-79^{\circ} 91^{\prime} 56^{\prime \prime} \mathrm{O}, 560 \mathrm{msnm}$, 12-XII-2017, Bosque estacionalmente seco de colina.

Comentarios. Especie citada inicialmente como Onthophagus ophion confusus Boucomont, 1932 (Boucomont, 1932), sin embargo, Rossini et al. (2018) elevaron el nombre subespecífico a nivel de especie bajo el nuevo estado de Onthophagus confusus.

REFERENCIA. Rossini et al. (2018).

Familia Aphodiinae Leach, 1815

Género Aphodius Illiger, 1798

26. Aphodius (Labarrus) pseudolividus Balthasar, 1941

Material examinado. $4 \hat{\delta} \hat{\jmath}$, Huancabamba, Canchaque, 05²2'35"S-79³6'23"O, 2000 msnm, 5-V-2018, colecta manual, G. Juárez, Bosque húmedo de mon-

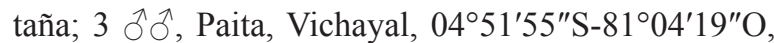
10 msnm, 20-X-2018, trampa luz, G. Juárez, Manglar.

Comentarios. Con estos registros la especie abarca ahora todas provincias y paisajes ecológicos de la región. Anteriormente estaba citada para las provincias de Piura, Sullana, Paita, Sechura, Talara, Morropón, Ayabaca y en los paisajes ecológicos de bosques estacionalmente secos, desierto, bosques secos interandinos (Juárez-Noé \& González-Coronado, 2018).

Subfamilia Rutelinae MacLeay, 1819

Género Platycoelia Dejean, 1833

27. Platycoelia marginata Burmeister, 1844

Material examinado. 1 $\hat{\jmath}$, Huancabamba, Canchaque, 05²2'35"S-79³6'23"O, 2000 msnm, 15-V-2017, colecta manual, G. Juárez, Bosque húmedo de montaña,
041 SCA-GJN; 1 q, Ayabaca, caserío Socchabamba, 0436'35"S-7942'34"O, 2335 msnm, 6-VIII-2017, trampa luz, G. Juárez, Bosque húmedo de montaña, 042 SCA-GJN.

Comentarios. Especie citada como Platycoelia sp. 1 en Juárez-Noé \& González-Coronado (2018). Hasta el momento la especie se distribuye en bosques húmedos de montaña.

Género Mecopelidnota Bates, 1904

28. Mecopelidnota arrowi Bates, 1904†

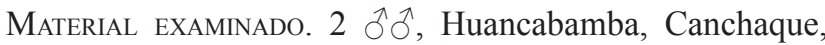
Pampa Minas, 05²1'20.6"S-79³5'20"O, 2100 msnm, 17-XI-2018, trampa luz, G. Juárez, Bosque húmedo de montaña.

Comentarios. Tercera especie de Mecopelidnota para la región, anteriormente se citaban a Mecopelidnota marxi Soula, 2008 y Mecopelidnota mezai Soula, 2008 (Juárez-Noé \& González-Coronado, 2018). Mecopelidnota marxi se distribuye en bosques estacionalmente secos mientras que $M$. mezai en bosques secos interandinos y bosques húmedos de montaña (Juárez-Noé \& González-Coronado, 2018).

Género Microrutela Bates, 1904

29. Microrutela campa (Ohaus, 1922) $\dagger$

Material examinado. 1 ej., Ayabaca, Suyo, Quiroz, $04^{\circ} 30^{\prime} 45^{\prime \prime} \mathrm{S}-80^{\circ} 02^{\prime} 100^{\prime \prime} \mathrm{O}, 450 \mathrm{msnm}$, Bosque estacionalmente de colina.

REFERENCIA. Jameson (1997).

Género Thyriochlorata Ohaus, 1915

30. Thyriochlorata villosa (Ohaus, 1908) †*

Material eXAminado. 1 ej., Huancabamba, 0514'22"S$79^{\circ} 26^{\prime} 59^{\prime \prime} \mathrm{O}, 3000$ msnm, Bosque húmedo de montaña. REFERENCIA. Soula (2002).

Género Chrysophora Dejean, 1821

31. Chrysophora chrysochlora (Latreille, 1811) $\dagger$

Material exAminado. 1 \%, Ayabaca, Ayabaca, Yacupampa, 0510'51"S-80³7'31"O, 2750 msnm, 17-XI-2018, trampa luz, G. Juárez, Bosque húmedo de montaña.

Subfamilia Dynastinae MacLeay, 1819

Género Cyclocephala Dejean, 1821

32. Cyclocephala macrophylla Erichson, 1847†

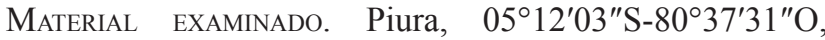
Bosque húmedo de montaña.

REFERENCIA. Moore et al. (2018). 
Género Tomarus Erichson, 1847

33. Tomarus peruvianus (Endrödi, 1970) (Fig. 1)

Material eXAminado. 1 q, Piura, Castilla, Universidad Nacional de Piura, 05¹0'51"S-80³7'31"O, 29 msnm, 2-II-2015, colecta manual, U. González, Bosque estacionalmente seco de llanura, 031 SCA-GJN; 1 , Sullana, Salitral, 04 $51^{\prime} 27^{\prime \prime} \mathrm{S}-80^{\circ} 40^{\prime} 52^{\prime \prime} \mathrm{O}, 40 \mathrm{msnm}$, 6-IV-2016, colecta manual, G. Juárez, Bosque estacionalmente seco de llanura. 045 SCA-GJN; 1 \%, Sullana, Querecotillo, 0450'24"S-80³8'57"O, 40 msnm, 12-VI-2016, colecta manual, G. Juárez, Bosque estacionalmente seco de llanura, 053 SCA-GJN; 1 q, Talara, Lobitos, $04^{\circ} 27^{\prime} 10^{\prime \prime} \mathrm{S}-81^{\circ} 16^{\prime} 40^{\prime \prime} \mathrm{O}, 12 \mathrm{msnm}, 27-\mathrm{VI}-$ 2014, trampa luz, G. Juárez, Desierto, 028 SCA-GJN; 1 \%, Paita, $05^{\circ} 04^{\prime} \mathrm{S}-81^{\circ} 06^{\prime} \mathrm{O}, 10 \mathrm{msnm}, 7-\mathrm{IX}-2013$, colecta manual, G. Juárez, Bosque estacionalmente seco de llanura, 017 SCA-GJN; 1 9, Sechura, Desierto de Sechura, $05^{\circ} 34^{\prime} \mathrm{S}-81^{\circ} 16^{\prime} \mathrm{O}, 10 \mathrm{msnm}, 13-\mathrm{XII}-2013$, colecta manual, G. Juárez, Desierto, 020 SCA-GJN; 1 †, Morropón, Piedra del Toro, $05^{\circ} 11^{\prime} \mathrm{S}-80^{\circ} 49^{\prime} \mathrm{O}$,
1225 msnm, 29-VII-2013, trampa luz, G. Juárez, Bosque estacionalmente seco de colina, 014 SCA-

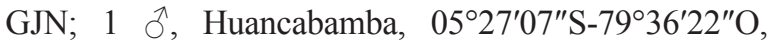
1600 msnm, 17-XI-2012, G. Juárez, Bosque seco interandino, 010 SCA-GJN; 1 †, 1 ô, Ayabaca, Suyo, 0430'45"S-8002'10"O, 450 msnm, 15-XI-2013, trampa pitfall, G. Juárez, Bosque estacionalmente seco de colina 018 SCA-GJN, 019 SCA-GJN.

Comentarios. Especie citada como Tomarus sp. 1 en JuárezNoé \& González-Coronado (2018). La especie se distribuye en todas las provincias de la región y se limita a ecosistemas secos: desierto y bosques estacionalmente secos.

\section{Género Golofa Hope, 1837}

\section{Golofa aegeon (Drury, 1773) $\dagger$}

Material examinado. $3 \hat{\jmath} \widehat{o}^{\lambda}$, Huancabamba, Canchaque, Pampa Minas, 05²1'20.6"S-79³5'20"O, 2100 msnm, 17-XI-2018, colecta manual, trampas pitfall, trampa luz, G. Juárez, Bosque húmedo de montaña.
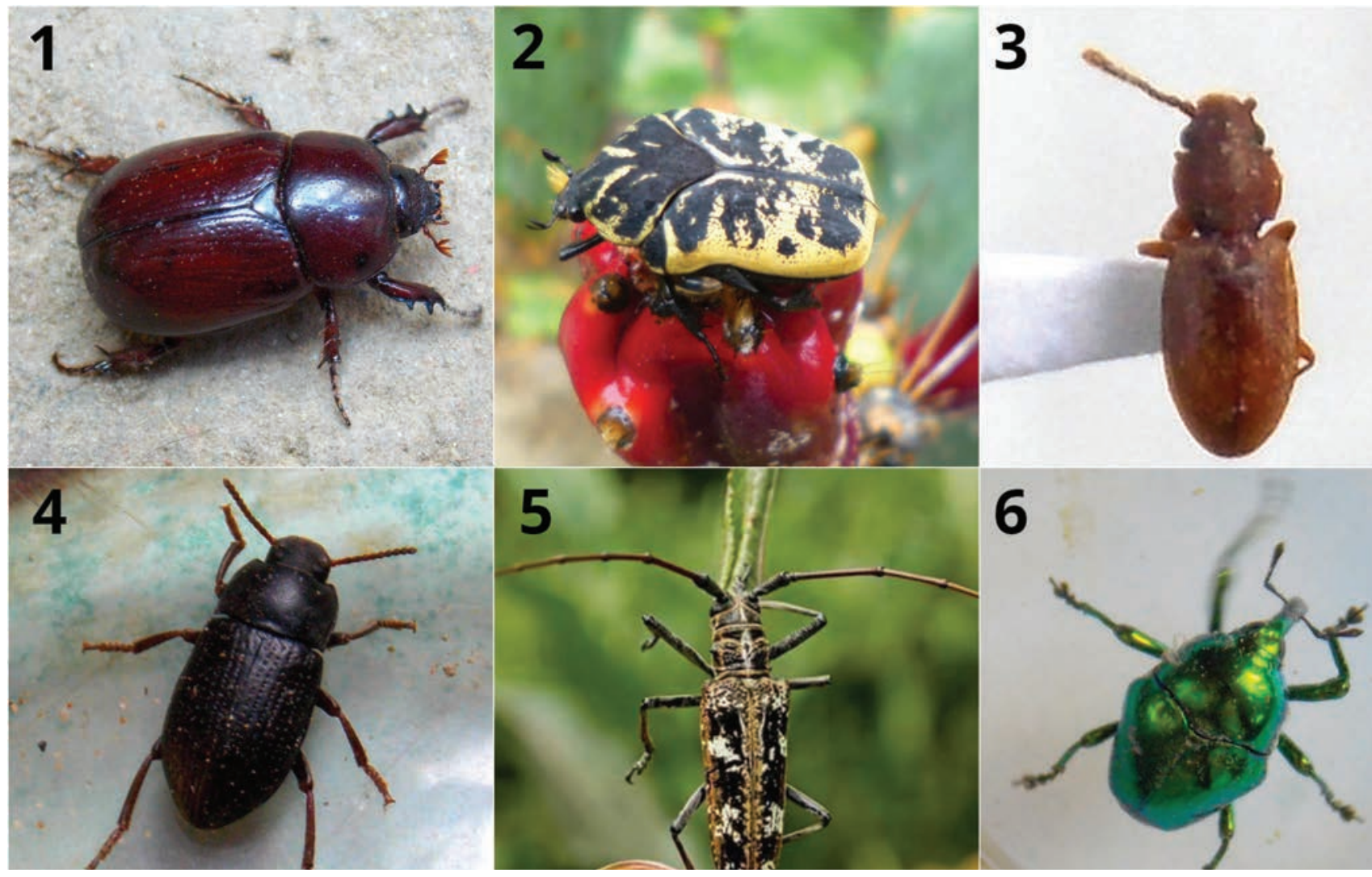

Figs. 1-6.- Habitus. 1. Tomarus peruvianus (Endrödi, 1970). 2. Gymnetis merops Ratcliffe, 2018. 3. Ahasverus advena (Waltl, 1834 ). 4. Alaetrinus pullus (Sahlberg, 1823). 5. Taeniotes marmoratus Thomson, 1865. 6. Eurhinus festivus (Fabricius, 1792). Fotografías 1-4, 6 de G. Juárez y U. González; fotografía 5 de l. Saldaña.

Figs. 1-6.- Habitus. 1. Tomarus peruvianus (Endrödi, 1970). 2. Gymnetis merops Ratcliffe, 2018. 3. Ahasverus advena (Waltl, 1834). 4. Alaetrinus pullus (Sahlberg, 1823). 5. Taeniotes marmoratus Thomson, 1865. 6. Eurhinus festivus (Fabricius, 1792). Photographs 1-4, 6 by G. Juárez and U. González; photograph 5 by I. Saldaña. 
Comentarios. Tercera especie de Golofa para la región, anteriormente se citaban a Golofa eacus Burmeister, 1847 y Golofa limogesi Ratcliffe \& Le Tirant, 2018 (Juárez-Noé \& González-Coronado, 2018). Las tres especies se distribuyen en bosques secos interandinos y bosques húmedos de montaña (Juárez-Noé \& González-Coronado, 2018).

Género Dynastes Kirby, 1825

35. Dynastes neptunus (Quensel, 1806)†

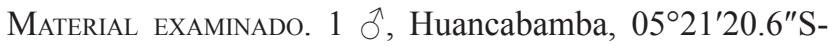
79³5'20"O, 2900 msnm, 17-V-2018, colecta manual, G. Juárez, Bosque húmedo de montaña.

Género Megasoma Kirby, 1825

36. Megasoma actaeon (Linnaeus, 1758) $\dagger$

Material examinado. 1 $\widehat{\jmath}$, Morropón, Chalaco, Bosque de Mijal, 0502'28'S-7947'45"O, 3000 msnm, 12-VII-2018, colecta manual, G. Juárez, Bosque húmedo de montaña.

Género Strategus Kirby, 1828

37. Strategus aloeus (Linnaeus, 1758)

Material eXaminado. 1 q, Huancabamba, San Miguel de El Faique, $05^{\circ} 24^{\prime} 07^{\prime \prime S}-79^{\circ} 36^{\prime} 22^{\prime \prime} \mathrm{O}, 1800 \mathrm{msnm}, 07-X I I-2018$, colecta manual, U. González, Bosque húmedo de montaña.

Comentarios. Especie que amplía su distribución a la provincia de Huancabamba, anteriormente citada para las provincias de Ayabaca y Morropón (Juárez-Noé \& González-Coronado, 2018).

Subfamilia Cetoniinae Leach, 1815

Género Gymnetis MacLeay, 1819

38. Gymnetis merops Ratcliffe, 2018* (Fig. 2)

Material examinado. 5 $\widehat{\partial}$, Morropón, Buenos Aires, 056'02"S-7958'03"O, 135 msnm, 12-III-2018, colecta manual, G. Juárez, Bosque estacionalmente seco de colina.

Comentarios. Con la reciente revisión del género Gymnetis realizada por Ratcliffe (2018) se llegó a la conclusión de que la especie citada como Gymnetis chevrolati balzarica Janson, 1880 en Juárez-Noé \& GonzálezCoronado (2018) se trata de G. merops. Con los nuevos ejemplares capturados esta especie amplía su distribución a la provincia de Morropón ya que anteriormente se citaba para las provincias de Piura, Sullana, Talara, Paita y Sechura (Juárez-Noé \& González-Coronado, 2018), limitándose a bosques estacionalmente secos. Los individuos estudiados fueron colectados sobre fruto de Opuntia ficus indica (L.) Mill., 1768 (Cactaceae).
Género Marmarina Kirby, 1827

39. Marmarina maculosa (Olivier, 1789) $\dagger$

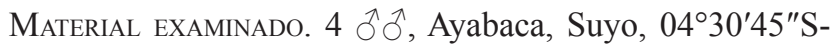
$80^{\circ} 02^{\prime} 100^{\prime \prime} \mathrm{O}, 450 \mathrm{msnm}, 5-\mathrm{V}-2018$, Bosque estacionalmente seco de colina.

REFERENCIA. Ratcliffe (2015).

Familia Buprestidae Leach, 1815

Subfamilia Buprestinae Lacordaire, 1857

Género Lasionota Mannerheim, 1837

40. Lasionota (Nelsonozodes) piurae Moore \& Diéguez, $2019 * \dagger$

Material examinado. 1 , Piura, 0516 $66^{\prime \prime} \mathrm{S}-80^{\circ} 61^{\prime} 67^{\prime \prime} \mathrm{O}$, 7-VI-1936, Wilcam, Bosque estacionalmente seco de llanura.

ReFerencia. Moore \& Diéguez (2019).

Familia Elateridae Leach, 1815

Subfamilia Agrypninae Candèze, 1857

Género Chalcolepidius Eschscholtz, 1829

41. Chalcolepidius corpulentus Candèze, $1874 \dagger$

Material eXAminado. 1 $\lesssim$, Talara, Negritos, 04³9'16"S$81^{\circ} 18^{\prime} 44^{\prime \prime} \mathrm{O}, 20 \mathrm{msnm}$, Bosque estacionalmente seco de llanura.

Comentarios. Segunda especie de Chalcolepidius para la región, anteriormente se citaba a Chalcolepidius fryi Candèze, 1874 (Juárez-Noé \& González-Coronado, 2018). Ambas especies se distribuyen en ecosistemas secos: desierto y bosques estacionalmente secos.

REFERENCIA. Casari (2002).

Género Conoderus Eschscholtz, 1829

42. Conoderus repandus (Erichson, 1847) $\dagger$

Material eXAminado. 1 $\widehat{\jmath}$, Piura, Castilla, caserío Miraflores, $05^{\circ} 16^{\prime} 66^{\prime \prime} \mathrm{S}-80^{\circ} 61^{\prime} 67^{\prime \prime} \mathrm{O}, 29 \mathrm{msnm}, 12-\mathrm{IV}$ 2018, colecta manual, G. Juárez, Bosque estacionalmente seco de llanura.

Comentarios. Primera especie de Conoderus para la región, Juárez-Noé \& González-Coronado (2018) citan a las morfoespecies Conoderus sp. 1 y Conoderus sp. 2.

Familia Cantharidae Imhoff, 1856 (1815)

Subfamilia Silinae Mulsant, 1862

Género Silis Charpentier, 1825

43. Silis foveolata Kirsch, $1865 \dagger$

Material eXAminado. 1 ô, Sullana, Mallares, 0452'S8046'O 29 msnm, 10-VI-1957, W. Markl, Bosque estacionalmente seco de llanura.

ReFEREncia. Constantin (2011). 
Familia Dermestidae Latreille, 1804

Subfamilia Dermestinae Latreille, 1804

Género Dermestes Linnaeus, 1758

44. Dermestes (Dermestinus) maculatus DeGeer, $1774 \dagger$

Material eXAminado. $2 \precsim \widehat{\jmath}$, Piura, Castilla, caserío Miraflores, 05 $16^{\prime} 66^{\prime \prime} \mathrm{S}-80^{\circ} 61^{\prime} 67^{\prime \prime} \mathrm{O}, 29 \mathrm{msnm}, 12-\mathrm{X}-2018$, colecta manual, G. Juárez, Bosque estacionalmente seco de llanura.

Comentarios. Los individuos estudiados fueron colectados sobre cuero de piel seca de Equus ferus caballus Linnaeus, 1758.

45. Dermestes (Dermestinus) frischii Kugellan, $1792 \dagger$

Material examinado. $1 \hat{\jmath}$, Piura, Castilla, caserío Miraflores, $05^{\circ} 16^{\prime} 66^{\prime \prime} \mathrm{S}-80^{\circ} 61^{\prime} 67^{\prime \prime} \mathrm{O}, 29 \mathrm{msnm}, 12-\mathrm{II}-$ 2019, colecta manual, G. Juárez, Bosque estacionalmente seco de llanura.

Comentarios. El individuo estudiado fue colectado en cadáver de cerdo en descomposición.

\section{Dermestes (Dermestes) ater DeGeer, 1774†}

Material eXAminado. 1 d, Piura, Castilla, caserío Miraflores, $05^{\circ} 16^{\prime} 66^{\prime \prime} \mathrm{S}-80^{\circ} 61^{\prime} 67^{\prime \prime} \mathrm{O}, 29 \mathrm{msnm}, 12-\mathrm{II}-$ 2019, colecta manual, G. Juárez, Bosque estacionalmente seco de llanura.

Comentarios. El individuo estudiado fue colectado en cadáver de cerdo en descomposición.

Familia Bostrichidae Latreille, 1802

Subfamilia Bostrichinae Latreille, 1802

Género Sinoxylon Duftschmid, 1825

47. Sinoxylon anale Lesne, 1897

Material examinado. $2 \hat{\jmath}$, Morropón, Buenos Aires, 05 66'02"S-79 58'03"O, 135 msnm, 12-XII-2018, colecta manual, U. González, Bosque estacionalmente seco de colina.

Comentarios. Especie que amplía su distribución a la provincia de Morropón y que se limita a bosques estacionalmente secos, anteriormente citada para la provincia de Piura (Juárez-Noé \& González-Coronado, 2018). Los individuos estudiados fueron colectados en el interior de troncos de Prosopis pallida (Humboldt \& Bonplant ex Willdenow), Kunth, 1823 (Fabaceae).

Familia Ptinidae Latreille, 1802

Subfamilia Anobiinae Kirby, 1837

Género Stegobium Motschulsky, 1860

48. Stegobium paniceum (Linnaeus, 1758) $\dagger$

Material examinado. $2 \hat{\jmath} \hat{\jmath}$, Piura, Castilla, caserío Miraflores, $05^{\circ} 16^{\prime} 66^{\prime \prime} \mathrm{S}-80^{\circ} 61^{\prime} 67^{\prime \prime} \mathrm{O}, 29 \mathrm{msnm}$,
10-XII-2018, colecta manual, G. Juárez, Bosque estacionalmente seco de llanura.

Comentarios. Especie considerada como plaga de productos almacenados (Hagstrum et al., 2013). Los individuos estudiados fueron colectados en granos almacenados de Triticum aestivum L., 1753 (Poaceae).

Familia Cleridae Latreille, 1802

Subfamilia Peloniinae Opitz, 2010

Género Pelonium Spinola, 1844

49. Pelonium riveti Lesne, $1909 \dagger$

Material examinado. 1 q, Paita, $05^{\circ} 04^{\prime} \mathrm{S}-81^{\circ} 06^{\prime} \mathrm{O}$, 10 msnm, 7-II-2019, colecta manual, G. Juárez, Desierto.

\section{Pelonium anteguttatum Opitz, 2018†}

Material examinado. 1 q, Sullana, Mallares, $37 \mathrm{msnm}$, 2-II-1956, W. Mark1, Bosque estacionalmente seco de llanura.

ReFERENCIA. Opitz (2018)

Familia Melyridae Leach, 1815

Subfamilia Melyrinae Leach, 1815

Género Astylus Laporte de Castelnau, 1836

51. Astylus bonplandi Erichson, $1847 \dagger$

Material eXaminado. 1 đ, Huancabamba, $05^{\circ} 02^{\prime} \mathrm{S}-79^{\circ} 04^{\prime} \mathrm{O}$, 3000 msnm, Bosque húmedo de montaña.

Comentarios. Tercera especie de Astylus para la región, anteriormente se citaban a Astylus longulus Constantin, 2011 y Astylus lojaensis Constantin, 2011 (Juárez-Noé \& González-Coronado, 2018). Todas las especies están distribuidas en bosques húmedos de montaña.

ReFERENCIA. Constantin (2011).

Familia Silvanidae Kirby, 1837

Subfamilia Silvaninae Kirby, 1837

Género Ahasverus Gozis, 1881

52. Ahasverus advena (Waltl, 1834)† (Fig. 3)

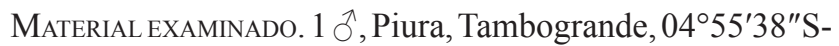
$80^{\circ} 21^{\prime} 09^{\prime \prime} \mathrm{O}, 25 \mathrm{msnm}, 14-\mathrm{III}-2018$, colecta manual, G. Juárez, Bosque estacionalmente seco de llanura.

Comentarios. Nuevo registro para Perú. Especie de distribución cosmopolita, considerada plaga de productos almacenados (Hagstrum et al., 2013), había sido registrado para Sudamérica pero aún no en Perú (Thomas \& Chaboo, 2015). El individuo estudiado fue colectado en granos almacenados de Phaseolus vulgaris L., 1753 infestado por hongos. 
Familia Coccinellidae Latreille, 1807

Subfamilia Coccinellinae Latreille, 1807

Género Delphastus Casey, 1899

53. Delphastus berryi Gordon, 1994†

MaterialeXAminado. $1 \delta^{\Uparrow}$, Sullana, Marcavelica, 0452'54"S$80^{\circ} 42^{\prime} 12^{\prime \prime} \mathrm{O}, 40 \mathrm{msnm}, 26-\mathrm{VII}-2018$, colecta manual, G. Juárez, Bosque estacionalmente seco de llanura;

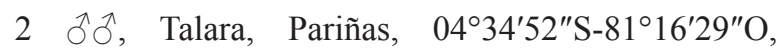
20 msnm, 11-X-2018, colecta manual, G. Juárez, Bosque estacionalmente seco de llanura; $1 \hat{\jmath}$, Paita, Vichayal, 04 $51^{\prime} 55^{\prime \prime S}-81^{\circ} 04^{\prime} 19^{\prime \prime} \mathrm{O}, 10 \mathrm{msnm}, 20-\mathrm{X}$ 2018, colecta manual, G. Juárez, Bosque estacionalmente seco de llanura.

Género Azya Mulsant, 1850

54. Azya orbigera ecuadorica Gordon, 1980

Materialexaminado. 10 , Sullana, Marcavelica, 04 $52^{\prime} 54^{\prime \prime}$ S$80^{\circ} 42^{\prime} 12^{\prime \prime} \mathrm{O}, 40 \mathrm{msnm}, 26-\mathrm{VII}-2018$, colecta manual, G. Juárez, Bosque estacionalmente seco de llanura;

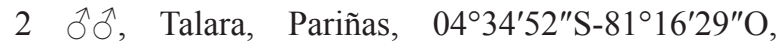
20 msnm, 11-X-2018, colecta manual, G. Juárez, Bosque estacionalmente seco de llanura; $1 \hat{\jmath}$, Paita, Vichayal, $04^{\circ} 51^{\prime} 55^{\prime \prime} \mathrm{S}-81^{\circ} 04^{\prime} 19^{\prime \prime} \mathrm{O}, 10 \mathrm{msnm}, 20-\mathrm{X}$ 2018, colecta manual, G. Juárez, Bosque estacionalmente seco de llanura.

COMEnTARios. Especie que amplía su distribución a las provincias de Sullana, Talara y Paita y que se limita a los bosques estacionalmente secos. Anteriormente citado para la provincia Piura (Juárez-Noé \& GonzálezCoronado, 2018). Los individuos examinados fueron colectados depredando Coccus sp (Coccidae).

Género Pentilia Mulsant, 1850

\section{Pentilia $\mathrm{sp}$}

Material examinado. $1 \hat{\jmath}$, Piura, Castilla, caserío Miraflores, $05^{\circ} 16^{\prime} 66^{\prime \prime} \mathrm{S}-80^{\circ} 61^{\prime} 67^{\prime \prime} \mathrm{O}, 29 \mathrm{msnm}, 21-\mathrm{X}-2018$, colecta manual, G. Juárez, Bosque estacionalmente seco de

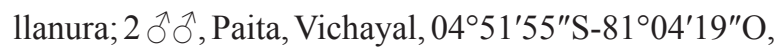
10 msnm, 7-II-2018, colecta manual, G. Juárez, Bosque estacionalmente seco de llanura; $2 \hat{\jmath} \hat{\jmath}$, Morropón, Buenos Aires, $05^{\circ} 66^{\prime} 02^{\prime \prime S}-79^{\circ} 58^{\prime} 03^{\prime \prime} \mathrm{O}, 135 \mathrm{msnm}$, 27-XI-2018, colecta manual, U. González, Bosque estacionalmente seco de colina.

Comentarios. En Juárez-Noé \& González-Coronado (2018) se menciona como Pentilia insidiosa Mulsant, 1850. En una reciente revisión de ejemplares se trataría de una especie no descrita (González, 2007). Amplía su distribución a las provincias de Piura, Paita y Morropón y se limita a los bosques estacionalmente secos. Anteriormente citado para la provincia
Sullana (Juárez-Noé \& González-Coronado, 2018). Los individuos estudiados fueron colectados depredando Pinnaspis aspidistrae Signoret (Diaspididae) en Persea americana Mill. (Lauraceae).

Género Brachiacantha Dejean, 1837

56. Brachiacantha darlene Gordon \& Canepari, 2014

Material examinado. 1 ô, Piura, Catacaos, 05²15'55"S$80^{\circ} 40^{\prime} 30^{\prime \prime}$. 29 msnm, 4-I-2018, colecta manual, G. Juárez, Bosque estacionalmente seco de llanura;

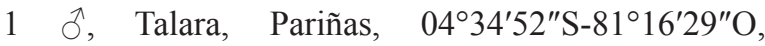
20 msnm, 4-VI-2018, colecta manual, G. Juárez, Bosque estacionalmente seco de llanura; 1 \&, Sechura, Cristo nos Valga, $05^{\circ} 29^{\prime} 37^{\prime \prime} \mathrm{S}-80^{\circ} 44^{\prime} 28^{\prime \prime} \mathrm{O}, 10 \mathrm{msnm}$, 2-XI-2018, colecta manual, G. Juárez, Bosque estacionalmente seco de llanura; $2 \hat{\jmath}$, Morropón, Buenos Aires, 0566'02"S-7958'03"O, 135 msnm, 8-XI-2018, colecta manual, G. Juárez, Bosque estacionalmente seco de colina.

COMEnTARios. Especie que amplía su distribución a las provincias de Talara, Sechura y Morropón y que se limita a los bosques estacionalmente secos. Anteriormente citado para las provincias de Piura y Sullana (JuárezNoé \& González-Coronado, 2018). Los individuos estudiados fueron colectados en Capparis avicennifolia Kunth y Colicodendron scabridum (Kunth) Hutchinson, 1852 (Capparaceae).

\section{Género Scymnobius Casey, 1899}

57. Scymnobius ecuadoricus Gordon \& González, 2005† Material examinado. $1 \widehat{\delta}$, Morropón, Caracucho, $05^{\circ} 14^{\prime} 02^{\prime \prime} \mathrm{S}$ 7991'56"O, 650 msnm, 17-VIII-2018, colecta manual, U. González, Bosque estacionalmente seco colina.

Comentarios. Nuevo registro para la región Piura, anteriormente citado solamente para la región Tumbes (Gordon \& González, 2005). Tercera especie de Scymnobius para la región, anteriormente se citaban a Scymnobius galapagoensis (Waterhouse, 1845) y Scymnobius triangularis Gordon \& González, 2002 (Juárez-Noé \& González-Coronado, 2018).

\section{Género Coleomegilla Timberlake, 1920}

58. Coleomegilla maculata bisexpunctata (Latreille, 1833) Material eXAminado. 1 q, Sullana, Ignacio Escudero,

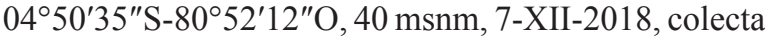
manual, G. Juárez, Bosque estacionalmente seco de

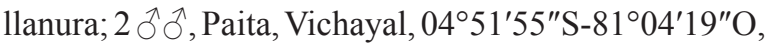
10 msnm, 3-IV-2018, colecta manual, G. Juárez, Bosque estacionalmente seco de llanura. 
Comentarios. Especie que amplía su distribución a las provincias de Sullana y Paita y que se limita a los bosques estacionalmente secos. Anteriormente citado para la provincia de Piura (Juárez-Noé \& González-Coronado, 2018). Los individuos estudiados fueron colectados en Acacia macracantha Humboldt \& Bonplant ex Willdenow, 1806 (Fabaceae) y Zea mays L. (Poaceae)

\section{Género Harmonia Mulsant, 1846}

\section{Harmonia axyridis (Pallas, 1772)}

Material eXaminado. 1 q, Sullana, Ignacio Escudero, 0450'35"S-8052'12"O, 40 msnm, 7-II-2018, colecta manual, G. Juárez, Bosque estacionalmente seco de

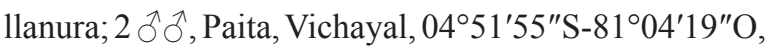
10 msnm, 3-IV-2018, colecta manual, G. Juárez, Bosque estacionalmente seco de llanura; $1 \hat{\jmath}$, Morropón, Buenos Aires, 0566'02"S-7958'03"O, 135 msnm, 1-IX-2018, colecta manual, G. Juárez, Bosque estacionalmente seco de colina; $1 \hat{\jmath}$, Talara, Pariñas, $04^{\circ} 34^{\prime} 52^{\prime \prime} \mathrm{S}-81^{\circ} 16^{\prime} 29^{\prime \prime} \mathrm{O}, 20$ msnm, 4-II-2018, colecta manual, G. Juárez, Bosque estacionalmente seco de llanura.

Comentarios. Especie que amplía su distribución a las provincias de Sullana, Paita, Morropón y Talara y que se limita a ecosistemas secos: desierto y bosques estacionalmente secos. Anteriormente citada para las provincias de Piura y Sechura (Juárez-Noé \& González-Coronado, 2018). Los individuos estudiados fueron colectados en Capparis avicennifolia Kunth y Colicodendron scabridum (Kunth) Hutchinson, 1852 (Capparaceae).

Género Rodolia Mulsant, 1850

60. Rodolia cardinalis (Mulsant, 1850) $\dagger$

Material eXAminado. $1 \hat{\jmath}$, Piura, distrito de Castilla, 0516'66"S-8061'67"O, 29 msnm, 21-I-2019, colecta manual, G. Juárez, Bosque estacionalmente seco de llanura.

Comentarios. Nuevo registro para la región Piura, anteriormente citada para la región Lima (González, 2007).

Familia Mordellidae Latreille, 1802

Subfamilia Mordellinae Latreille, 1802

Género Mordella Linnaeus, 1758

61. Mordella sp†

Material examinado. 1 $\hat{\sigma}$, Piura, distrito de Castilla, 0516'66"S-8061'67"O, 29 msnm, 21-I-2019, colecta manual, G. Juárez, Bosque estacionalmente seco de llanura.

Comentarios. El individuo examinado fue colectado sobre hoja de $P$. oleracea.
Familia Tenebrionidae Latreille, 1802

Subfamilia Pimeliinae Latreille, 1802

Género Parapraocis Kulzer, 1958

62. Parapraocis vagecostata (Fairmaire, 1902)*

Material examinado. 2 ઈิ๊, Talara, El Alto, 04¹6'04"S-81 ${ }^{\circ} 13^{\prime} 09^{\prime \prime O}, 1020$ msnm, 4-II-2015, trampa pitfall, G. Juárez, Bosque estacionalmente seco de montaña, 017 TEN-GJN

Comentarios. Especie citada como Parapraocis sp. 1 en Juárez-Noé \& González-Coronado (2018). Hasta el momento la especie se distribuye en bosques estacionalmente secos.

Género Sechuranus Flores \& Giraldo, 2019

63. Sechuranus barbatus (Kaszab, 1964)

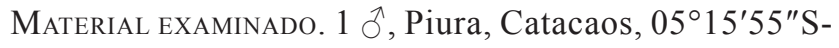
$80^{\circ} 40^{\prime} 30^{\prime \prime} \mathrm{O}, 29 \mathrm{msnm}, 4-\mathrm{II}-2018$, trampa pitfall, G. Juárez, Bosque estacionalmente seco de llanura; 4 ઈิ๊ , Piura, Castilla, caserío Miraflores, 05¹6'66"S-8061'67"O, 29 msnm, 12-IV-2018, trampa pitfall, U. González, Bosque estacionalmente seco de llanura; 1 , Sullana, Ignacio Escudero, 04 $50^{\prime} 35^{\prime \prime} \mathrm{S}-80^{\circ} 52^{\prime} 12^{\prime \prime} \mathrm{O}, 40 \mathrm{msnm}$, 27-VII-2018, trampa pitfall, G. Juárez, Bosque estacionalmente seco de llanura; $2 \hat{\partial} \hat{\sigma}$, Talara, caleta el Ñuro, $04^{\circ} 13^{\prime} 01^{\prime \prime} \mathrm{S}-81^{\circ} 10^{\prime} 35^{\prime \prime} \mathrm{O}, 3 \mathrm{msnm}, 14-\mathrm{VI}-$ 2018, trampa pitfall, G. Juárez, Desierto; $2 \hat{\jmath} \widehat{\jmath}$, Paita, Vichayal, 04 $51^{\prime} 55^{\prime \prime} \mathrm{S}-81^{\circ} 04^{\prime} 19^{\prime \prime} \mathrm{O}, 10 \mathrm{msnm}$, 17-VI-2018, trampa pitfall, G. Juárez, Bosque estacionalmente seco de llanura; $2 \circ \circ$, Sechura, Cristo nos Valga, $05^{\circ} 29^{\prime} 37^{\prime \prime S}-80^{\circ} 44^{\prime} 28^{\prime \prime} \mathrm{O}, 10 \mathrm{msnm}$, 15-VIII-2018, trampa pitfall, G. Juárez, Bosque estacionalmente seco de llanura; 2 우, Morropón, Buenos Aires, $05^{\circ} 66^{\prime} 02^{\prime \prime S}-79^{\circ} 58^{\prime} 03^{\prime \prime} \mathrm{O}, 135 \mathrm{msnm}$, 12-VIII-2018, trampa pitfall, G. Juárez, Bosque estacionalmente seco de colina; 1 + , Ayabaca, Suyo, 0430'45"S-8002'100"O, 450 msnm, 15-XII-2018, trampa pitfall, G. Juárez, Bosque estacionalmente seco de colina; 1 , Huancabamba, Huarmaca, El Overal, 05²7'24"S-7950'02"O, 500 msnm, 15-I2018, trampa pitfall, G. Juárez, Bosque estacionalmente seco de colina.

Comentarios. Con la reciente revisión del género Prohylitus realizada por Giraldo-Mendoza \& Flores (2019) se llegó a la conclusión de que la especie citada como Prohylitus sp en Juárez-Noé \& González-Coronado (2018) se trata $S$. barbatus. Esta especie se limita hasta el momento en bosques estacionalmente secos. 
Género Ammophorus Guerin, 1830

64. Ammophorus peruvianus Guerin, $1830 \dagger$

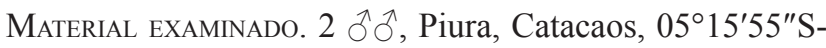
8040'30"O, 29 msnm, 14-II-2019, trampa pitfall, G. Juárez, Bosque estacionalmente seco de llanura;

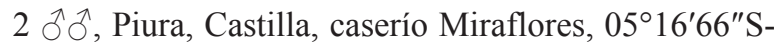
$80^{\circ} 61^{\prime} 67^{\prime \prime} \mathrm{O}, 29 \mathrm{msnm}, 12-\mathrm{I}-2019$, trampa pitfall,

U. González, Bosque estacionalmente seco de llanura; 1 q, Sullana, Ignacio Escudero, 0450'35"S-8052'12"O, 40 msnm, 27-II-2018, trampa pitfall, G. Juárez, Bosque estacionalmente seco de llanura; $1 \hat{\jmath}$, Talara, caleta el Nuro, $04^{\circ} 13^{\prime} 01^{\prime \prime S}-81^{\circ} 10^{\prime} 35^{\prime \prime} \mathrm{O}, 3$ msnm, 23-II-2019, trampa pitfall, G. Juárez, Desierto; 1 đ̃, Paita, Vichayal, 0451'55"S-81 04'19"O, 10 msnm, 27-X-2018, trampa pitfall, G. Juárez, Bosque estacionalmente seco dellanura; 1 o, Sechura, Cristo nos Valga, 05²9'37"S-8044'28"O, 10 msnm, 15-VIII-2018, trampa pitfall, G. Juárez, Bosque estacionalmente seco de llanura; 1 ㅇ, Morropón, Buenos Aires, 0566'02"S-7958'03"O, 135 msnm, 12-IV-2018, trampa pitfall, G. Juárez, Bosque estacionalmente seco de colina; 1 \% , Ayabaca, Suyo, 04³0'45"S-8002'100"O, 450 msnm, 15-XII2018, trampa pitfall, U. González, Bosque estacionalmente seco de colina; 2 우, Huancabamba, Huarmaca, El Overal, $05^{\circ} 27^{\prime} 24^{\prime \prime} \mathrm{S}-79^{\circ} 50^{\prime} 02^{\prime \prime} \mathrm{O}, 500 \mathrm{msnm}, 15-\mathrm{I}-$ 2018, trampa pitfall, G. Juárez, Bosque estacionalmente seco de colina.

Comentarios. Segunda especie del género Ammophorus para la región, anteriormente solo se citaba a Ammophorus rubripes Solier, 1837 (Juárez-Noé \& González-Coronado, 2018). Ambas especies se limitan a bosques estacionalmente secos.

\section{Género Philorea Erichson, 1834}

\section{Philorea mucronata Lesne, 1911}

Material eXaminado. 2 q $q$, Sechura, Cristo nos Valga, 05²9'37"S-8044'28"O, 10 msnm, 15-VI-2018, trampa pitfall, G. Juárez, Desierto costero.

Comentarios. Especie citada como Philorea sp en JuárezNoé \& González-Coronado (2018). La especie se limita solo a desierto costero.

\section{Género Parepitragus Casey, 1907}

66. Parepitragus pulverulentus Erichson, $1847 \dagger$

Material examinado. 1 $\precsim$, Piura, Castilla, caserío Miraflores, $05^{\circ} 16^{\prime} 66^{\prime \prime} \mathrm{S}-80^{\circ} 61^{\prime} 67^{\prime \prime} \mathrm{O}, 29 \mathrm{msnm}$, 2-III-2019, trampa pitfall, U. González, Bosque estacionalmente seco de llanura; $1 \hat{\jmath}$, Sullana, Marcavelica, $04^{\circ} 52^{\prime} 54^{\prime \prime} \mathrm{S}-80^{\circ} 42^{\prime} 12^{\prime \prime} \mathrm{O}, 40 \mathrm{msnm}$,
6-II-2019, trampa pitfall, G. Juárez, Bosque estacionalmente seco de llanura; 1 \% , Sullana, Ignacio Escudero, 0450'35"S-8052'12"O, 40 msnm, 7-I-2019, trampa pitfall, G. Juárez, Bosque estacionalmente seco de lla-

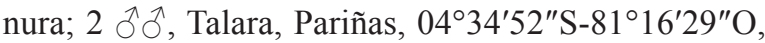
20 msnm, 4-VI-2018, trampa pitfall, G. Juárez, Bosque estacionalmente seco de llanura; $1 \hat{\jmath}$, Paita, Vichayal, 0451'55"S-8104'19"O, 10 msnm, 17-VII-2018, trampa pitfall, G. Juárez, Bosque estacionalmente seco de llanura; 2 우, Sechura, Cristo nos Valga, 05²9'37"S-8044'28"O, 10 msnm, 20-XII-2018, trampa pitfall, G. Juárez, Desierto; 1 q, Morropón, Buenos Aires, $05^{\circ} 66^{\prime} 02^{\prime \prime} \mathrm{S}-79^{\circ} 58^{\prime} 03^{\prime \prime} \mathrm{O}, 135 \mathrm{msnm}$, 12-IV-2018, trampa pitfall, G. Juárez, Bosque estacionalmente seco de colina; 1 , 1 ô, Ayabaca, Suyo, 04³0'45"S-8002'100"O, 450 msnm, 15-XI-2018, trampa pitfall, G. Juárez, Bosque estacionalmente seco de colina; 2 우, Huancabamba, Huarmaca, El Overal, 05²7'24"S-79 50'02"O, 500 msnm, 15-I-2018, trampa pitfall, G. Juárez, Bosque estacionalmente seco de colina.

Comentarios. Segunda especie del género Parepitragus para la región, anteriormente solo se citaba a Parepitragus macrophtalmus Marcuzzi, 1961 (JuárezNoé \& González-Coronado, 2018). Ambas especies se limitan a bosques estacionalmente secos, desierto costero y manglares.

Subfamilia Tenebrioninae Latreille, 1802

Género Tribolium Macleay, 1825

67. Tribolium castaneum (Herbst, 1797) $\dagger$

Material exAminado. 1 ô, Piura, Catacaos, 05¹5'55"S-8040'30"O. 29 msnm, 4-I-2018, colecta manual, G. Juárez, Bosque estacionalmente seco de llanura.

Comentarios. Especie considerada como plaga de productos almacenados (Hagstrum et al., 2013). El individuo estudiado fue colectado en un paquete de harina de pescado.

Género Alphitobius Stephens, 1832

68. Alphitobius diaperinus (Panzer, 1797) $\dagger$

Material examinado. 1 $\precsim$, Piura, Castilla, caserío Miraflores, $05^{\circ} 16^{\prime} 66^{\prime \prime} \mathrm{S}-80^{\circ} 61^{\prime} 67^{\prime \prime} \mathrm{O}, 29 \mathrm{msnm}, 2-\mathrm{V}-2018$, trampa pitfall, U. González, Bosque estacionalmente seco de llanura; 1 $\hat{\jmath}$, Sullana, Marcavelica, 04 52'54"S$80^{\circ} 42^{\prime} 12^{\prime \prime} \mathrm{O}, 40 \mathrm{msnm}, 6-\mathrm{II}-2018$, trampa pitfall, G. Juárez, Bosque estacionalmente seco de llanura; 1 , Sullana, Ignacio Escudero, 0450'35"S-8052'12"O, 
40 msnm, 7-VI-2018, trampa pitfall, G. Juárez, Bosque estacionalmente seco de llanura; $2 \hat{\delta} \hat{\partial}$, Talara, Pariñas,

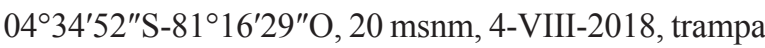
pitfall, G. Juárez, Bosque estacionalmente seco de lla-

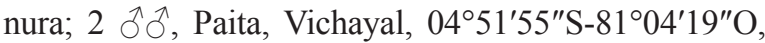
10 msnm, 17-VII-2018, trampa pitfall, G. Juárez, Bosque estacionalmente seco de llanura; 2 q $q$, Sechura,

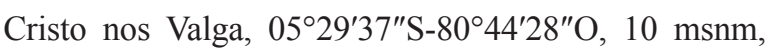
20-XII-2018, trampa pitfall, G. Juárez, Bosque estacionalmente seco de llanura; 2 우, Morropón, Santa Catalina de Mossa, $05^{\circ} 06^{\prime} 10^{\prime \prime} \mathrm{S}-79^{\circ} 53^{\prime} 06^{\prime \prime} \mathrm{O}$, 1000 msnm, 19-VIII-2018, trampa pitfall, G. Juárez, Bosque seco interandino; 1 q, Morropón, Ñoma, 0501'45"S-7952'34"O, 2120 msnm, 19-VIII-2018, trampa pitfall, G. Juárez, Bosque húmedo de montaña; 2 ふึ่, Huancabamba, Canchaque, Pampa Minas, 05²1'20.6"S-79³5'20"O, 2100 msnm, 17-XI-2018, trampa pitfall, G. Juárez, Bosque húmedo de montaña;

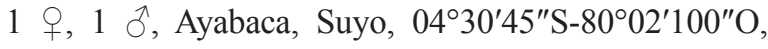
450 msnm, 15-XI-2018, trampa pitfall, G. Juárez, Bosque estacionalmente seco de colina.

Comentarios. Especie de amplia distribución en la región abarcando todas las provincias y paisajes ecológicos. Se le considera plaga importante que afecta a granjas avícolas y granos almacenados (Hagstrum et al., 2013). La mayoría de los individuos fueron colectados en granjas avícolas para la crianza de Gallus gallus Linnaeus, 1758 y Meleagris gallopavo Linnaeus, 1758, sin embargo, algunos individuos también fueron colectados debajo de excremento seco de ganado vacuno.

Género Alaetrinus Iwan, 1995

69. Alaetrinus pullus (Sahlberg, 1823) $†$ (Fig. 4)

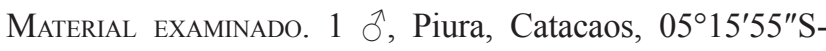
$80^{\circ} 40^{\prime} 30^{\prime \prime} \mathrm{O}, 29 \mathrm{msnm}, 4-\mathrm{I}-2018$, trampa pitfall, G. Juárez, Bosque estacionalmente seco de llanura; $4 \hat{\delta} \hat{\partial}$, Piura, Castilla, caserío Miraflores, $05^{\circ} 16^{\prime} 66^{\prime \prime} \mathrm{S}-80^{\circ} 61^{\prime} 67^{\prime \prime} \mathrm{O}$, 29 msnm, 12-IV-2018, trampa pitfall, U. González, Bosque estacionalmente seco de llanura; $1 \hat{\jmath}$, Piura, Tambogrande, $04^{\circ} 55^{\prime} 38^{\prime \prime} \mathrm{S}-80^{\circ} 21^{\prime} 09^{\prime \prime} \mathrm{O}, 25 \mathrm{msnm}$, 16-III-2018, trampa pitfall, G. Juárez, Bosque estacionalmente seco de llanura; 1 \%, Sullana, Ignacio Escudero,

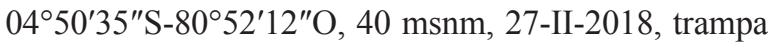
pitfall, G. Juárez, Bosque estacionalmente seco de llanura; 2 ふึَ, Talara, caleta el Nuro, $04^{\circ} 13^{\prime} 01^{\prime \prime} \mathrm{S}-81^{\circ} 10^{\prime} 35^{\prime \prime} \mathrm{O}, 3$ msnm, 14-VI-2018, trampa pitfall, G. Juárez, Desierto;

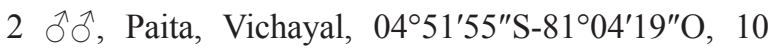
msnm, 17-VII-2018, trampa pitfall, G. Juárez, Bosque estacionalmente seco de llanura; 2 9 , Sechura, Cristo nos Valga, $05^{\circ} 29^{\prime} 37^{\prime \prime} \mathrm{S}-80^{\circ} 44^{\prime} 28^{\prime \prime} \mathrm{O}, 10 \mathrm{msnm}, 25$-VIII2018, trampa pitfall, G. Juárez, Bosque estacionalmente seco de llanura; 2 우, Morropón, Buenos Aires,

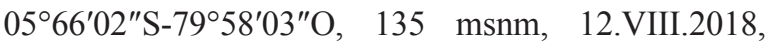
trampa pitfall, G. Juárez, Bosque estacionalmente seco de colina; 1 ㅇ, Ayabaca, Suyo, 04³0'45"S-8002'100"O, 450 msnm, 15-XI-2018, trampa pitfall, G. Juárez, Bosque estacionalmente seco de colina; 1 + Huancabamba, Huarmaca, El Overal, 05²7'24"S-7950'02"O, 500 msnm, 15-I-2018, trampa pitfall, G. Juárez, Bosque estacionalmente seco de colina.

Comentarios. Nuevo registro para Perú, anteriormente citada para México, Guatemala, Belice, Honduras, Cuba, Jamaica, República Dominicana, Puerto Rico, Estados Unidos, Bermudas, Venezuela (Garrido \& Gutiérrez, 1994, 1997; Iwan, 1995, 2002). La especie se distribuye en todas las provincias de la región pero se limita a ecosistemas secos: desierto y bosques estacionalmente secos.

Subfamilia Diaperinae Latreille, 1802

Género Phaleria Latreille, 1804

70. Phaleria psammatea Giraldo-Mendoza, 2019*†

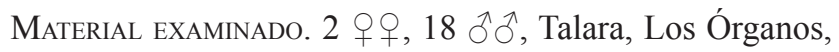
playa los órganos, 4 msnm, 25-X-2018, colecta manual, A. Giraldo, Desierto.

Comentarios. Segunda especie del género Phaleria para la región, anteriormente se citaba a Phaleria pacifica Champion, 1886. Los individuos fueron colectados en cadáveres de Fregata magnificens Mathews, 1914 (Aves: Fregatidae) (Giraldo-Mendoza, 2019).

Referencia. Giraldo-Mendoza (2019).

Familia Oedemeridae Latreille, 1810

Subfamilia Oedemerinae Latreille, 1810

Género Oxycopis Arnett, 1951

71. Oxycopis sp†

Material examinado. $1 \hat{\jmath}$, Piura, Castilla, caserío Miraflores, $05^{\circ} 16^{\prime} 66^{\prime \prime} \mathrm{S}-80^{\circ} 61^{\prime} 67^{\prime \prime} \mathrm{O}, 29 \mathrm{msnm}, 12-\mathrm{IV}-$ 2018, colecta manual, G. Juárez, Bosque estacionalmente seco de llanura.

Familia Meloidae Gyllenhal, 1810

Subfamilia Nemognathinae Laporte, 1840

Género Cissites Latreille, 1804

72. Cissites maculata (Swederus, 1787)

Material examinado. 1 , Sullana, Ignacio Escudero,

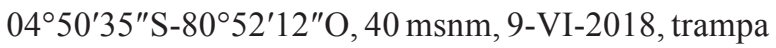
luz, G. Juárez, Bosque estacionalmente seco de llanura; 
2 ふ઼ે, Talara, caleta el Nuro, $04^{\circ} 13^{\prime} 01^{\prime \prime} \mathrm{S}-81^{\circ} 10^{\prime} 35^{\prime \prime} \mathrm{O}$, 3 msnm, 19-VIII-2018, trampa luz, G. Juárez, Desierto;

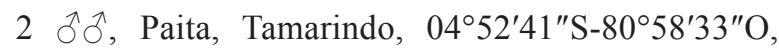
10 msnm, 11-VI-2018, trampa luz, G. Juárez, Bosque estacionalmente seco de llanura; 2 우우, Huancabamba, Canchaque, Cerro Huayanay, 05²4'07"S-79³6'22"O, 1600 msnm, 2-III-2018, trampa luz, G. Juárez, Bosque húmedo de montaña;

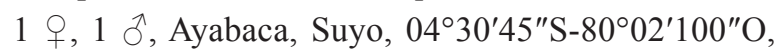
450 msnm, 5-XII-2018, trampa luz, G. Juárez, Bosque estacionalmente seco de colina; 2 우, Morropón, Santa Catalina de Mossa, 0506'10"S-79 53'06"O, 1000 msnm, 19-VIII-2018, trampa luz, G. Juárez, Bosque seco interandino.

Comentarios. Con estos registros la especie abarca ahora todas provincias y paisajes ecológicos de la región. Anteriormente se citaba en las provincias de Piura, Sechura y en los paisajes ecológicos de bosques estacionalmente secos y manglar (Juárez-Noé \& GonzálezCoronado, 2018).

Familia Cerambycidae Latreille, 1802

Subfamilia Prioninae Latreille, 1804

Género Prionacalus White, 1845

73. Prionacalus inermus Komiya \& Santos-Silva, 2018†*

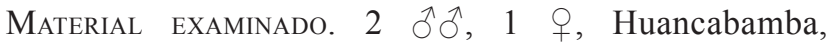
0450'35"S-8052'12"O, 3000 msnm, III-2017, colecta manual, A. Corrales, Bosque húmedo de montaña.

Comentarios. Segunda especie de Prionacalus para la región, anteriormente se citaba a Prionacalus cacicus White, 1845 (Juárez-Noé \& González-Coronado, 2018). Ambas especies se distribuyen en bosques húmedos de montaña.

REFERENCIA. Komiya \& Santos-Silva (2018).

Subfamilia Cerambycinae Latreille, 1802

Género Heterachthes Newman, 1840

74. Heterachthes pallidipennis (Thomson, 1865)

Material examinado. 1 q, Sullana, Ignacio Escudero, 0450'35"S-8052'12"O, 40 msnm, 9-IX-2018, colecta manual, G. Juárez, Bosque estacionalmente seco de lla-

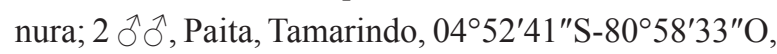
10 msnm, 13-X-2018, colecta manual, G. Juárez, Bosque estacionalmente seco de llanura.

Comentarios. Especie que amplía su distribución a las provincias de Sullana y Paita y se limita a los bosques estacionalmente secos, anteriormente citada para las provincias de Piura y Talara (Juárez-Noé \& GonzálezCoronado, 2018).
Género Smodicum Haldeman, 1847

\section{Smodicum brunneum Thomson, 1878}

Material examinado. 1 ô, Piura, Castilla, Campus Universidad Nacional de Piura, 05¹0'51"S$80^{\circ} 37^{\prime} 08^{\prime \prime} \mathrm{O}, 29 \mathrm{msnm}, 23-\mathrm{III}-2016$, colecta manual, U. González, Bosque estacionalmente seco de llanura, 054 CER-GJN.

Comentarios. Especie citada como Smodicum sp. 1 en Juárez-Noé \& González-Coronado (2018). Hasta el momento la especie se distribuye en bosques estacionalmente secos.

Subfamilia Lamiinae Latreille, 1825

Género Bisaltes Thomson, 1860

76. Bisaltes (Bisaltes) fuscomarmoratus Breuning, 1966

Material eXAminado. 1 + 1 $\widehat{\sigma}$, Ayabaca, Bosque de Cuyas, 043'ㅇ'S-7942'O, $2715 \mathrm{msnm}$, 23-VI-2014, colecta manual, G. Juárez, Bosque húmedo de montaña, 037 CER-GJN.

Comentarios. Especie citada como Bisaltes sp. 1 en JuárezNoé \& González-Coronado (2018). Hasta el momento la especie se distribuye en bosques húmedos de montaña.

Género Taeniotes Audinet-Serville, 1835

77. Taeniotes marmoratus Thomson, 1865 (Fig. 5)

Material examinado. $1 \hat{\jmath}$, Ayabaca, Bosque de Cuyas,

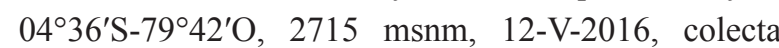
manual, I. Saldaña, Bosque húmedo de montaña, 058 CER-GJN.

Comentarios. Especie citada como Taeniotes sp. 1 en Juárez-Noé \& González-Coronado (2018). Nuevo registro para Perú, anteriormente citada para Ecuador (Monné, 2018). Hasta el momento la especie se distribuye en bosques húmedos de montaña.

Género Desmiphora Audinet-Serville, 1835

78. Desmiphora (Desmiphora) xerophila Martins \& Galileo, $1995 \dagger$

Material examinado. 1 q, Morropón, Buenos Aires, 0566'02"S-79 58'03"O, 135 msnm, 12-XI-2018, colecta manual, G. Juárez, Bosque estacionalmente seco de colina.

Género Estola Fairmaire-Germain, 1859

79. Estola sp $\dagger$

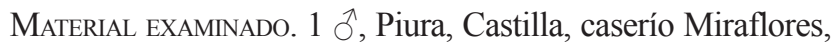
$05^{\circ} 16^{\prime} 66^{\prime \prime} \mathrm{S}-80^{\circ} 61^{\prime} 67^{\prime \prime} \mathrm{O}, \quad 29 \mathrm{msnm}, \quad 12-\mathrm{III}-2019$, colecta manual, G. Juárez, Bosque estacionalmente seco de llanura. 
Género Atrypanius Bates, 1864

80. Atrypanius implexus (Erichson, 1847) $\dagger$

Material examinado. 1 $\sigma^{\lambda}$, Piura, Castilla, $05^{\circ} 11^{\prime} 05^{\prime \prime} \mathrm{S}$ - 8057'27"O, 29 msnm, 25-III-2019, colecta manual, G. Juárez, Bosque estacionalmente seco de llanura.

Familia Chrysomelidae Latreille, 1802

Subfamilia Bruchinae Latreille, 1802

Género Acanthoscelides Schilsky, 1905

81. Acanthoscelides suramerica Johnson, 1990†

Material examinado. 1 त, Piura, Tambogrande, 045'ㅇ's ${ }^{\circ} \mathrm{S}-80^{\circ} 21^{\prime} 09^{\prime \prime} \mathrm{O}, 25$ msnm, 11-II-2018, colecta manual, G. Juárez, Bosque estacionalmente seco de llanura.

Comentarios. Segunda especie de Acanthoscelides para la región, anteriormente se citaba a Acanthoscelides obtectus (Say, 1831) (Juárez-Noé \& GonzálezCoronado, 2018). El individuo estudiado fue colectado en semillas de Leucaena sp. (Fabaceae).

Género Zabrotes Horn, 1885

82. Zabrotes subfasciatus (Boheman, 1833) $\dagger$

Material EXAMINAdo. 1 +, Sechura, Rinconada-Llicuar, 05²7'49"S-8045'55"O, 10 msnm, 13-III-2019, colecta manual, G. Juárez, Bosque estacionalmente seco de llanura.

Subfamilia Alticinae Latreille, 1802

Género Disonycha Chevrolat, 1836

83. Disonycha camposi Barber, $1936 \dagger$

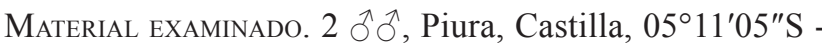
$80^{\circ} 57^{\prime} 27^{\prime \prime}$, 29 msnm, 5-VII-2018, colecta manual, U. González, Bosque estacionalmente seco de llanura; 1 + , Sullana, Marcavelica, 0452'54"S-8042'12"O, 40 msnm, 7-VII-2018, colecta manual, G. Juárez, Bosque estacionalmente seco de llanura; 1 q, Sullana, Bellavista, $04^{\circ} 53^{\prime} 24^{\prime \prime} \mathrm{S}-80^{\circ} 40^{\prime} 51^{\prime \prime} \mathrm{O}, 40 \mathrm{msnm}$, 20-IX-2018, colecta manual, G. Juárez, Bosque estacionalmente seco de llanura; $2 \hat{\jmath}$, Paita, El Arenal, 04 53'03"S-8101'37"O, 10 msnm, 4-IV2018, colecta manual, G. Juárez, Bosque estacionalmente seco de llanura; $2 \hat{\jmath} \widehat{\jmath}$, Paita, Tamarindo, 045' $41^{\prime \prime} \mathrm{S}-80^{\circ} 58^{\prime} 33^{\prime \prime} \mathrm{O}, 10$ msnm, 6-VII-2018, colecta manual, G. Juárez, Bosque estacionalmente seco de llanura; 2 우, Sechura, RinconadaLlicuar, $05^{\circ} 27^{\prime} 49^{\prime \prime} \mathrm{S}-80^{\circ} 45^{\prime} 55^{\prime \prime} \mathrm{O}, \quad 10 \mathrm{msnm}$, 25-VIII-2018, colecta manual, G. Juárez, Bosque estacionalmente seco de llanura; 2 우, Morropón, Buenos Aires, $05^{\circ} 66^{\prime} 02^{\prime \prime} \mathrm{S}-79^{\circ} 58^{\prime} 03^{\prime \prime} \mathrm{O}, 135 \mathrm{msnm}$,
12-VIII-2018, colecta manual, G. Juárez, Bosque estacionalmente seco de colina; $2 \hat{\jmath} \widehat{\jmath}$, Talara, Pariñas,

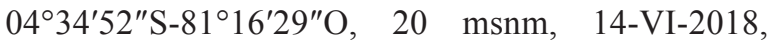
colecta manual, G. Juárez, Bosque estacionalmente seco de llanura.

Comentarios. Los individuos estudiados fueron colectados alimentándose sobre hojas de Alternanthera pungens Kunth, 1817 (Amaranthaceae), Portulaca oleracea L., 1753 (Portulacaceae) y Plantago major L. (Plantaginaceae).

Género Parasyphraea Bechyné, 1959

\section{Parasyphraea sp. $\dagger$}

Material examinado. 1 ô, Morropón, Caracucho, $05^{\circ} 14^{\prime} 02^{\prime \prime S}-79^{\circ} 91^{\prime} 56^{\prime \prime} \mathrm{O}, 650 \mathrm{msnm}, 17-\mathrm{VIII}-2018$, colecta manual, U. González, Bosque estacionalmente seco colina.

Subfamilia Eumolpinae Hope, 1840

Género Myochrous Dejean, 1837

85. Myochrous geminus Blake, 1950

Material examinado. $2 \hat{\jmath} \hat{0}$, Piura, Castilla, Campus Universidad Nacional de Piura, 05²10'51"S$80^{\circ} 37^{\prime} 08^{\prime \prime}$ O. 29 msnm, 17-VI-2016, colecta manual, U. González, Bosque estacionalmente seco de llanura, 029 CHR-GJN.

Comentarios. Especie citada como Myochrous sp. 1 en Juárez-Noé \& González-Coronado (2018). Hasta el momento la especie se distribuye en bosques estacionalmente secos.

Subfamilia Galerucinae Latreille, 1802

Género Cochabamba Bechyné, 1955

86. Cochabamba chrysopleura (Harold, 1875)†

Material examinado. 1 ej., Huancabamba, 05²4'07"S-79³6'22"O, 1600 msnm, Bosque húmedo de montaña.

REFERENCIA. Rocha (2010).

Subfamilia Cryptocephalinae

Género Coscinoptera Lacordaire, 1848

\section{Coscinoptera sp. $\dagger$}

Material exAminado. 1 ô, Piura, Castilla, 05 $11^{\circ} 05^{\prime \prime} \mathrm{S}-80^{\circ} 57^{\prime} 27^{\prime \prime} \mathrm{O}, 29$ msnm, 5-VII-2018, colecta manual, G. Juárez, Bosque estacionalmente seco de llanura.

Comentarios. Nuevo registro para Perú. El género se distribuye en México, Costa Rica, Panamá, Colombia, Venezuela, Brasil, Argentina, Paraguay y Uruguay (Moldenke, 1981). 
Familia Anthribidae Billberg, 1820

Subfamilia Choraginae Kirby, 1819

Género Araecerus Schöenherr, 1823

88. Araecerus fasciculatus (DeGeer, 1775) $\dagger$

Material examinado. $2 \hat{\jmath} \hat{\jmath}$, Huancabamba, Canchaque, Pampa Minas, $05^{\circ} 21^{\prime} 20.6^{\prime \prime} \mathrm{S}-79^{\circ} 35^{\prime} 20^{\prime \prime} \mathrm{O}, \quad 2100$ msnm, 7-XI-2018, colecta manual, G. Juárez, Bosque húmedo de montaña; 1 +, 1 , Ayabaca, Suyo, 0430'45"S-8002'10"O, 450 msnm, 25-IX-2018, colecta manual, G. Juárez, Bosque estacionalmente seco de colina.

Comentarios. Primera especie de Anthribidae para la región. Especie considerada plaga importante en el cultivo de café (Hagstrum et al., 2013). Los individuos estudiados fueron colectados en granos almacenados de café.

Familia Dryophthoridae Schönherr, 1833

Subfamilia Rhynchophorinae Schönherr, 1833

Género Rhynchophorus Herbst, 1795

89. Rhynchophorus palmarum (Linnaeus, 1758)

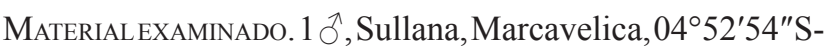
$80^{\circ} 42^{\prime} 12^{\prime \prime} \mathrm{O}, 40 \mathrm{msnm}, 22-\mathrm{X}-2018$, trampa pitfall, G. Juárez, Bosque estacionalmente seco de llanura.

Comentarios. Especie que amplía su distribución a la provincia de Sullana y se limita a los bosques estacionalmente secos. Anteriormente citada para la provincia de Piura (Juárez-Noé \& González Coronado, 2018). El individuo estudiado se colectó en Cocos nucifera L., 1793 (Arecaceae).

Familia Curculionidae Latreille, 1802

Subfamilia Entiminae Schöenherr, 1823

Género Eustylus Sahlberg, 1823

90. Eustylus humilis (Erichson, 1847)

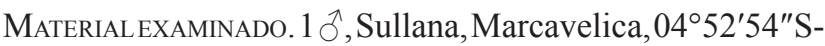
$80^{\circ} 42^{\prime} 12^{\prime \prime} \mathrm{O}, 40 \mathrm{msnm}, 11-\mathrm{XII}-2018$, trampa pitfall, G. Juárez, Bosque estacionalmente seco de llanura;

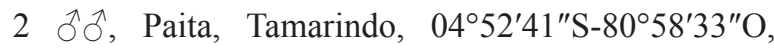
10 msnm, 16-X-2018, trampa pitfall, G. Juárez, Bosque estacionalmente seco de llanura.

COMENTARIOS. Especie que amplía su distribución a las provincias de Sullana y Paita y se limita a los bosques estacionalmente secos. Anteriormente citada para la provincia de Piura (Juárez-Noé \& González Coronado, 2018).

Género Brachyomus Lacordaire, 1863

\section{Brachyomus sp $\dagger$}

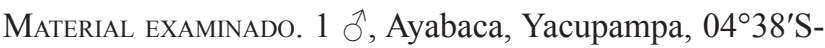
7943'O, 2715 msnm, 11-VIII-2018, colecta manual, G. Juárez, Bosque húmedo de montaña.
Género Naupactus Dejean, 1821

\section{Naupactus sp $\dagger$}

Material examinado. 1 ô, Piura, $05^{\circ} 12^{\prime} \mathrm{S}-80^{\circ} 37^{\prime} \mathrm{O}, 39$ msnm, 10-II-2018, colecta manual, G. Juárez, Bosque estacionalmente seco de llanura.

Subfamilia Baridinae Schönherr, 1836

Género Eurhinus Illiger, 1807

93. Eurhinus festivus (Fabricius, 1792) $\dagger$ (Fig. 6)

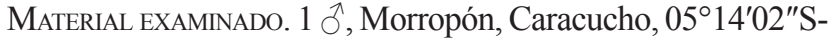
7991'56"O, 650 msnm, 17-VIII-2018, colecta manual, U. González, Bosque estacionalmente seco colina.

Comentarios. Segunda especie para la subfamilia Baridinae, anteriormente se citaba a Linogeraeus perscitus (Herbst, 1797) (Juárez-Noé \& González-Coronado, 2018).

Subfamilia Scolytinae Latreille, 1804

Género Pagiocerus Eichhoff, 1868

94. Pagiocerus frontalis (Fabricius, 1801) $\dagger$

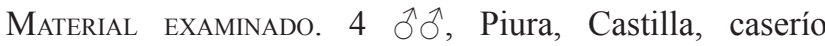
Miraflores, $05^{\circ} 16^{\prime} 66^{\prime \prime} \mathrm{S}-80^{\circ} 61^{\prime} 67^{\prime \prime} \mathrm{O}, 29 \mathrm{msnm}, 10$-IV2018, colecta manual, G. Juárez, Bosque estacionalmente seco de llanura.

Comentarios. Nuevo registro para la región Piura, anteriormente citada para la región Huánuco (Smith et al., 2017). Especie considerada plaga de productos almacenados (Hagstrum et al., 2013). Los individuos estudiados fueron colectados en granos almacenados de Zea mays L. (Poaceae).

Género Hypothenemus Westwood, 1834

95. Hypothenemus hampei (Ferrari, 1867) $\dagger$

Material examinado. 1 $\hat{\jmath}$, Huancabamba, Canchaque, Pampa Minas, 05²1'20.6"S-79³5'20"O, 2100 msnm, 7-XI-2018, colecta manual, G. Juárez, Bosque húmedo de montaña.

Comentarios. Esta especie es considerada plaga del cultivo de café atacando principalmente el fruto (BustilloPardey, 2006).

En cuanto a los métodos de captura, 41 especies (79\% de las colectadas) se recogieron a través de colecta manual, siendo 37 de ellas exclusivas de este método, mientras que el $21 \%$ restante se recogieron a través de trampas pitfall ( 7 especies, 6 de ellas exclusivas), trampas de luz (7 especies, 4 de ellas exclusivas) y con red acuática (2 especie, 1 exclusiva).

La colecta manual es un potencial para el monitoreo de este grupo de insectos, ya que el colector busca de manera activa a los organismos en su ambiente abarcando así la 
mayor cantidad de hábitat disponibles. Con este método se capturó exclusivamente a las especies que dañan productos almacenados tales como $T$. castaneum, $A$. advena, $P$. frontalis, D. maculatus, $S$. paniceum, los que se alimentan de cadáveres en descomposición como $D$. ater y D. frischii y las especies que frecuentan el follaje como $S$. anale, $H$. pallidipennis que barrenan tallos y troncos, los coccinéllidos A. orbigera ecuadorica, S. ecuadoricus, $P$. insidiosa, B. darlene, C. maculata bisexpunctata, H. axyridis que se alimentan de otros insectos actuando como controladores biológicos y C. repandus, A. suramerica, A. fasciculatus, G. merops, D. camposi, H. hampei, $M$. actaeon, D. neptunus, Naupactus sp, Brachyomus sp, Coscinoptera sp, Z. subfasciatus y $S$. aloeus que se alimentan de tallos, hojas, flores y frutos. Sin embargo, las especies que se alimentan de hojas, flores, frutos y de otros insectos también pueden ser capturadas a través de mangueo con red entomológica, método que no se utilizó en este trabajo.

Del mismo modo, R. palmarum, E. humilis A. pullus, y A.diaperinus, S. barbatus, A.peruvianus, P. pulverulentus se capturaron con trampas pitfall, método ideal para la captura de insectos que frecuentan la superficie y hojarasca, mientras que con las trampas de luz se colectó a $M$. arrowi, P. ornaticornis, C. maculata, $C$. chrysochlora y A. pseudolividus, especies generalmente con mayor actividad nocturna, aunque $A$. pseudolividus también se capturó a través de colecta manual en heces frescas de ganado vacuno. Con red acuática se capturó a A. peruvianus y $M$. calidus, aunque esta última también se colectó con trampas luz, mientras que G. aegeon fue colectada simultáneamente a través de colecta manual, trampas pitfall y trampas de luz.

\section{Agradecimientos}

A Caroline Chaboo por el envío de listas de especies por familia del Proyecto Beetles (Coleoptera) of Peru. A Gianni Allegro (Carabidae), Rob Westerduijn, Wills Flowers (Chrysomelidae), Paul Johnson (Elateridae), Charles Hart (Tenebrionidae), Carlos A. Julio, Jhon Cesar Neita, Brett Ratcliffe (Scarabaeidae) y Robert Constantin (Melyridae y Cantharidae) por el envío de material bibliográfico y ayuda en la identificación de algunos especies. Al biólogo Irwing S. Saldaña por su fotografía de T. marmoratus.

\section{Referencias}

Ahn, K-J. \& Ashe, J. S., 1996. A revision of Rothium Moore and Legner (Coleoptera: Staphylinidae: Aleocharinae) with a discussion of its phylogenetic relationships. Journal of the Kansas Entomological Society, 69(3): 234-256. http://www.jstor.org/ stable/25085676
Arnaud, P. P. \& Bomans, H., 2006. Descriptions de trois nouvelles espèces de coléoptères Lucanidae du Pérou. Besoiro, 15: 2-5.

Arriagada, G., 2015. Nueva especie de Saprinae para Perú, Chile y Argentina, nuevos registros y sinonimias (Coleoptera: Histeridae). Boletín del Museo Nacional de Historia Natural, Chile, 64: 185-201.

Asiain, J. Márquez, J. \& Morrone, J. J., 2010. Track analysis of the species of Agrodes and Plochionocerus (Coleoptera: Staphylinidae). Revista Mexicana de Biodiversidad, 81(1): 177- 181

Barber, H. S., 1936. A new ecuadorian fleabeetle injuring crucifers (Coleoptera: Chrysomelidae). Journal of the Washington Academy of Sciences, 26(4): 181-182.

Blake, D. H., 1950. A revision of the beetles of the genus Myochrous. Proceedings of the United States National Museum, 101(3271): 1-64. https://doi.org/10.5479/ si.00963801.101-3271.1

Benetti, C., Régil. J. \& Garrido, J., 2003. Estudio faunístico de Hydradephaga (Coleoptera: Dytiscidae, Gyrinidae, Haliplidae, Noteridae) en el municipio de Gramado, Sur de Brasil. Boletín de la Sociedad Entomológica Aragonesa (S.E.A.), 32: 37-44.

Bouchard, P., Bousquet, Y., Davies, A., Alonso-Zarazaga, M.A., Lawrence, J., Lyal, C., Newton, A., Reid, C., Schmitt, M., Slipinski, A. \& Smith, A., 2011. Family-group names in Coleoptera (Insecta). ZooKeys, 88: 1-972. https://doi.org/10.3897/zookeys.88.807

Boucomont A., 1932. Synopsis des Onthophagus d'Amérique du Sud (Coleoptera, Scarabaeidae). Annales de la Société Entomologique de France, 101(4):293-332.

Breuning, S., 1966. Nouvelles formes de Lamiaires (Dix-Septieme Partie). Bulletin Institut Royal des Sciences Naturelles de Belgique, 42(21): 1-24.

Brinck, P., 1977. Evolution and taxonomy of Andogyrus Ochs (Coleoptera: Gyrinidae). Entomologica Scandinavica, 8: 241-269. https://doi. org/10.1163/187631277X00369

Bustamante-Navarrete, A., Oros-Ramos, A., Yábar-Landa, E., Marquina-Montesinos, E. L. \& Elme-Tumpay, A., 2017. Contribución al conocimiento de los escarabajos de la familia Silphidae (Coleoptera) en el Perú. Arquivos Entomolóxicos, 17: 135-143.

Bustamante, A., 2018. Registro de los coleópteros acuáticos en la región Cusco, Perú. The Biologist (Lima), 16(1): 35-49. https://doi.org/10.24039/rtb2018161220

Bustillo Pardey, A. E., 2006. Una revisión sobre la broca del café, Hypothenemus hampei (Coleoptera: Curculionidae: Scolytinae), en Colombia. Revista Colombiana de Entomología, 32(2): 101-116.

Casari, S., 2002. Review of the genus Chalcolepideus Eschscholtz, 1829 (Coleoptera, Elateridae, Agrypninae). Revista Brasileira de Entomologia, 46(3): 263-428. https://doi.org/10.1590/S0085-56262002000300007

Chaboo, C., 2015. Beetles (Coleoptera) of Peru: A Survey of the Families. Part I. Overview. Journal of the Kansas Entomological Society, 88(2): 135-139. https://doi. org/10.2317/0022-8567-88.2.135 
Chandler, D. 2016. Beetles (Coleoptera) of Peru: A Survey of the Families. Anthicidae Latreille, 1824. Journal of the Kansas Entomological Society, 89(2): 182-190. https://doi.org/10.2317/150920.1

Chani-Posse, M. \& Asenjo, A. 2013. Systematics and phylogeny of the Andean genera Leptopeltus Bernhauer and Leptopeltoides gen. nov. (Coleoptera: Staphylinidae), with biogeographical notes. Zoologischer Anzeiger, 252: 440-456. https://doi.org/10.1016/j.jcz.2013.02.001

Ciro-Diaz, W. Anteparra, M.E. \& Hermann, A., 2008. Dermestidae (Coleoptera) en el Perú: revisión y nuevos registros. Revista Peruana de Biología, 15(1): 15-20. https://doi.org/10.15381/rpb.v15i1.1662

Coelho-Grossi, P., 2011. Revisão Taxonômica e Filogenia de Sclerostomini Benesh, 1955 (Coleoptera, Lucanidae, Lucaninae). Tesis doutor em Ciências Biológicas, Universidade Federal do Paraná. Disponible en: https:// acervodigital.ufpr.br/handle/1884/48431

Constantin, R., 2009. A contribution to the genus Silis Charpentier, 1825, in Ecuador (Coleoptera, Cantharidae). Entomologica Basiliensia et Collectionis Frey, 31: 55-87.

Constantin, R., 2011. A contribution to the genus Astylus Laporte de Castelnau, 1836 in Ecuador, with descriptions of three new species (Coleoptera, Melyridae). Entomologica Basiliensia, 33: 39-61.

Dillon, L. S. \& Dillon, E. S., 1941. The tribe Monochamini in the Western Hemisphere (Coleoptera: Cerambycidae). Scientific Publications of the Reading Public Museum, 1: 1-135. Disponible en: https://babel.hathitrust.org/cgi/pt?id=wu.89095282059; view=1up;seq=9

Furth, D., Savini, V. \& Chaboo, C., 2015. Beetles (Coleoptera) of Peru: A Survey of the Families. Chrysomelidae: Alticinae (Flea Beetles). Journal of the Kansas Entomological Society, 88(3): 368-374. https://doi.org/10.2317/ kent-88-03-368-374.1

Garrido, O. H. \& Gutiérrez, E., 1994. A new species of Opatrinus (Coleoptera: Pedinini: Tenebrionidae) from Cuba. Insecta Mundi, 8(1-2): 121-124. Disponible en: http:// journals.fcla.edu/mundi/article/view/24752/24083

Garrido, O. H. \& Gutiérrez, E., 1997. Consideraciones sobre el género Opatrinus (Coleoptera: Tenebrionidae: Pedinini) en Cuba, Puerto Rico y Jamaica. Insecta Mundi, 11(1): 25-27. Disponible en: http://digitalcommons.unl.edu/cgi/viewcontent. cgi article $=1253 \&$ context $=$ insectamundi

Gidaspow, T., 1963. The genus Calosoma in Central America, The Antilles and South America (Coleoptera, Carabidae). Bulletin of the American Museum of Natural History, 124(7): 1-44. Disponible en: http://digitallibrary.amnh.org/handle/2246/1214

Giraldo, A. \& Arellano, G., 2003. Resiliencia de la comunidad epígea de Coleoptera en las Lomas de Lachay después del evento El Niño 1997-98. Ecología Aplicada, 2(1): 59-68. Disponible en: http://www.lamolina.edu. pe/ecolapl/Resilencia_coleopteros_Lachay.htm

Giraldo-Mendoza, A., 2019. New species of the genus Phaleria Latreille (Tenebrionidae: Diaperinae) from northern coast of Peru. Revista Chilena de Entomología, 45(2): 277-282.
Giraldo-Mendoza, A. E. \& Flores, G., 2019. A revision of the Peruvian Edrotini (Coleoptera: Tenebrionidae: Pimeliinae). Annales Zoologici (Warszawa), 69(1): 83-98.

Giraldo, A. \& Flores, E., 2016. Peruvian Tenebrionidae: a review of present knowledge and Biodiversity. Annales Zoologici (Warszawa), 66(4): 499-513. https://doi.org/1 0.3161/00034541ANZ2016.66.4.002

González, G., 2015. Beetles (Coleoptera) of Peru: A Survey of the Families. Coccinellidae. Journal of the Kansas Entomological Society, 88(2): 229-236. https://doi. org/10.2317/kent-88-02-229-236.1

González, G., 2007. Los Coccinellidae de Perú. Disponible en: https:/www.coccinellidae.cl/paginasWebPeru/Paginas/InicioPeru.php

Gordon, R. D. \& González, G., 2005. South American Coccinellidae (Coleoptera). Part IX: A systematic revision of Scymnobius Casey (Scymninae: Scymnini). Frustula Entomologica, 25(35): 57-85.

Gorham, J. R., 1991. Insect and Mite Pest in Food: An Illustrated key. Volume 1. United States Department of Agriculture. Agriculture Handbook No. 655. 319 pp.

Hagstrum, D. W., Klejdysz, T., Subramanyan, B. \& Nawrot, J., 2013. Atlas of Stored-Product Insects and Mites. AACC International, United States of America. 589 pp.

Howden, H. F., 1985. A revision of the south american species in the genus Neoathyreus Howden and Martínez (Coleoptera, Scarabaeidae, Geotrupinae). Contributions of the American Entomological Institute, 21(4): 1-95.

Iwan, D., 1995. Revision of the genus Opatrinus Dejean, 1821 (Coleoptera: Tenebrionidae: Platynotini). Genus, 6(1): $1-90$.

Iwan, D., 2002. Catalogue of the World Platynotini (Coleoptera: Tenebrionidae). Genus, 13(2): 219-323.

Jameson, M. L., 1997. Phylogenetic analysis of the subtribe Rutelina and revision of the Rutela generic groups (Coleoptera: Scarabaeidae: Rutelinae: Rutelini). Bulletin of the University of Nebraska State Museum, 14: 1-186.

Juárez-Noé, G. \& González-Coronado, U., 2018. Lista de coleópteros (Insecta: Coleoptera) de la región Piura, Perú. Folia Entomológica Mexicana (n.s.), 4(1): 1-27.

Kingsolver, J. M., Barriga-Tuñón, J. E., Romero-Nápoles, J. \& Thomas, M., 2017. Bruchidae of Chile (Insecta: Coleoptera). Insecta Mundi, 0542: 1-106.

Komiya, Z. \& Santos-Silva, A., 2018. A new species of Prionacalus White, 1845 from Peru (Coleoptera, Cerambycidae, Prioninae, Prionini). Les Cahiers Magellanes, 31: 18-24.

Kulzer, H., 1958. Monographie der südamerikanische Tribus Praocini (Col.). Entomologische Arbeiten aus dem Museum G. Frey, 9(1): 1-105.

Lachaume, G., 1985. Les coléoptères du Monde, Vol 5, Dynastini. 1. Dynastes-Megasoma-Golofa. Sciences Nat, Venette. 85 pp.

López-García, M., Giraldo-Mendoza, A. \& Deloya, C., 2019. Taxonomic synopsis of the genus Tomarus Erichson, 1847 (Coleoptera: Scarabaeidae: Dynastinae) 
in Perú. Zootaxa, 4604(3): 428-440. https://doi. org/10.11646/zootaxa.4604.3.2

Lozada, A., Fernández, I. \& Trujillo, M., 2004. Lista preliminar de los coleópteros (Insecta: Coleoptera) de Topes de Collantes, Trinidad, Sancti Spíritus, Cuba. Boletín de la Sociedad Entomológica Aragonesa (S.E.A.), 34: 101-106.

Martins, U. R., 1975. A taxonomic revision of the world Smodicini (Coleoptera, Cerambycidae). Arquivos de Zoologia, 26(4): 319-359.

Miller, K. \& Bergsten, L., 2016. Diving Beetles of the World. Johns Hopkins University Press. Baltimore. 320 pp.

Moldenke, A. R., 1981. A generic reclassification of the new world Clytrinae (Coleoptera: Chrysomelidae). Entomologische Arbeiten aus dem Museum G. Frey, 29: 75-116.

Monné, M., 2018. Catalogue of the Cerambycidae (Coleoptera) of the Neotropical Region. Part II. Subfamily Lamiinae. Disponible en: http://cerambyxcat.com/ Parte2_Lamiinae_2018.pdf

Moore, R. M., Cave, R. D. \& Branham, M. A., 2018. Annotated catalog and bibliography of the cyclocephaline scarab beetles (Coleoptera, Scarabaeidae, Dynastinae, Cyclocephalini). ZooKeys, 745: 101-378. https://doi. org/10.3897/zookeys.745.23685

More, A., Villegas, P. \& Alzamora, M., 2014. Piura, Áreas prioritarias para la conservación de la biodiversidad. Naturaleza y Cultura Internacional-PROFONANPE. Lima. 166 pp.

Moore, T. \& Diéguez, V. M., 2019. Adición al conocimiento de los Stigmoderini de Perú (Coleoptera: Buprestidae). Revista Chilena de Entomología, 45(1): 165-173.

Morón, M. A., 2004. Escarabajos, 200 millones de años de evolución. Instituto de Ecología, A.C. y Sociedad Entomológica Aragonesa. Zaragoza. 204 pp.

Nilsson, A. N. \& Hájek, J., 2018. A World Catalogue of the Family Dytiscidae, or the Diving Beetles (Coleoptera, Adephaga). Version 1. I. 2018. Disponible en: http:// www.waterbeetles.eu/documents/W CAT Dytiscidae_2018.pdf

Noonan, G. R., 1981. South American species of the subgenus Anisotarsus Chaudoir (genus Notiobia Perty: Carabidae: Coleoptera). Part I: Taxonomy and Natural History. Contributions in Biology and Geology, 44: $1-84$.

Opitz, W., 2018. Classification, Natural History, and Evolution of the Subfamily Peloniinae OPITZ (Coleoptera: Cleroidea: Cleridae). Part X. Taxonomic revision of the New World genus Pelonium Spinola. Entomofauna, 39(2): 337-527.

Pinto, J. \& Bologna, M., 2016. Beetles (Coleoptera) of Peru. Survey of the families. Meloidae Gyllenhal, 1810. Journal of the Kansas Entomological Society, 89(2): 202-209. https://doi.org/10.2317/JKES151211.1

Puthz, V., 2017. Übersicht über die neotropischen Arten der Gattung Stenus LATREILLE mit seitlich ungerandetem Abdomen und gelappten Tarsen (Coleoptera, Staphylinidae) 351. Beitrag zur Kenntnis der Steninen. Linzer Biologische Beiträge, 49(1): 749-883.
Ratcliffe, B. C., 1976. A revision of the genus Strategus (Coleoptera: Scarabaeidae). Bulletin of the University of Nebraska State Museum, 10(3): 93-204.

Ratcliffe, B. C., 2015. A review of the neotropical genus Marmarina Kirby, 1827 (Coleoptera: Scarabaeidae: Cetoniinae: Gymnetini) with description of a new species from Argentina. The Coleopterist Bulletin, 69(2): 183-201. https://doi. org/10.1649/0010-065X-69.2.183

Ratcliffe, B. C., 2018. A Monographic Revision of the Genus Gymnetis MacLeay, 1819 (Coleoptera: Scarabaeidae: Cetoniinae). Bulletin of the University of Nebraska State Museum, 31: 1-250.

Ratcliffe, B., Jameson, M., Figueroa, L., Cave, R., Paulsen, M., Cano, E., Beza-Beza, C., Jiménez-Ferbans, L. \& Reyes-Castillo, P., 2015. Beetles (Coleoptera) of Peru: A Survey of the Families. Scarabaeoidea. Journal of the Kansas Entomological Society, 88(2): 186-207. https:// doi.org/10.2317/kent-88-02-186-207.1

Rees, D. P., 2004. Insect of Stored Products. Commonwealth Scientific and Industrial Research Organisation. $181 \mathrm{pp}$.

Rocha, L., 2010. Taxonomia e análise cladística de Cochabamba Bechyné, 1955 (Coleoptera, Chrysomelidae, Galerucinae). Tesis maestrado em Ciências, Instituto de Biociências, Universidade de São Paulo. Disponible en: http://www.teses.usp.br/teses/disponiveis/41/41133/ tde-15122010-143004/pt-br.php

Rossini, M., Vaz de Mello, F. Z. \& Zunino, M., 2018. A taxonomic revision of the New World Onthophagus Latreille, 1802 (Coleoptera: Scarabaeidae: Scarabaeinae) of the osculatii species-complex, with description of two new species from South America. Journal of Natural History, 52(9-10): 541-586. https://doi.org/10. 1080/00222933.2018.1437230

Salazar, K. \& Boucher, S., 2018. The exceptionally diversified Neotropical genus Veturius Kaup: more new data on its phylogeny, taxonomy and distribution (Coleoptera: Passalidae). Annales de la Société Entomologique de France (N.S.), 54(5): 1-24. https://doi.org/10.1080/0 0379271.2018 .1514654

Smith, A., 2003. A monographic revision of the genus Platycoelia (Coleoptera: Scarabaeidae: Rutelinae: Anoplognathini). Bulletin of the University of Nebraska State Museum, 15: 1-202.

Smith, A., Mendoza, A., Flores, G. \& Aalbu, R., 2015. Beetles (Coleoptera) of Peru: A Survey of the Families. Tenebrionidae. Journal of the Kansas Entomological Society, 88(2): 221-228. https://doi.org/10.2317/ kent-88-02-221-228.1

Smith, S. M., Petrov, A. V. \& Cognato, A. I., 2017. Beetles (Coleoptera) of Peru: A Survey of the Families. Curculionidae: Scolytinae. The Coleopterist Bulletin, 71(1): 77-94. https://doi.org/10.1649/0010-065X-71.1.77

Solís, A., 2004. Métodos y técnicas de recolecta para coleópteros Scarabaeoideos. Available at: http:// unsm-ento.unl.edu/Escarabajos.htm

Soula, M., 2002. Rutelini 2. Revision of the Anticheirina 2. Les Coléoptères du Monde, 26(2): 117-296. 
Soula, M., 2008. Les Coléoptères du Nouveau Monde. Volume 2: Rutelini 2. Révision des Pelidnotina 2. Révision des genres: Parhoplognathus, Chipita n. gen., Heteropelidnota, Homothermon, Hoplopelidnota, Mesomerodon, Mecopelidnota, Patatra n. gen. (Coleoptera: Scarabaeidae, Rutelinae, Rutelini). Besoiro: Supplément au Bulletin de liaison de l'Association Entomologique pour la Connaissance de la Faune Tropicale. AECFT, Saintry, $40 \mathrm{pp}$.

Thomas, M. C. \& Chaboo, C. S., 2015. Beetles (Coleoptera) of Peru: A Survey of the Families. Cucujidae, Laemophloeidae, Silvanidae, Passandridae (Cucujoidea). Journal of the Kansas Entomological Society, 88(2): 251-257.
Valle da Silva, P. R. \& Massutti de Almeida, L., 2001. Chaves para a Identificação dos principais Coleoptera (Insecta) associados com productos armazenados. Revista Brasileira Zoologia, 18(1): 271-283. http://dx. doi.org/10.1590/S0101-81752001000100031

Vaurie, P., 1982. Revision of neotropical Eurhin (Coleoptera, Curculionidae, Baridinae). American Museum Novitates, 2753: 1-44.

Villarreal, H., Álvarez, M., Córdoba, F., Fagua, G., Gast, F., Mendoza, H., Ospina, M. \& Umaña, A., 2004. Manual de métodos para el desarrollo de inventarios de biodiversidad. Programa de Inventarios de Biodiversidad. Instituto de Investigación de Recursos Biológicos Alexander Von Humboldt. Bogotá. 238 pp. 
Apéndice 1.- Localidades de muestreo de colectas de coleópteros en la región Piura, Perú.

Appendix 1.- Sampling localities of beetles collections in the Piura region, Peru.

\begin{tabular}{|c|c|c|c|c|}
\hline Provincia & Localidad de muestreo & Coordenadas & Paisaje ecológico & Altitud (msnm) \\
\hline \multirow[t]{4}{*}{ Piura } & Distrito Castilla & $05^{\circ} 11^{\prime} 05^{\prime \prime S}-80^{\circ} 57^{\prime} 27^{\prime \prime O}$ & BESII & 30 \\
\hline & Distrito Catacaos & $05^{\circ} 15^{\prime} 55^{\prime \prime S}-80^{\circ} 40^{\prime} 30^{\prime \prime O}$ & BESII & 30 \\
\hline & Distrito Tambogrande & $04^{\circ} 55^{\prime} 38^{\prime \prime S}-80^{\circ} 21^{\prime} 09^{\prime \prime O}$ & BESII & 30 \\
\hline & Caserío Miraflores & $05^{\circ} 16^{\prime} 66^{\prime \prime} \mathrm{S}-80^{\circ} 61^{\prime} 67^{\prime \prime O}$ & BESII & 30 \\
\hline \multirow[t]{4}{*}{ Sullana } & Distrito Marcavelica & $04^{\circ} 52^{\prime} 54^{\prime \prime S}-80^{\circ} 42^{\prime} 12^{\prime \prime O}$ & BESII & 40 \\
\hline & Distrito Ignacio Escudero & $04^{\circ} 50^{\prime} 35^{\prime \prime S}-80^{\circ} 52^{\prime} 12^{\prime \prime O}$ & BESII & 40 \\
\hline & Distrito Miguel Checa & $04^{\circ} 54^{\prime} 08^{\prime \prime S}-80^{\circ} 48^{\prime} 56^{\prime \prime O}$ & BESII & 40 \\
\hline & Distrito Bellavista & $04^{\circ} 53^{\prime} 24^{\prime \prime S}-80^{\circ} 40^{\prime} 51^{\prime \prime O}$ & BESII & 40 \\
\hline \multirow[t]{3}{*}{ Paita } & Distrito El Arenal & $04^{\circ} 53^{\prime} 03^{\prime \prime S}-81^{\circ} 01^{\prime} 37^{\prime \prime O}$ & BESII & $0-20$ \\
\hline & Distrito Tamarindo & $04^{\circ} 52^{\prime} 41^{\prime \prime S}-80^{\circ} 58^{\prime} 33^{\prime \prime O}$ & BESII & $0-20$ \\
\hline & Distrito Vichayal & $04^{\circ} 51^{\prime} 55^{\prime \prime S}-81^{\circ} 04^{\prime} 19^{\prime \prime O}$ & BESIIMAN & $0-20$ \\
\hline \multirow[t]{2}{*}{ Talara } & Distrito Pariñas & $04^{\circ} 34^{\prime} 52^{\prime \prime S}-81^{\circ} 16^{\prime} 29^{\prime \prime O}$ & BESII/DES & $0-100$ \\
\hline & Caleta El Ñuro & $04^{\circ} 13^{\prime} 01^{\prime \prime S}-81^{\circ} 10^{\prime} 35^{\prime \prime O}$ & DES & $0-10$ \\
\hline \multirow[t]{2}{*}{ Sechura } & Distrito Cristo nos Valga & $05^{\circ} 29^{\prime} 37^{\prime \prime} \mathrm{S}-80^{\circ} 44^{\prime} 28^{\prime \prime} \mathrm{O}$ & BESII & 20 \\
\hline & Distrito Rinconada Llicuar & $05^{\circ} 27^{\prime} 49^{\prime \prime S}-80^{\circ} 45^{\prime} 55^{\prime \prime}$ & BESII & 20 \\
\hline \multirow[t]{3}{*}{ Morropón } & Distrito Buenos Aires & $05^{\circ} 66^{\prime} 02^{\prime \prime S}-79^{\circ} 58^{\prime} 03^{\prime \prime O}$ & $\mathrm{BESc} / \mathrm{BESm}$ & $500-1100$ \\
\hline & $\begin{array}{l}\text { Distrito Santa Catalina de Mossa } \\
\text { Caserío Caracucho }\end{array}$ & $\begin{array}{l}05^{\circ} 06^{\prime} 10^{\prime \prime S}-79^{\circ} 53^{\prime} 06^{\prime \prime} \mathrm{O} \\
05^{\circ} 14^{\prime} 02^{\prime \prime S}-79^{\circ} 91^{\prime} 56^{\prime \prime} \mathrm{O}\end{array}$ & $\begin{array}{l}\text { BSI } \\
\text { BESc }\end{array}$ & $\begin{array}{c}1500 \\
500-850\end{array}$ \\
\hline & $\begin{array}{l}\text { Caserío Ñoma } \\
\text { Bosque de Mijal }\end{array}$ & $\begin{array}{l}05^{\circ} 01^{\prime} 45^{\prime \prime} \mathrm{S}-79^{\circ} 52^{\prime} 34^{\prime \prime} \mathrm{O} \\
05^{\circ} 02^{\prime} 28^{\prime \prime} \mathrm{S}-79^{\circ} 47^{\prime} 45^{\prime \prime O}\end{array}$ & $\begin{array}{l}\mathrm{BHM} \\
\mathrm{BHM}\end{array}$ & $\begin{array}{l}2129 \\
3000\end{array}$ \\
\hline \multirow[t]{4}{*}{ Huancabamba } & Caserío Pampaminas & $05^{\circ} 21^{\prime} 20.6^{\prime \prime} \mathrm{S}-79^{\circ} 35^{\prime} 20^{\prime \prime} \mathrm{O}$ & $\mathrm{BHM}$ & 2000 \\
\hline & Cerro Huayanay & $05^{\circ} 24^{\prime} 07^{\prime \prime S}-79^{\circ} 36^{\prime} 22^{\prime \prime O}$ & $\mathrm{BHM}$ & 2000 \\
\hline & Caserío El Overal & $05^{\circ} 27^{\prime} 24^{\prime \prime} \mathrm{S}-79^{\circ} 50^{\prime} 02^{\prime \prime O}$ & BESc & 500 \\
\hline & San Miguel de El faique & $05^{\circ} 24^{\prime} 07^{\prime \prime S}-79^{\circ} 36^{\prime} 22^{\prime \prime O}$ & $\mathrm{BHM}$ & 1800 \\
\hline \multirow[t]{2}{*}{ Ayabaca } & Distrito Suyo & $04^{\circ} 30^{\prime} 45^{\prime \prime} \mathrm{S}-80^{\circ} 02^{\prime} 10^{\prime \prime} \mathrm{O}$ & BESc & 1500 \\
\hline & Caserío Yacupampa & $05^{\circ} 10^{\prime} 51^{\prime \prime} \mathrm{S}-80^{\circ} 37^{\prime} 31^{\prime \prime O}$ & BHM & $2700-3100$ \\
\hline
\end{tabular}

